\title{
THREE GENERALIZATIONS OF WEYL'S DENOMINATOR FORMULA
}

\author{
TodD Simpson \\ 7661 Tred Avon Circle, Easton, MD 21601, USA \\ todo@ora.nobis.com
}

Submitted: July 28, 1995; Accepted: March 15, 1996

\begin{abstract}
We give combinatorial proofs of three identities, each of which generalizes Weyl's denominator formula for two of the three root systems $B_{n}, C_{n}, D_{n}$. Two of the three identities are due to S. Okada; the third appears in the author's doctoral thesis, upon which this work is based.

Each of the identities we prove has a "sum side" and a "product side"; both sides are polynomials in several commuting indeterminates. We use weighted digraphs to represent the terms on each side; the set of such digraphs that corresponds to the sum side is a proper subset of the set corresponding to the product side.
\end{abstract}

"Why don't we pair 'em up in threes" — attributed to Yogi Berra

\section{Introduction}

Our purpose is to give combinatorial proofs of three identities that generalize Weyl's denominator formula. In this section, we provide some background for our work, describe how the paper is organized, and introduce some basic notation.

\section{BACKGROUND}

Weyl's denominator formula ([C], Theorem 10.1.8) is a collection of identities involving polynomials in any finite number $n$ of commuting indeterminates. There is an identity for each root system of rank $n$; a root system ([H], Chapter III) is a finite set of vectors that satisfies certain axioms. Root systems correspond to compact Lie groups and can be used to describe the characters of their representations. For details of this correspondence, see [W] or [BtD]. Weyl's character formula ([W], Kapitel IV, Satz 5; [BtD], Chapter VI, (1.7)) gives a complete description of the characters of irreducible representations of any compact, connected Lie group. The formula expresses these characters as ratios of polynomials, the denominators of which are given by Weyl's denominator formula.

1991 Mathematics Subject Classification. Primary 05A19.

Key words and phrases. Weyl's denominator formula, Schur functions, partitions, digraphs.

Partially supported by NSA Grants MDA904-90-H1010, MDA904-92-H3043 
It suffices to prove Weyl's denominator formula for irreducible root systems. A root system is irreducible if it cannot be written as a disjoint union of two nonempty, mutually orthogonal subsets. With five exceptions, every irreducible root system belongs to one of four infinite families, denoted $A, B, C$, and $D$ (cf. [H], 11.4, 12.1). The root system of rank $n$ in family $A$ is called $A_{n}$, and similarly for the other families. Gessel [G] was the first to prove Weyl's formula combinatorially for an infinite family of root systems. He gave a combinatorial proof of Vandermonde's determinant formula

$$
\sum_{\sigma \in S_{n}}(-1)^{\sigma} x_{\sigma(1)}^{n-1} x_{\sigma(2)}^{n-2} \cdots x_{\sigma(n-1)}=\operatorname{det}\left[x_{i}^{n-j}\right]_{i, j=1}^{n}=\prod_{1 \leq i<j \leq n}\left(x_{i}-x_{j}\right),
$$

where $S_{n}$ is the symmetric group on $\{1,2, \ldots, n\}$ and $(-1)^{\sigma}$ denotes the sign of the permutation $\sigma$. This identity is equivalent to Weyl's formula for $A_{n-1}$. Later, Bressoud [B] found combinatorial proofs of Weyl's formula for $B_{n}, C_{n}$, and $D_{n}$.

Each of the three identities we shall prove implies Weyl's formula for two of the three root systems $B_{n}, C_{n}, D_{n}$. Two of the identities proved here are due to Okada $[\mathrm{O}]$, though one of them is stated there without proof. The third identity appears in the author's doctoral thesis [S], upon which this work is based.

\section{ORGANIZATION}

In Section 2, we introduce the concepts and notation we need in order to state our results. The results themselves are given in Theorems 2.2 and 2.4. We also show how one of the three identities implies two cases of Weyl's formula.

We begin proving the identities in Section 3. Each of them has a "sum side" and a "product side." Our method is like that of [G] and [B], using weighted digraphs to represent the terms on each side of each identity. Section 3 introduces digraph terminology and the particular sets of digraphs and weight functions we shall use. We show that the product side of each identity can be written as a sum of weights of digraphs belonging to a particular set.

We claim that the sum side of each identity can be written as a sum of weights of digraphs belonging to a proper subset of the set that corresponds to the product side. In Section 4, we describe such a subset for each of the identities to be proved. In Section 5, we state and prove some technical lemmas to be used in the next two sections.

Sections 6 and 7 are where most of the work is done. To prove the claim above, we show in Section 6 that the digraphs not belonging to the subset described in Section 4 have weights that add to 0 . In Section 7 , we show that the digraphs that do belong to this subset have weights that add to the sum side of the identity.

Finally, there is an Appendix, which contains some discussion of the connection between the usual way of writing Weyl's denominator formula and the way we write it in Section 2. 


\section{Notational Conventions}

We write $\mathbf{N}, \mathbf{N}_{0}$, and $\mathbf{Z}$ for the sets of positive integers, nonnegative integers, and all integers; $\mathbf{R}$ denotes the set of all real numbers.

For any $n \in \mathbf{N}$, we define $[n]=\{1,2, \ldots, n\}$. If $X$ is any set, then $X^{n}$ denotes the Cartesian product of $n$ copies of $X$. If $X$ has an algebraic structure, then so does $X^{n}$; for instance, $\mathbf{R}^{n}$ is a real vector space and $\mathbf{Z}^{n}$ a free Abelian group.

We use cycle notation for permutations. For instance, $\sigma=(134)$ means that $\sigma(1)=3, \sigma(3)=4, \sigma(4)=1$, and $\sigma(i)=i$ otherwise. Permutations are composed right-to-left.

Given a statement $A$, we define $\chi(A)$ to be 1 if $A$ is true, 0 if $A$ is false.

\section{Statement of Results}

We begin this section by introducing partitions and Schur functions, which we need in order to state Theorems 2.2 and 2.4 .

\section{Partitions}

A partition is a nonincreasing sequence $\lambda=\left(\lambda_{1}, \lambda_{2}, \ldots\right)$ of nonnegative integers, with only finitely many nonzero terms. The nonzero terms of $\lambda$ are called its parts. The number of parts of a partition $\lambda$ is its length, which we denote $\ell(\lambda)$. For any $n \geq \ell(\lambda)$, we may identify $\lambda$ with the finite sequence $\left(\lambda_{1}, \lambda_{2}, \ldots, \lambda_{n}\right) \in \mathbf{N}_{0}^{n}$. Thus the expressions $(5,4,2,1),(5,4,2,1,0,0,0)$, and $(5,4,2,1,0, \ldots)$ all describe the same partition of length 4 . The weight of a partition $\lambda$, which we denote $|\lambda|$, is the sum of its parts. We say that $\lambda$ is a partition of $n$ into $k$ parts if $|\lambda|=n$ and $\ell(\lambda)=k$. For instance, $(5,4,2,1,0, \ldots)$ is a partition of 12 into 4 parts. We denote the unique partition of length and weight zero by 0 .

A useful idea in the study of partitions is that of the Ferrers diagram of a partition $\lambda$. This is the set $D(\lambda)=\left\{(i, j) \in \mathbf{N}^{2}: 1 \leq i \leq \ell(\lambda), 1 \leq j \leq \lambda_{i}\right\}$. One often thinks of $D(\lambda)$ as a left-justified array of unit squares, in which the number of squares in the $i$ th highest row is $\lambda_{i}$. Figure 2.1 portrays the Ferrers diagram of $(5,4,2,1)$.

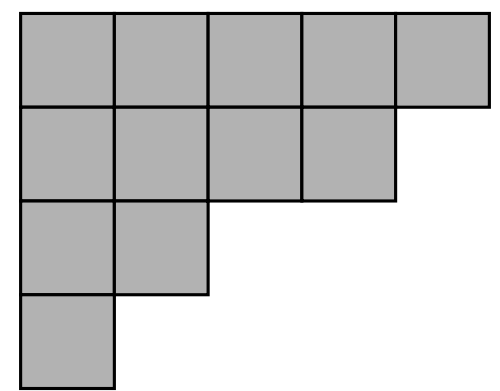

FIGURE 2.1 
The rank of a partition $\lambda$, denoted $p(\lambda)$, is the largest $i$ such that $(i, i) \in D(\lambda)$. Equivalently, $p(\lambda)=\max \left\{i: \lambda_{i} \geq i\right\}$. For example, the partition $(5,4,2,1)$ has rank 2.

If $\lambda$ is a partition, then the set $\{(j, i):(i, j) \in D(\lambda)\}$ is the Ferrers diagram of a partition $\lambda^{\prime}$, called the conjugate of $\lambda$. For example, the conjugate of $(5,4,2,1)$ is $(4,3,2,2,1)$. We have $\ell\left(\lambda^{\prime}\right)=\lambda_{1},\left|\lambda^{\prime}\right|=|\lambda|, p\left(\lambda^{\prime}\right)=p(\lambda)$, and $\lambda^{\prime \prime}=\lambda$ for any partition $\lambda$. We say that $\lambda$ is self-conjugate if $\lambda=\lambda^{\prime}$. An alternative definition of $\lambda^{\prime}$, which does not require Ferrers diagrams, is given by $\lambda_{i}^{\prime}=\max \left\{j: \lambda_{j} \geq i\right\}$.

We now define the Frobenius representation of a partition $\lambda$. For each $i \in[p(\lambda)]$, let $\alpha_{i}=\lambda_{i}-i$ and $\beta_{i}=\lambda_{i}^{\prime}-i$. Both $\left(\alpha_{1}, \ldots, \alpha_{p(\lambda)}\right)$ and $\left(\beta_{1}, \ldots, \beta_{p(\lambda)}\right)$ are strictly decreasing sequences of nonnegative integers. The Frobenius representation of $\lambda$ is $\left(\alpha_{1}, \ldots, \alpha_{p(\lambda)} \mid \beta_{1}, \ldots, \beta_{p(\lambda)}\right)$, or more concisely $(\alpha \mid \beta)$. We note that $|\lambda|=$ $p(\lambda)+\sum_{i=1}^{p(\lambda)}\left(\alpha_{i}+\beta_{i}\right)$. And if the Frobenius representation of $\lambda$ is $(\alpha \mid \beta)$, then $\lambda^{\prime}=(\beta \mid \alpha)$.

If $\lambda$ and $\mu$ are partitions, then $D(\mu) \subseteq D(\lambda)$ if and only if $\mu_{i} \leq \lambda_{i}$ for all $i \geq 1$. It is customary to identify partitions with their Ferrers diagrams, so one usually writes $\mu \subseteq \lambda$ instead of $D(\mu) \subseteq D(\lambda)$. The relation $\subseteq$ is a partial order on the set of all partitions. In case $\mu \subseteq \lambda$, we define the skew diagram $\lambda-\mu$; this is simply the set-theoretic difference $D(\lambda) \backslash D(\mu)$. One may think of it as an array of unit squares in which the $i$ th highest row is indented $\mu_{i}$ units and contains $\lambda_{i}-\mu_{i}$ squares. For example, Figure 2.2 depicts the skew diagrams $(5,3,3,1)-(3,2,2,1)$ and $(4,2,2,1)-(3,2,1)$.
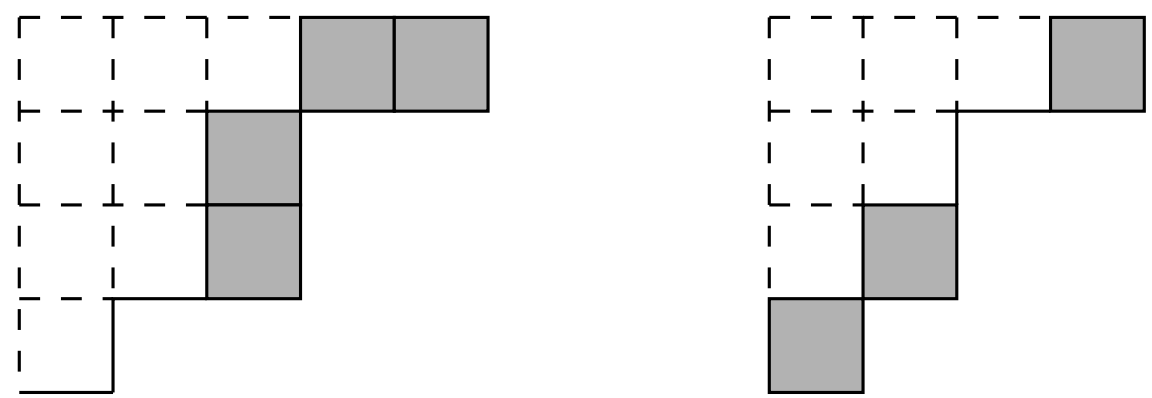

Figure 2.2

Observe that no row of $(4,2,2,1)-(3,2,1)$ contains more than one square. A skew diagram with this property is called a vertical strip. Evidently $\lambda-\mu$ is a vertical strip if $0 \leq \lambda_{i}-\mu_{i} \leq 1$ for all $i$.

\section{SCHUR FUnCTIONS}

Let $x_{1}, x_{2}, \ldots, x_{n}$ be commuting indeterminates and let $\alpha=\left(\alpha_{1}, \ldots, \alpha_{n}\right) \in$ $\mathbf{N}_{0}^{n}$. We denote by $x^{\alpha}$ the monomial $x_{1}^{\alpha_{1}} x_{2}^{\alpha_{2}} \cdots x_{n}^{\alpha_{n}}$. The ring $\mathbf{Z}\left[x_{1}, \ldots, x_{n}\right]$ of polynomials in $x_{1}, \ldots, x_{n}$ with integer coefficients is generated as a free $\mathbf{Z}$-module by the monomials.

The symmetric group $S_{n}$ acts on $\mathbf{Z}\left[x_{1}, \ldots, x_{n}\right]$ by permuting indeterminates. We define $x^{\sigma(\alpha)}=x_{\sigma(1)}^{\alpha_{1}} x_{\sigma(2)}^{\alpha_{2}} \cdots x_{\sigma(n)}^{\alpha_{n}}$ for any $\sigma \in S_{n}$ and $\alpha \in \mathbf{N}_{0}^{n}$. A polynomial 
$f=\sum_{\alpha} f_{\alpha} x^{\alpha} \in \mathbf{Z}\left[x_{1}, \ldots, x_{n}\right]$ is said to be symmetric if it is invariant under this action of $S_{n}$, in other words, if

$$
f\left(x_{1}, \ldots, x_{n}\right)=\sum_{\alpha} f_{\alpha} x^{\alpha}=\sum_{\alpha} f_{\alpha} x^{\sigma(\alpha)}=f\left(x_{\sigma(1)}, \ldots, x_{\sigma(n)}\right)
$$

for all $\sigma \in S_{n}$. A polynomial $a \in \mathbf{Z}\left[x_{1}, \ldots, x_{n}\right]$ such that

$$
a\left(x_{1}, \ldots, x_{n}\right)=(-1)^{\sigma} a\left(x_{\sigma(1)}, \ldots, x_{\sigma(n)}\right)
$$

for all $\sigma \in S_{n}$ is called antisymmetric. If $a$ is antisymmetric, then $a=0$ whenever $x_{i}=x_{j}$ for some $i \neq j$. This implies that $a$ is divisible by

$$
\Delta_{n}=\prod_{1 \leq i<j \leq n}\left(x_{i}-x_{j}\right)
$$

And since $\Delta_{n}$ is itself antisymmetric, the quotient $a / \Delta_{n}$ is symmetric.

Let $\lambda$ be a partition of length at most $n$ and let $\delta=\delta_{n}=(n-1, \ldots, 1,0)$. The polynomial

$$
a_{\lambda+\delta}\left(x_{1}, \ldots, x_{n}\right)=\sum_{\sigma \in S_{n}}(-1)^{\sigma} x^{\sigma(\lambda+\delta)}=\operatorname{det}\left[x_{i}^{\lambda_{j}+n-j}\right]_{i, j=1}^{n}
$$

is antisymmetric, and therefore

$$
s_{\lambda}\left(x_{1}, \ldots, x_{n}\right)=\frac{a_{\lambda+\delta}\left(x_{1}, \ldots, x_{n}\right)}{\Delta_{n}\left(x_{1}, \ldots, x_{n}\right)}=\frac{a_{\lambda+\delta}}{a_{\delta}}
$$

is a symmetric polynomial, called the Schur function corresponding to $\lambda$. Note that Vandermonde's determinant formula implies the equality of the two denominators in (2.1).

\section{LitTLEWOOD'S IDENTITIES}

In his study of the characters of representations of orthogonal groups, Littlewood derived several identities involving Schur functions ([L], p. 238). Our results are generalizations of three of these identities.

For any integer $t$, let $P_{t}(n)$ denote the set of all partitions

$$
\lambda=\left(\alpha_{1}+t, \alpha_{2}+t, \ldots, \alpha_{p}+t \mid \alpha_{1}, \alpha_{2}, \ldots, \alpha_{p}\right)
$$

such that $\ell(\lambda) \leq n$. For instance, $(5,4,2,1) \in P_{1}(n)$ for all $n \geq 4$. We write $P_{t}$ to denote the set of all partitions belonging to $P_{t}(n)$ for sufficiently large $n$. If $t$ is odd, then the partitions in $P_{t}$ all have even weight; if $t$ is even, then $|\lambda|+p(\lambda)$ is even for all $\lambda \in P_{t}$. 
We now state the three of Littlewood's Schur function identities that our results generalize:

$(2.2 \mathrm{~b})$

$$
\prod_{i=1}^{n}\left(1-x_{i}\right) \prod_{1 \leq i<j \leq n}\left(1-x_{i} x_{j}\right)=\sum_{\lambda \in P_{0}(n)}(-1)^{(|\lambda|+p(\lambda)) / 2} s_{\lambda}\left(x_{1}, \ldots, x_{n}\right) ;
$$

$$
\begin{aligned}
\prod_{i=1}^{n}\left(1-x_{i}^{2}\right) \prod_{1 \leq i<j \leq n}\left(1-x_{i} x_{j}\right) & =\sum_{\lambda \in P_{1}(n)}(-1)^{|\lambda| / 2} s_{\lambda}\left(x_{1}, \ldots, x_{n}\right) \\
\prod_{1 \leq i<j \leq n}\left(1-x_{i} x_{j}\right) & =\sum_{\lambda \in P_{-1}(n)}(-1)^{|\lambda| / 2} s_{\lambda}\left(x_{1}, \ldots, x_{n}\right) .
\end{aligned}
$$

Multiply these by Vandermonde's determinant to obtain the equivalent identities

$$
\prod_{i=1}^{n}\left(1-x_{i}\right) \prod_{1 \leq i<j \leq n}\left(1-x_{i} x_{j}\right)\left(x_{i}-x_{j}\right)=\sum_{\substack{\lambda \in P_{0}(n) \\ \sigma \in S_{n}}}(-1)^{(|\lambda|+p(\lambda) / 2)}(-1)^{\sigma} x^{\sigma\left(\lambda+\delta_{n}\right)}
$$

$$
\begin{aligned}
& \prod_{i=1}^{n}\left(1-x_{i}^{2}\right) \prod_{1 \leq i<j \leq n}\left(1-x_{i} x_{j}\right)\left(x_{i}-x_{j}\right)=\sum_{\substack{\lambda \in P_{1}(n) \\
\sigma \in S_{n}}}(-1)^{|\lambda| / 2}(-1)^{\sigma} x^{\sigma\left(\lambda+\delta_{n}\right)} \\
&2.3 \mathrm{~d}) \quad \prod_{1 \leq i<j \leq n}\left(1-x_{i} x_{j}\right)\left(x_{i}-x_{j}\right)=\sum_{\substack{\lambda \in P_{-1}(n) \\
\sigma \in S_{n}}}(-1)^{|\lambda| / 2}(-1)^{\sigma} x^{\sigma\left(\lambda+\delta_{n}\right)}
\end{aligned}
$$

We think of the latter identities as cases of Weyl's denominator formula. Macdonald ([M], p. 46) observed that Weyl's formula for the root system $B_{n}$ (respectively, $C_{n}, D_{n}$ ) implies (2.2b) (respectively, (2.2c), (2.2d)). Bressoud's [B] combinatorial proofs of Weyl's formula for $B_{n}, C_{n}$, and $D_{n}$ are in fact proofs of (2.3b), (2.3c), and (2.3d). See the Appendix for details on the relation between Littlewood's identities and Weyl's formula.

\section{Generalizations}

We begin by defining the sets of partitions that will index the terms on the "sum sides" of our generalizations of Weyl's formula:

$P_{-1,0}(n)$ consists of all $\lambda=\left(\alpha_{1}, \ldots, \alpha_{p} \mid \beta_{1}, \ldots, \beta_{p}\right)$ such that

$$
n-1 \geq \beta_{1} \geq \alpha_{1} \geq \beta_{2} \geq \alpha_{2} \geq \cdots \geq \beta_{p} \geq \alpha_{p} .
$$

$P_{0,1}(n)$ consists of all $\lambda=\left(\alpha_{1}, \ldots, \alpha_{p} \mid \beta_{1}, \ldots, \beta_{p}\right)$ such that

$$
n \geq \beta_{1}+1 \geq \alpha_{1} \geq \beta_{2}+1 \geq \alpha_{2} \geq \cdots \geq \beta_{p}+1 \geq \alpha_{p} .
$$

$P_{-1,1}(n)$ is the set of all $\lambda$ with $\ell(\lambda) \leq n$ such that for some $\kappa \in P_{-1}(n)$, we have $0 \leq \lambda_{i}-\kappa_{i} \leq 2$ for all $i \in[n]$ and $\left\{i \in[n]: \lambda_{i}-\kappa_{i}=1\right\}$ is a disjoint union of pairs $\{j, j+1\}$ with $\kappa_{j}=\kappa_{j+1}$. 
An alternative definition of $P_{-1,1}(n)$ may be given in terms of Ferrers diagrams. A domino is a subset of $\mathbf{Z}^{2}$ of the form $\{(i, j),(i, j+1)\}$ or $\{(i, j),(i+1, j)\}$. Just as we visualize Ferrers diagrams as arrays of unit squares, we think of dominos as pairs of squares having a common edge. A partition $\lambda$ of length at most $n$ is in $P_{-1,1}(n)$ if and only if $\lambda-\kappa$ can be written as a disjoint union of dominos, with at most one domino per row, for some $\kappa \in P_{-1}(n)$. In Figure 2.3, we have two partitions $\lambda$ such that $\lambda-\kappa$ is a disjoint union of dominos for $\kappa=(3,2,2,1) \in P_{-1}(4)$. The diagram on the left shows that $(5,3,3,1)$ is in $P_{-1,1}(n)$ for any $n \geq 4$, because no row has more than one domino. This condition is violated on the right, as indeed it must be, since the partition $(4,4,3,1)$ is not in $P_{-1,1}(4)$.
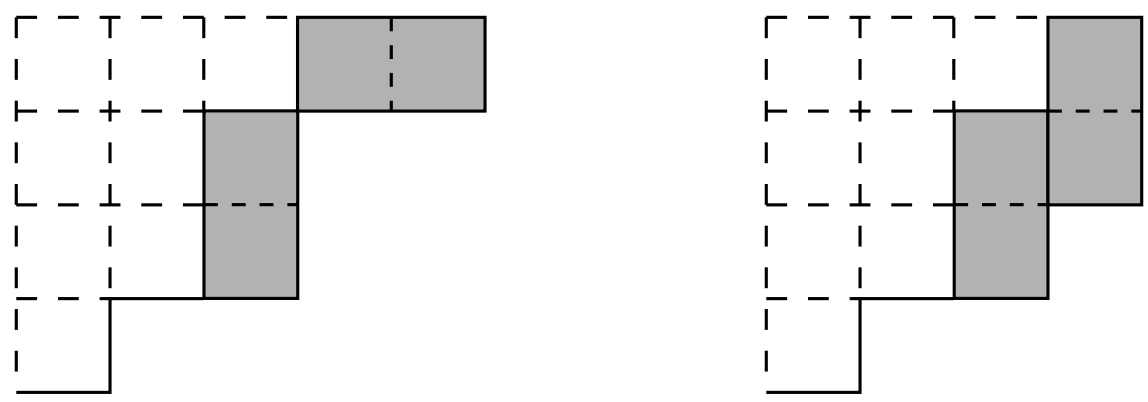

FiguRe 2.3

We have $P_{i}(n) \cup P_{j}(n) \subset P_{i, j}(n)$ for $-1 \leq i<j \leq 1$. We also note that $|\lambda|$ is even whenever $\lambda \in P_{-1,1}(n)$.

To state the first of our three generalizations, we shall need to describe, for any partition $\lambda \in P_{-1,0}(n)$, the largest $\mu \in P_{-1}(n)$ such that $\mu \subseteq \lambda$. To state the second generalization, we shall need, given $\lambda \in P_{0,1}(n)$, the largest $\nu \in P_{0}(n)$ such that $\nu \subseteq \lambda$. The partitions $\mu$ and $\nu$ may be described in terms of their Ferrers diagrams as follows.

For any partition $\lambda$, we define the following subsets of $\mathbf{N}^{2}$ :

$$
\begin{aligned}
M(\lambda) & =\left\{(i, j-1): i<j, \lambda_{i}+\lambda_{j} \geq i+j-1\right\} ; \\
M^{\prime}(\lambda) & =\{(j, i):(i, j-1) \in M(\lambda)\} ; \\
N(\lambda) & =\left\{(i, j): \lambda_{i}+\lambda_{j} \geq i+j\right\} .
\end{aligned}
$$

The sets $M(\lambda) \cup M^{\prime}(\lambda)$ and $N(\lambda)$ are Ferrers diagrams of partitions. Let $\mu=\mu(\lambda)$ and $\nu=\nu(\lambda)$ denote these partitions, so that $D(\mu)=M(\lambda) \cup M^{\prime}(\lambda)$ and $D(\nu)=$ $N(\lambda)$. There is some $p \geq 0$ and $\alpha_{1}>\alpha_{2}>\cdots>\alpha_{p} \geq 1$ such that

$$
D(\mu)=\bigcup_{i=1}^{p}\left\{(i, i), \ldots,\left(i, i+\alpha_{i}-1\right),(i+1, i), \ldots,\left(i+\alpha_{i}, i\right)\right\} .
$$

This shows that $\mu(\lambda) \in P_{-1}(n)$ for all sufficiently large $n$. Meanwhile, we have $(i, j) \in N(\lambda)$ if and only if $(j, i) \in N(\lambda)$, which implies that $\nu(\lambda)$ is self-conjugate, or equivalently that $\nu(\lambda) \in P_{0}(n)$ for large enough $n$. The table in Figure 2.4 compares $\lambda_{i}+\lambda_{j}$ to $i+j-1$, where $\lambda=(5,4,4,3,2)$. The diagram depicts $D(\mu)$, the bold line separating $M(\lambda)$ from $M^{\prime}(\lambda)$. 


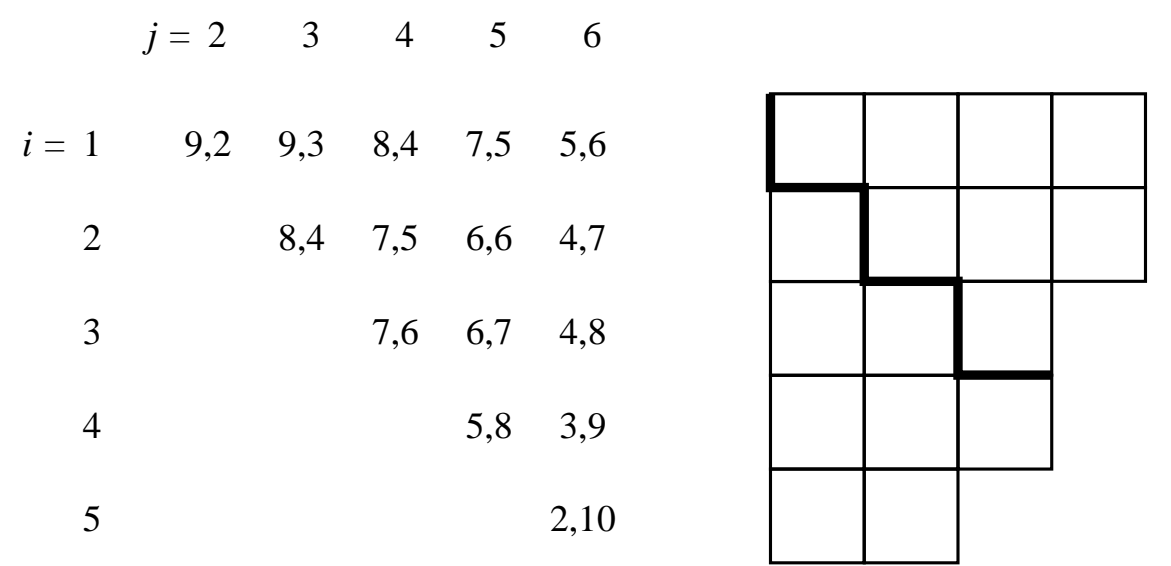

Figure 2.4

The following lemma tells us that $\mu$ and $\nu$ have the properties we need. We shall prove it in Section 7 .

Lemma 2.1. ([S], Lemma 3.1.1) (a) If $\lambda \in P_{-1,0}(n)$, then $\mu(\lambda) \subseteq \lambda$, and we have $\xi \subseteq \mu(\lambda)$ for any $\xi \in P_{-1}(n)$ such that $\xi \subseteq \lambda$.

(b) If $\lambda \in P_{0,1}(n)$, then $\nu(\lambda) \subseteq \lambda$, and we have $\xi \subseteq \nu(\lambda)$ for any $\xi \in P_{0}(n)$ such that $\xi \subseteq \lambda$.

Given any partition $\lambda$, we define

and

$$
y(\lambda)=\left|\left\{(i, j): i<j, \lambda_{i}+\lambda_{j}=i+j-1\right\}\right|
$$

$$
z(\lambda)=\left|\left\{(i, j): i<j, \lambda_{i}+\lambda_{j}=i+j\right\}\right|
$$

We are ready to state the first two of our three generalizations of Weyl's formula:

Theorem 2.2. ([S], Theorem 3.1.2) We have

$$
\begin{gathered}
\prod_{1 \leq i \leq n}\left(1-t x_{i}\right) \prod_{1 \leq i<j \leq n}\left(1-x_{i} x_{j}\right)\left(x_{i}-x_{j}\right) \\
=\sum_{\substack{\lambda \in P_{-1,0}(n) \\
\sigma \in S_{n}}}(-1)^{|\lambda|-|\mu| / 2} t^{|\lambda|-|\mu|}\left(1-t^{2}\right)^{y(\lambda)}(-1)^{\sigma} x^{\sigma\left(\lambda+\delta_{n}\right)}
\end{gathered}
$$

and

$(\mathrm{BC})$

$$
\prod_{1 \leq i \leq n}\left(1-x_{i}\right)\left(1+t x_{i}\right) \prod_{1 \leq i<j \leq n}\left(1-x_{i} x_{j}\right)\left(x_{i}-x_{j}\right)
$$

$=\sum_{\substack{\lambda \in P_{0,1}(n) \\ \sigma \in S_{n}}}(-1)^{(|\nu|+p(\nu)) / 2} t^{|\lambda|-|\nu|}(1-t)^{\chi\left(\exists i, \lambda_{i}=i\right)}\left(1-t^{2}\right)^{z(\lambda)}(-1)^{\sigma} x^{\sigma\left(\lambda+\delta_{n}\right)}$ 
for all $n \geq 1$.

In [O], Okada proves an identity (Lemma 3.5) equivalent to (BC). He also states without proof an identity equivalent to (BD).

At the end of this section, we shall show how (BD) specializes to (2.3d) at $t=0$ and to $(2.3 \mathrm{~b})$ at $t=1$. A similar argument shows that $(\mathrm{BC})$ gives us $(2.3 \mathrm{~b})$ and $(2.3 \mathrm{c})$ at $t=0$ and $t=1$ respectively.

In order to state the third of our generalizations of Weyl's formula, we shall need, given $\lambda \in P_{-1,1}(n)$, the largest $\kappa \in P_{-1}(n)$ such that $\kappa \subseteq \lambda$ and $\lambda-\kappa$ is a disjoint union of dominos with no more than one domino per row. We have not found an elegant description of this partition $\kappa$ like those of $\mu$ and $\nu$ above.

Given a partition $\lambda$, let $K(\lambda)$ be the set of all partitions $\eta \subseteq \lambda$ such that: $\eta \in P_{-1}(n)$ for some $n$, and $\lambda-\eta$ is a disjoint union of dominos with no more than one domino in any one row of $D(\lambda)$. Observe that $\lambda \in P_{-1,1}(n)$ for some $n$ if and only if $K(\lambda) \neq \emptyset$.

Figure 2.5 shows that $\lambda=(6,5,5,3,3,2)$ is in $P_{-1,1}(n)$ for $n \geq 6$. The partitions $(3,2,1 \mid 4,3,2)$ and $(3,1,0 \mid 4,2,1)$ belong to $K(\lambda)$. On the other hand, $\eta=(3,2,0 \mid 4,3,1)$ is not in $K(\lambda)$, even though $0 \leq \lambda_{i}-\eta_{i} \leq 2$ for all $i$, because $\lambda-\eta$ cannot be written as a disjoint union of dominos.

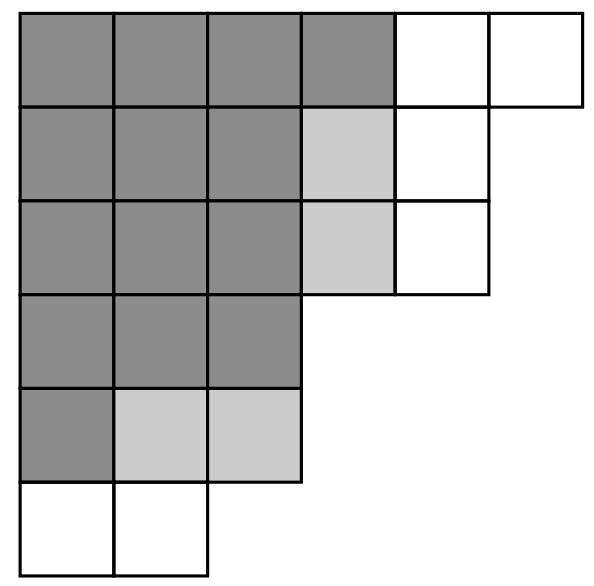

FiguRe 2.5

Lemma 2.3 tells us that there is a largest partition in $K(\lambda)$ for any $\lambda \in P_{-1,1}(n)$. We shall prove it in Section 7 .

Lemma 2.3. ([S], Lemma 3.1.3) For any $\lambda \in P_{-1,1}(n)$, there is a partition $\kappa=$ $\kappa(\lambda) \in K(\lambda)$ such that $\eta \subseteq \kappa$ for all $\eta \in K(\lambda)$.

The role $\kappa$ will play in our third generalization is like that of $\mu$ in (BD) and of $\nu$ in (BC). We remark that $\kappa$ is defined only for partitions in $P_{-1,1}(n)$, whereas the definitions of $\mu$ and $\nu$ make sense for any partition.

Given $\lambda \in P_{-1,1}(n)$, let $q(\lambda)$ be the number of "horizontal" dominos in $\lambda-\kappa$. Equivalently, $q(\lambda)$ is the cardinality of the set $\left\{i \in[n]: \lambda_{i}-\kappa_{i}=2\right\}$. For instance, 
with $\lambda$ as in Figure 2.5, we have $\kappa=(3,2,1 \mid 4,3,2)$ and $q(\lambda)=2$. We can now state our third generalization of Weyl's formula:

Theorem 2.4. ([S], Theorem 3.1.4) We have

$$
\begin{gathered}
\prod_{1 \leq i \leq n}\left(1-t x_{i}^{2}\right) \prod_{1 \leq i<j \leq n}\left(1-x_{i} x_{j}\right)\left(x_{i}-x_{j}\right) \\
=\sum_{\substack{\lambda \in P_{-1,1}(n) \\
\sigma \in S_{n}}}(-1)^{|\kappa| / 2+q(\lambda)} t^{(|\lambda|-|\kappa|) / 2}(1-t)^{\chi\left(\exists i, \lambda_{i}=i\right)}\left(1-t^{2}\right)^{z(\lambda)}(-1)^{\sigma} x^{\sigma\left(\lambda+\delta_{n}\right)}
\end{gathered}
$$

for any $n \geq 1$.

We obtain (2.3d) from (CD) by setting $t=0$ and $(2.3 \mathrm{c})$ by setting $t=1$.

$$
\text { Obtaining (2.3d) AND (2.3b) FRom (BD) }
$$

If $t=0$, then the product side of (BD) is the same as that of $(2.3 \mathrm{~d})$. The sum side of (BD), meanwhile, is

$$
\sum_{\substack{\lambda \in P-1,0(n):|\lambda|=|\mu| \\ \sigma \in S_{n}}}(-1)^{|\lambda| / 2}(-1)^{\sigma} x^{\sigma\left(\lambda+\delta_{n}\right)} .
$$

Lemma 2.1(a) tells us that $\mu \subseteq \lambda$ for any $\lambda \in P_{-1,0}(n)$. If $\mu \subseteq \lambda$ and $|\lambda|=|\mu|$, then clearly $\lambda=\mu$. Therefore the latter sum is taken over $P_{-1}(n)$; it is the sum side of $(2.3 \mathrm{~d})$.

Now suppose $t=1$. The product sides of $(\mathrm{BD})$ and $(2.3 \mathrm{~b})$ coincide, whereas the sum side of (BD) is

$$
\sum_{\substack{\lambda \in P_{-1,0}(n): y(\lambda)=0 \\ \sigma \in S_{n}}}(-1)^{|\lambda|-|\mu| / 2}(-1)^{\sigma} x^{\sigma\left(\lambda+\delta_{n}\right)} .
$$

If $\lambda \in P_{-1,0}(n)$ and $y(\lambda)=0$, then the quantities $\left|\lambda_{i}-i+\frac{1}{2}\right|, i \in[n]$, are distinct. And they are not larger than $n-\frac{1}{2}$, since $\lambda_{1} \leq n$. In this situation, Lemma A.1 (in the Appendix) implies that $\lambda \in P_{0}(n)$, and it remains only to show that $|\lambda|-\frac{|\mu|}{2}=\frac{|\lambda|+p(\lambda)}{2}$, or equivalently, $|\lambda|-|\mu|=p(\lambda)$. To this end, let $p=p(\lambda)$ and $q=p-\chi\left(\lambda_{p}=p\right)$. Then for $1 \leq i \leq q$ and $i<j \leq \lambda_{i}$, we have $(i, j) \in D(\lambda)$, and since $\lambda=\lambda^{\prime}$, we have $(j, i) \in D(\lambda)$. Therefore $\lambda_{i} \geq j, \lambda_{j} \geq i, \lambda_{i}+\lambda_{j}>i+j-1$, and we have $(i, j-1),(j, i) \in D(\mu)$. Meanwhile, for $1 \leq i \leq p$ and $j \geq \lambda_{i}+1$, we have $\lambda_{i}+\lambda_{j}<i+j-1$. This means that if $\lambda=\left(\alpha_{1}, \ldots, \alpha_{p} \mid \alpha_{1}, \ldots, \alpha_{p}\right)$, then $\mu=\left(\alpha_{1}-1, \ldots, \alpha_{q}-1 \mid \alpha_{1}, \ldots, \alpha_{q}\right)$. We conclude that $|\lambda|-|\mu|=p$ as required. 


\section{The Product Sides}

We shall now introduce the digraphs and weight functions to be used in proving (BD), (BC), and (CD).

Let $V$ be a set, whose elements we call vertices. We shall say that a subset $\mathfrak{g}$ of $V \times V$ is a digraph on $V$ if it has the following two properties:

For all $i \in V,(i, i) \notin \mathfrak{g}$.

If $(i, j) \in \mathfrak{g}$, then $(j, i) \notin \mathfrak{g}$.

We call the elements of $\mathfrak{g}$ arcs; we say that $(i, j)$ is an arc from $i$ to $j$. Observe that by our definition, a digraph cannot have an arc from a vertex to itself, nor can it have more than one arc between two distinct vertices. We say that $\mathfrak{g}$ is complete if it has an arc between any two distinct vertices. In other words, $\mathfrak{g}$ is complete if we have either $(i, j) \in \mathfrak{g}$ or $(j, i) \in \mathfrak{g}$ whenever $i \neq j$. Complete digraphs on finite sets of vertices are often called tournaments.

We shall work exclusively with digraphs on finite vertex sets. Such digraphs can be visualized, using points in the plane to represent vertices and arrows from one point to another to represent arcs. In Figure $3.1, \mathfrak{g}_{1}=\{(a, d),(b, a),(b, d),(e, c)\}$ and $\mathfrak{g}_{2}=\{(1,2),(1,3),(2,3),(3,4),(4,1),(4,2)\}$ are digraphs on $\{a, b, c, d, e\}$ and [4] respectively.
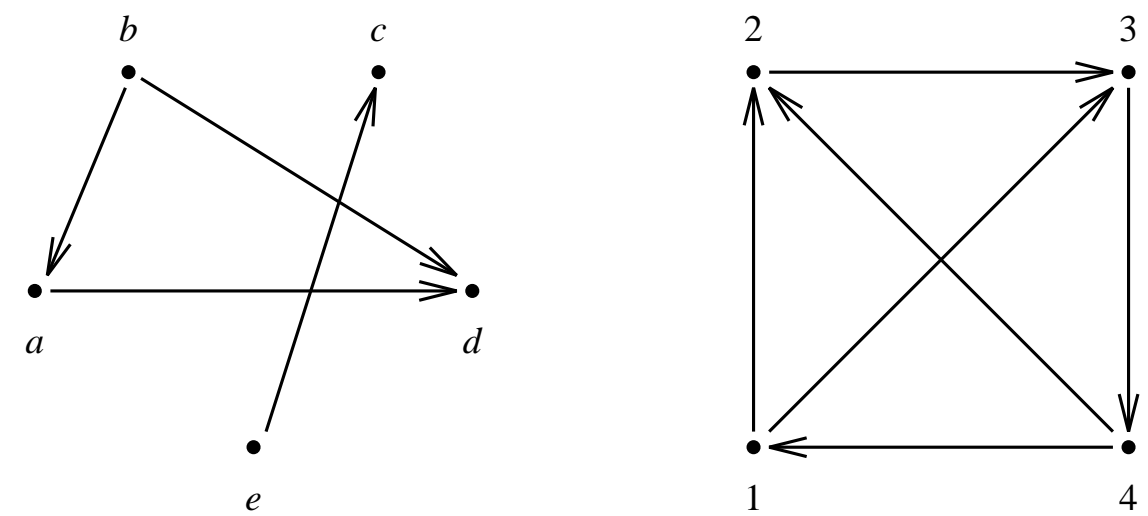

Figure 3.1

The out-degree of a vertex $i$ in a digraph $\mathfrak{g}$ is the number of arcs in $\mathfrak{g}$ from $i$ to other vertices. We denote this number by $o(i, \mathfrak{g})$. In Figure 3.1, for example, $o\left(b, \mathfrak{g}_{1}\right)=2$ and $o\left(3, \mathfrak{g}_{2}\right)=1$. A path from $i$ to $j$ is a sequence of arcs $\left(i, k_{1}\right)$, $\left(k_{1}, k_{2}\right), \ldots,\left(k_{n-1}, j\right)$ in $\mathfrak{g}$. The length of a path is the number of arcs it contains. A cycle is a path from a vertex to itself. By our definition of digraphs, any cycle must have length at least 3 . We say $\mathfrak{g}$ is transitive if it contains no cycles. For instance, $\mathfrak{g}_{1}$ is transitive, but $\mathfrak{g}_{2}$ has a cycle. If $\mathfrak{g}$ is a digraph on $V$ and $W \subseteq V$, then $\mathfrak{g} \cap(W \times W)$ is a digraph on $W$, the restriction of $\mathfrak{g}$ to $W$. For example, the restriction of $\mathfrak{g}_{2}$ to $[3]$ is $\{(1,2),(1,3),(2,3)\}$.

We are ready to define the sets of digraphs we shall use in our proofs of Theorems 2.2 and 2.4. Given a positive integer $n$, let $V_{n}=\{-n, \ldots,-1,0,1, \ldots, n\}$. Define sets $\mathfrak{C}_{n}$ and $\mathfrak{B}_{n}$ of digraphs on $V_{n}$ as follows: 
$\mathfrak{C}_{n}$ consists of all complete digraphs $\mathfrak{c}$ on $V_{n}$ such that $(-j,-i) \in \mathfrak{c}$ whenever $(i, j) \in \mathfrak{c}$.

$\mathfrak{B}_{n}$ consists of all digraphs $\mathfrak{b}$ on $V_{n}$ such that: for all $i \in V_{n},(i,-i) \notin \mathfrak{b}$; for all $i, j \in V_{n}$ with $j \neq \pm i$, either $(i, j) \in \mathfrak{b}$ or $(j, i) \in \mathfrak{b}$; and $(-j,-i) \in \mathfrak{b}$ whenever $(i, j) \in \mathfrak{b}$. (Given $\mathfrak{c} \in \mathfrak{C}_{n}$, the digraph $\mathfrak{b}$ we obtain from $\mathfrak{c}$ by deleting all arcs of the form $(i,-i)$ is in $\mathfrak{B}_{n}$.)

We shall use $\mathfrak{B}_{n}$ in our proof of (BD) and $\mathfrak{C}_{n}$ in our proofs of (BC) and (CD). Observe that for any $\mathfrak{g} \in \mathfrak{B}_{n} \cup \mathfrak{C}_{n}$, the restriction of $\mathfrak{g}$ to $[n]$ is a complete digraph, which we call the positive subtournament of $\mathfrak{g}$ and denote by $\mathfrak{g}^{+}$.

Our proofs begin with the assignment of a weight to each digraph in the appropriate set. Given a digraph $\mathfrak{g}$, we assign a weight $w(i, j)$ to each $\operatorname{arc}(i, j)$ in $\mathfrak{g}$. The product of all these weights is the weight of $\mathfrak{g}: w(\mathfrak{g})=\prod_{(i, j) \in \mathfrak{g}} w(i, j)$. We use the following functions to assign weights to arcs:

$$
\begin{aligned}
& \hat{w}(i, j)=(-1)^{\chi(i>|j|)}\left(x_{i} t^{\chi(j=0)}\right)^{\chi(i>0)} \\
& \tilde{w}(i, j)=(-1)^{\chi(i>|j|)}\left(x_{i} \chi^{\chi(j=-i)}\right)^{\chi(i>0)} \\
& \breve{w}(i, j)=(-1)^{\chi(i>|j|)}\left(x_{i} t^{\chi(j=0 \text { or } j=-i) / 2}\right)^{\chi(i>0)} .
\end{aligned}
$$

We remark that if $p \leq 0$, then $\hat{w}(p, q)=\tilde{w}(p, q)=\breve{w}(p, q)=1$ for all $q$, and that if $p$ and $q$ are nonzero and $q \neq-p$, then $\hat{w}(p, q)=\tilde{w}(p, q)=\breve{w}(p, q)$. For example, if $\mathfrak{g}$ is the digraph in $\mathfrak{B}_{3}$ shown in Figure 3.2 , then $\hat{w}(\mathfrak{g})=t x_{1}^{4} x_{2} x_{3}^{3}$.

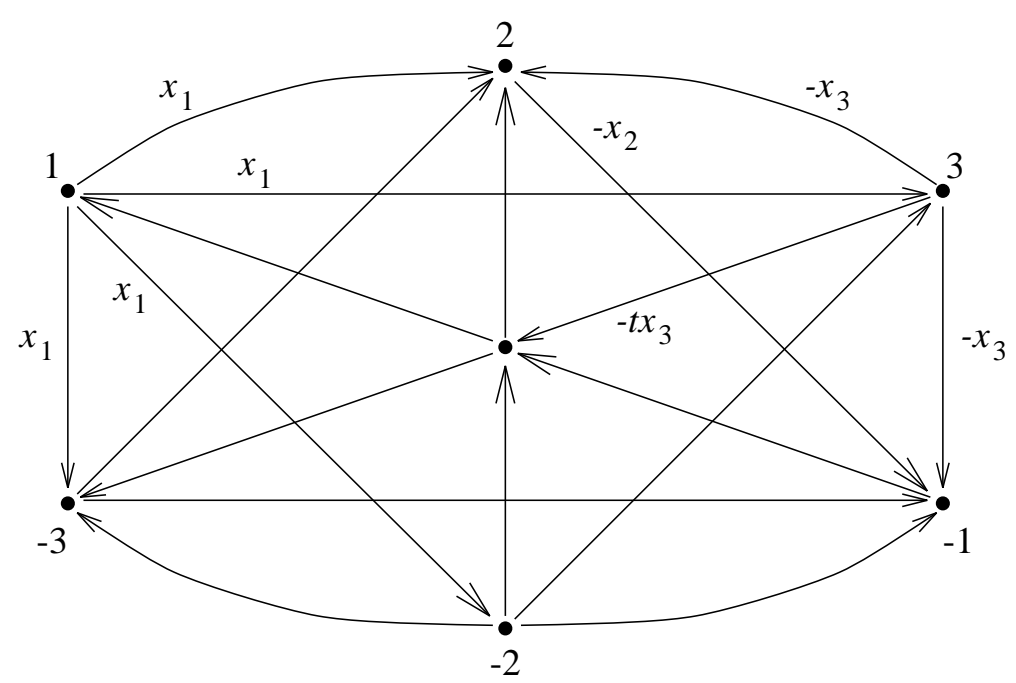

FiguRE 3.2 
We can now write the product sides of (BD), (BC), and (CD) as sums indexed by digraphs:

$$
\prod_{1 \leq i \leq n}\left(1-t x_{i}\right) \prod_{1 \leq i<j \leq n}\left(1-x_{i} x_{j}\right)\left(x_{i}-x_{j}\right)=\sum_{\mathfrak{b} \in \mathfrak{B}_{n}} \hat{w}(\mathfrak{b})
$$

$$
\begin{aligned}
\prod_{1 \leq i \leq n}\left(1-x_{i}\right)\left(1+t x_{i}\right) \prod_{1 \leq i<j \leq n}\left(1-x_{i} x_{j}\right)\left(x_{i}-x_{j}\right) & =\sum_{\mathfrak{c} \in \mathfrak{C}_{n}} \tilde{w}(\mathfrak{c}), \\
\prod_{1 \leq i \leq n}\left(1-t x_{i}^{2}\right) \prod_{1 \leq i<j \leq n}\left(1-x_{i} x_{j}\right)\left(x_{i}-x_{j}\right) & =\sum_{\mathfrak{c} \in \mathfrak{C}_{n}} \check{w}(\mathfrak{c}) .
\end{aligned}
$$

To prove $(3.1 \mathrm{~cd})$, consider a digraph $\mathfrak{c} \in \mathfrak{C}_{n}$. For each pair $i, j$ of integers with $1 \leq i<j \leq n$, we have either $(i, j)$ and $(-j,-i)$ in $\mathfrak{c}$, contributing $x_{i}$ and 1 to $\breve{w}(\mathfrak{c})$, or we have $(j, i)$ and $(-i,-j)$ in $\mathfrak{c}$, contributing $-x_{j}$ and 1 . We have either $(i,-j),(j,-i) \in \mathfrak{c}$, contributing $-x_{i} x_{j}$ to $\breve{w}(\mathfrak{c})$, or $(-i, j),(-j, i) \in \mathfrak{c}$, contributing 1. For each $i$ with $1 \leq i \leq n$, we have either $(i,-i)$ or $(-i, i)$, contributing either $t^{1 / 2} x_{i}$ or 1 , and either $(i, 0),(0,-i)$ or $(0, i),(-i, 0)$, contributing either $-t^{1 / 2} x_{i}$ or 1. This accounts for all the arcs of $\mathfrak{c}$. We conclude that the sum of $\breve{w}(\mathfrak{c})$ as $\mathfrak{c}$ ranges over $\mathfrak{C}_{n}$ is equal to

$$
\prod_{1 \leq i \leq n}\left(1-t^{1 / 2} x_{i}\right)\left(1+t^{1 / 2} x_{i}\right) \prod_{1 \leq i<j \leq n}\left(1-x_{i} x_{j}\right)\left(x_{i}-x_{j}\right),
$$

which is equal to the product side of (CD). The proofs of $(3.1 \mathrm{bd})$ and $(3.1 \mathrm{bc})$ are similar.

\section{The Sum Sides}

To describe the subsets of $\mathfrak{B}_{n}$ and $\mathfrak{C}_{n}$ that correspond to the sum sides of (BD), (BC), and (CD), we need to study these sets of digraphs in more detail. The positive subtournaments of digraphs in $\mathfrak{B}_{n} \cup \mathfrak{C}_{n}$ will be important in this study, and we shall also use partially ordered sets.

\section{Transitive Tournaments and Permutations}

Suppose $\mathfrak{g}$ is a digraph on $V$ such that no two vertices of $V$ have the same outdegree in $\mathfrak{g}$. We claim that $\mathfrak{g}$ is complete and transitive, or in other words, that it is a transitive tournament. This is obvious (and vacuous) if $|V|=1$. Otherwise, observe that the possible out-degrees of any vertex in any digraph on $V$ are 0 , $1, \ldots,|V|-1$; therefore $\mathfrak{g}$ must have exactly one vertex of each possible outdegree. If $i$ is the vertex whose out-degree is $|V|-1$, then $i$ is not part of any cycle and there is an arc between $i$ and any other vertex in $\mathfrak{g}$. The restriction of $\mathfrak{g}$ to $V \backslash\{i\}$ has no two vertices with the same out-degree, and if it is complete and transitive, then so is $\mathfrak{g}$. Conversely, suppose $\mathfrak{g}$ is complete and transitive. If $(i, j) \in \mathfrak{g}$, then we have $(i, k) \in \mathfrak{g}$ whenever $(j, k) \in \mathfrak{g}$. Since $(j, j)$ is not in $\mathfrak{g}$, we see that $\{k:(j, k) \in \mathfrak{g}\}$ is a proper subset of $\{k:(i, k) \in \mathfrak{g}\}$. We conclude that 
$o(i, \mathfrak{g})>o(j, \mathfrak{g})$. This means that no two vertices have the same out-degree in $\mathfrak{g}$. We have shown that a digraph $\mathfrak{g}$ on (a finite set) $V$ is complete and transitive if and only if $\{o(i, \mathfrak{g}): i \in V\}=\{0,1, \ldots,|V|-1\}$.

The above result allows us to identify transitive tournaments $\mathfrak{g}$ on $[n]$ with permutations $\sigma \in S_{n}$. Given such an $\mathfrak{g}$, define $\sigma(i)$ to be the unique $j$ such that $o(j, \mathfrak{g})=n-i$. Then $\mathfrak{g}=\{(\sigma(i), \sigma(j)): 1 \leq i<j \leq n\}$. This correspondence between permutations and digraphs is of great importance here and in $[\mathrm{G}]$ and $[\mathrm{B}]$.

\section{Alternative Description of $\mathfrak{g}$ When $\mathfrak{g}^{+}$is Transitive}

Let $\mathfrak{g}$ be a digraph in $\mathfrak{B}_{n} \cup \mathfrak{C}_{n}$ whose positive subtournament $\mathfrak{g}^{+}$is transitive. There is a unique permutation $\sigma=\sigma(\mathfrak{g}) \in S_{n}$ such that $o\left(\sigma(i), \mathfrak{g}^{+}\right)=n-i$ for each $i \in[n]$. Observe that $\sigma$ completely determines all arcs in $\mathfrak{g}$ between two positive vertices or between two negative vertices. We now introduce some sets that, together with $\sigma$, will determine all arcs in $\mathfrak{g}$ not between two positive or two negative vertices.

First, we introduce $\left(\begin{array}{c}{[n]} \\ 2\end{array}\right)$ (" $[n]$ choose 2 ") and $\left\langle\begin{array}{c}n] \\ 2\end{array}\right\rangle$ ("[n] repeat-choose 2" or "[n] choose 2 with repetition"): $\left(\begin{array}{c}{[n]} \\ 2\end{array}\right)=\{(i, j): 1 \leq i<j \leq n\}$ and $\left\langle\begin{array}{c}{[n]} \\ 2\end{array}\right\rangle=\{(i, j): 1 \leq$ $i \leq j \leq n\}$. Then we define

$$
\begin{aligned}
\tau=\tau(\mathfrak{g}) & =\left\{(i, j) \in\left(\begin{array}{c}
{[n]} \\
2
\end{array}\right):(\sigma(i),-\sigma(j)) \in \mathfrak{g}\right\}, \\
\bar{\tau}=\bar{\tau}(\mathfrak{g}) & =\tau(\mathfrak{g}) \cup\{(i, i):(\sigma(i), 0) \in \mathfrak{g})\}, \\
\omega_{0}=\omega_{0}(\mathfrak{g}) & =\{i:(\sigma(i), 0) \in \mathfrak{g}\},
\end{aligned}
$$

and for $\mathfrak{g} \in \mathfrak{C}_{n}$

$$
\omega_{ \pm}=\omega_{ \pm}(\mathfrak{g})=\{i:(\sigma(i),-\sigma(i)) \in \mathfrak{g}\} .
$$

We shall use these sets throughout Sections 6 and 7 . For instance, if $\mathfrak{g}$ is the digraph in Figure 3.2, then $\mathfrak{g}^{+}$is transitive with $\sigma(\mathfrak{g})=(23), \bar{\tau}(\mathfrak{g})=\{(1,2),(1,3),(2,2)\}$, and $\omega_{0}(\mathfrak{g})=\{2\}$.

We are going to define a partial order on $\left(\begin{array}{c}{[n]} \\ 2\end{array}\right)$ and $\left\langle\begin{array}{c}n \\ 2\end{array}\right)$. Recall ([St], Chapter 3 ) that a partial order is a binary relation $\preceq$ that is reflexive ( $x \preceq x$ for any $x$ ), antisymmetric (if $x \preceq y$ and $y \preceq x$, then $x=y$ ), and transitive (if $x \preceq y$ and $y \preceq z$, then $x \preceq z$ ). One writes $x \prec y$ if $x \preceq y$ and $x \neq y$; also, if $x \preceq y$ or $x \prec y$, one may write $y \succeq x$ or $y \succ x$. A set on which a partial order is defined is called a partially ordered set, or poset for short.

Let $P$ be a poset with partial order $\preceq$. A subset $Q$ of $P$ is an order ideal with respect to $\preceq$ if we have $x \in Q$ whenever $x \preceq y$ and $y \in Q$. A dual order ideal is a subset $Q$ of $P$ such that $y \in Q$ whenever $x \preceq y$ and $x \in Q$. Evidently $Q$ is a dual order ideal of $P$ if and only if $P \backslash Q$ is an order ideal.

Given ordered pairs of integers $(i, j)$ and $(k, l)$, we say that $(i, j) \preceq(k, l)$ if and only if $i \leq k$ and $j \leq l$. It is easy to see that $\preceq$ is a partial order on $\left(\begin{array}{c}{[n]} \\ 2\end{array}\right)$ and $\left\langle\begin{array}{c}{[n]} \\ 2\end{array}\right\rangle$. We shall visualize $\left(\begin{array}{c}{[n]} \\ 2\end{array}\right)$ and $\left\langle\begin{array}{c}{[n]} \\ 2\end{array}\right\rangle$ as in Figure 4.1 , which shows $T_{1}=\{(1,2)$, $(2,3)\} \subseteq\left(\begin{array}{c}{[3]} \\ 2\end{array}\right)$ and $T_{2}=\{(1,1),(1,2),(2,2)\} \subseteq\left\langle\begin{array}{c}{[3]} \\ 2\end{array}\right\rangle$. Observe that $T_{2}$ is an order ideal and $T_{1}$ is not. 

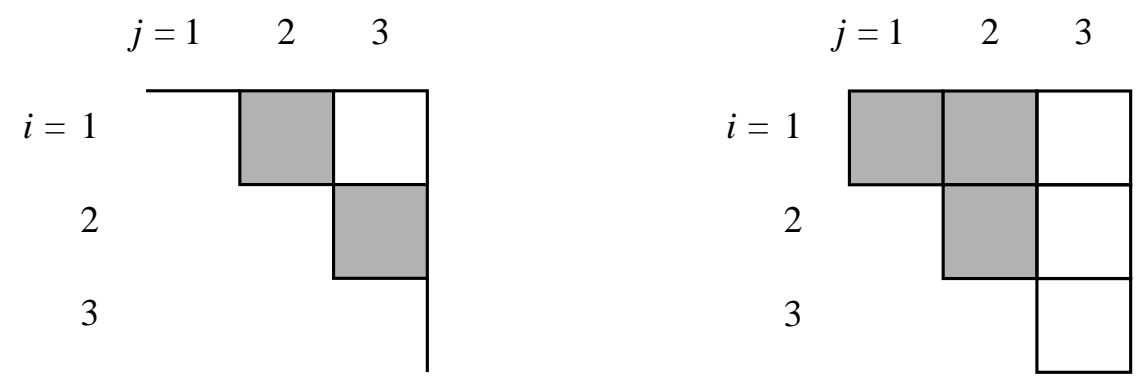

Figure 4.1

The map $\mathfrak{b} \mapsto(\sigma(\mathfrak{b}), \bar{\tau}(\mathfrak{b}))$ is a bijection of $\left\{\mathfrak{b} \in \mathfrak{B}_{n}: \mathfrak{b}^{+}\right.$transitive $\}$onto $S_{n} \times$ Pow $\left\langle\begin{array}{c}{[n]} \\ 2\end{array}\right\rangle$, where Pow $X$ denotes the set of all subsets of $X$. We also have bijections $\mathfrak{c} \mapsto\left(\sigma(\mathfrak{c}), \bar{\tau}(\mathfrak{c}), \omega_{ \pm}(\mathfrak{c})\right)$ and $\mathfrak{c} \mapsto\left(\sigma(\mathfrak{c}), \tau(\mathfrak{c}), \omega_{0}(\mathfrak{c}), \omega_{ \pm}(\mathfrak{c})\right)$ of $\left\{\mathfrak{c} \in \mathfrak{C}_{n}: \mathfrak{c}^{+}\right.$transitive $\}$ onto $S_{n} \times$ Pow $\left\langle\begin{array}{c}{[n]} \\ 2\end{array}\right) \times$ Pow $[n]$ and $S_{n} \times$ Pow $\left(\begin{array}{c}{[n]} \\ 2\end{array}\right) \times$ Pow $[n] \times$ Pow $[n]$ respectively. In Chapter 2 of $[S]$, it is shown that $\mathfrak{b} \in \mathfrak{B}_{n}$ is transitive if and only if $\mathfrak{b}^{+}$is transitive and $\bar{\tau}(\mathfrak{b})$ is an order ideal of $\left\langle\begin{array}{c}{[n]} \\ 2\end{array}\right\rangle$. Similarly, $\mathfrak{c} \in \mathfrak{C}_{n}$ is transitive if and only if $\mathfrak{c}^{+}$is transitive, $\bar{\tau}(\mathfrak{c})$ is an order ideal of $\left\langle\begin{array}{c}{[n]} \\ 2\end{array}\right\rangle$, and $\omega_{0}(\mathfrak{c})=\omega_{ \pm}(\mathfrak{c})$.

The last concept we need in this section is that of interchangeability. Let $T$ be a subset of $\left(\begin{array}{c}{[n]} \\ 2\end{array}\right)$ or of $\left\langle\begin{array}{c}{[n]} \\ 2\end{array}\right\rangle$ and let $i$ and $j$ be distinct integers in $[n]$. Suppose without loss of generality that $i<j$. We say $i$ and $j$ are interchangeable in $T$ if the following conditions hold:

For $1 \leq k<i,(k, i) \in T$ if and only if $(k, j) \in T$.

For $i<k<j,(i, k) \in T$ if and only if $(k, j) \in T$.

For $j<k \leq n,(i, k) \in T$ if and only if $(j, k) \in T$.

$(i, i) \in T$ if and only if $(j, j) \in T$ (this is vacuous if $\left.T \subseteq\left(\begin{array}{c}{[n]} \\ 2\end{array}\right)\right)$.

We are ready to define the sets of digraphs that will index the sum sides of our three generalizations of Weyl's formula. These sets are $\mathfrak{B}_{n}^{*} \subset \mathfrak{B}_{n}, \mathfrak{C}_{n}^{*} \subset \mathfrak{C}_{n}$, and $\mathfrak{C}_{n}^{* *} \subset \mathfrak{C}_{n}$, defined as follows:

$\mathfrak{b} \in \mathfrak{B}_{n}^{*}$ if $\mathfrak{b}^{+}$is transitive, $\tau(\mathfrak{b})$ is an order ideal of $\left(\begin{array}{c}{[n]} \\ 2\end{array}\right)$, and

$$
o(\sigma(1), \mathfrak{b})>o(\sigma(2), \mathfrak{b})>\cdots>o(\sigma(n), \mathfrak{b})
$$

$\mathfrak{c} \in \mathfrak{C}_{n}^{*}$ if $\mathfrak{c}^{+}$is transitive, $\bar{\tau}(\mathfrak{c})$ is an order ideal of $\left\langle\begin{array}{c}{[n]} \\ 2\end{array}\right\rangle$, and

$$
o(\sigma(1), \mathfrak{c})>o(\sigma(2), \mathfrak{c})>\cdots>o(\sigma(n), \mathfrak{c})
$$

$\mathfrak{c} \in \mathfrak{C}_{n}^{* *}$ if $\mathfrak{c}^{+}$is transitive, $\tau(\mathfrak{c})$ is an order ideal of $\left(\begin{array}{c}{[n]} \\ 2\end{array}\right)$,

$$
o(\sigma(1), \mathfrak{c})>o(\sigma(2), \mathfrak{c})>\cdots>o(\sigma(n), \mathfrak{c})
$$

and $\omega_{0}(\mathfrak{c}) \subseteq \omega_{ \pm}(\mathfrak{c})$, with $\omega_{ \pm}(\mathfrak{c}) \backslash \omega_{0}(\mathfrak{c})$ being a disjoint union of pairs $\{j, j+1\}$ such that the elements of each such pair are interchangeable in $\tau(\mathfrak{c})$.

Section 6 will be devoted to the proof of 
Lemma 4.1. ([S], Lemma 3.3.1) We have

$$
\sum_{\mathfrak{b} \in \mathfrak{B}_{n} \backslash \mathfrak{B}_{n}^{*}} \hat{w}(\mathfrak{b})=\sum_{\mathfrak{c} \in \mathfrak{C}_{n} \backslash \mathfrak{C}_{n}^{*}} \tilde{w}(\mathfrak{c})=\sum_{\mathfrak{c} \in \mathfrak{C}_{n} \backslash \mathfrak{C}_{n}^{* *}} \check{w}(\mathfrak{c})=0
$$

for all $n \geq 1$.

In Section 7, we shall prove

Lemma 4.2. ([S], Lemma 3.4.1) We have

$$
\begin{aligned}
& \sum_{\mathfrak{b} \in \mathfrak{B}_{n}^{*}} \hat{w}(\mathfrak{b})=\sum_{\substack{\lambda \in P_{-1,0}(n) \\
\sigma \in S_{n}}}(-1)^{|\lambda|-|\mu| / 2} t^{|\lambda|-|\mu|}\left(1-t^{2}\right)^{y(\lambda)}(-1)^{\sigma} x^{\sigma\left(\lambda+\delta_{n}\right)} ; \\
& \sum_{\mathfrak{c} \in \mathfrak{C}_{n}^{*}} \tilde{w}(\mathfrak{c}) \\
& \quad=\sum_{\substack{\lambda \in P_{0,1}(n) \\
\sigma \in S_{n}}}(-1)^{(|\nu|+p(\nu)) / 2} t^{|\lambda|-|\nu|}(1-t)^{\chi\left(\exists i, \lambda_{i}=i\right)}\left(1-t^{2}\right)^{z(\lambda)}(-1)^{\sigma} x^{\sigma\left(\lambda+\delta_{n}\right)} ; \\
& \sum_{\mathfrak{c} \in \mathfrak{C}_{n}^{* *}} \check{w}(\mathfrak{c}) \sum_{\substack{\lambda \in P_{-1,1}(n) \\
\sigma \in S_{n}}}(-1)^{|\kappa| / 2+q(\lambda)} t^{(|\lambda|-|\kappa|) / 2}(1-t)^{\chi\left(\exists i, \lambda_{i}=i\right)}\left(1-t^{2}\right)^{z(\lambda)}(-1)^{\sigma} x^{\sigma\left(\lambda+\delta_{n}\right)},
\end{aligned}
$$

for all $n \geq 1$, where $\mu, \nu, \kappa, y, z$, and $q$ are as defined in Section 2.

Theorems 2.2 and 2.4 follow from these lemmas and from (3.1bd), (3.1bc), and (3.1cd).

\section{Lemmas on Order Ideals and Partitions}

Before we begin to prove Lemmas 4.1 and 4.2, we shall need some more results concerning order ideals, partitions, and interchangeability.

Let $T$ be a subset of $\left\langle\begin{array}{c}{[n]} \\ 2\end{array}\right\rangle$. For any $i \in[n]$ and any $t \geq-1$, we define

$$
\#_{t}(i, T)=|\{j: j<i,(j, i) \in T\}|+|\{j: j>i,(i, j) \in T\}|+(t+1) \chi((i, i) \in T) .
$$

In other words, $\#_{t}(i, T)$ is the number of ordered pairs in $T$ with at least one component equal to $i$, plus $t$ times the number of ordered pairs in $T$ with both components equal to $i$. Observe that $\#_{t}$ is independent of $t$ whenever $T \subseteq\left(\begin{array}{c}{[n]} \\ 2\end{array}\right)$. We shall write \# instead of $\#_{t}$ in case $T$ is known to be a subset of $\left(\begin{array}{c}{[n]} \\ 2\end{array}\right)$. We remark that $\#\left(i, T \cap\left(\begin{array}{c}{[n]} \\ 2\end{array}\right)\right)=\#_{-1}(i, T)$ for any $T \subseteq\left\langle\begin{array}{c}{[n]} \\ 2\end{array}\right\rangle$.

The following lemma establishes an important correspondence between partitions and order ideals of $\left(\begin{array}{c}{[n]} \\ 2\end{array}\right)$ and $\left\langle\begin{array}{c}{[n]} \\ 2\end{array}\right\rangle$. 
Lemma 5.1. ([S], Lemma 2.3.3) (a) If $T$ is an order ideal of $\left(\begin{array}{c}{[n]} \\ 2\end{array}\right)$ and $\lambda=$ $(\#(1, T), \ldots, \#(n, T))$, then $\lambda \in P_{-1}(n)$ and $|T|=|\lambda| / 2$.

(b) If $T$ is an order ideal of $\left\langle\begin{array}{c}{[n]} \\ 2\end{array}\right\rangle$ and $\lambda=\left(\#_{t}(1, T), \ldots, \#_{t}(n, T)\right)$, then $\lambda \in P_{t}(n)$ and, if $t \geq 0$, then $|T|=(|\lambda|+(1-t) p(\lambda)) / 2$.

Proof. (a) Suppose $T$ is an order ideal of $\left(\begin{array}{c}{[n]} \\ 2\end{array}\right)$. Consider the set

$$
D=\{(i, j-1),(j, i):(i, j) \in T\} .
$$

We visualize $D$ as being made of two copies of $T$, one copy reflected about the diagonal of $\mathbf{N}^{2}$ and glued to the other as shown in Figure 5.1.

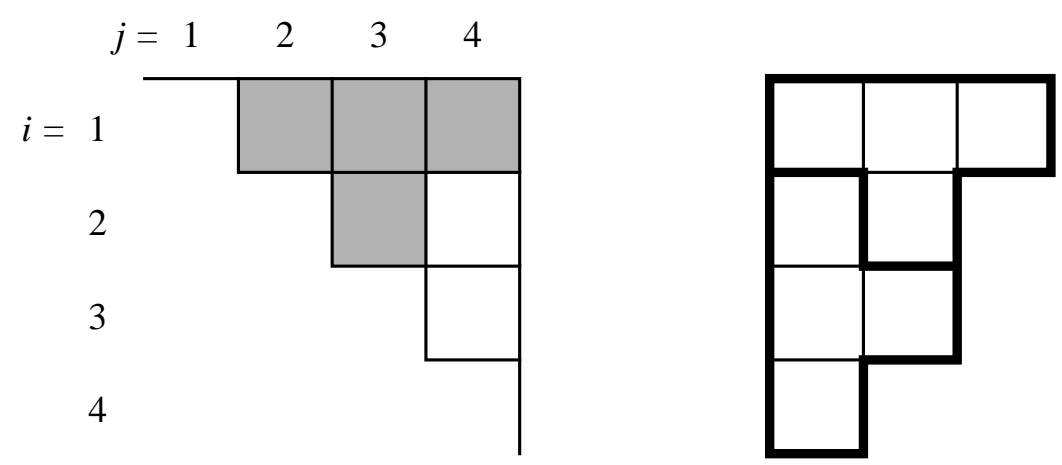

FiguRE 5.1

We claim that $D$ is the Ferrers diagram of a partition $\lambda \in P_{-1}(n)$. Indeed, if $T$ is an order ideal of $\left(\begin{array}{c}{\left[\begin{array}{c}n \\ 2\end{array}\right)} \\ )\end{array}\right)$, then $T$ is of the form

$$
\left\{(1,2),(1,3), \ldots,\left(1,1+\alpha_{1}\right),(2,3), \ldots,\left(2,2+\alpha_{2}\right), \ldots,(p, p+1), \ldots,\left(p, p+\alpha_{p}\right)\right\}
$$

for some $p \leq n-1$ and $\alpha_{1}>\alpha_{2}>\cdots>\alpha_{p} \geq 1$, and $D$ is the Ferrers diagram of $\lambda=\left(\alpha_{1}-1, \ldots, \alpha_{p}-1 \mid \alpha_{1}, \ldots, \alpha_{p}\right)$. It is clear that $|T|=|\lambda| / 2$. So we can complete the proof of (a) by showing that $\lambda_{i}=\#(i, T)$ for each $i \in[n]$. This is not hard to see. Given $(i, j) \in D$, either $j<i$ and $(i, j)$ corresponds to $(j, i) \in T$, or $j \geq i$ and $(i, j)$ corresponds to $(i, j+1) \in T$; so each of the $\lambda_{i}$ squares in the $i$ th row of $D$ corresponds to a distinct ordered pair in $T$ with one component equal to $i$, and this is clearly a one-to-one correspondence.

(b) Suppose $T$ is an order ideal of $\left\langle\begin{array}{c}{[n]} \\ 2\end{array}\right\rangle$. Then $T \cap\left(\begin{array}{c}{[n]} \\ 2\end{array}\right)$ is an order ideal of $\left(\begin{array}{c}{[n]} \\ 2\end{array}\right)$, and since $\#_{-1}(i, T)=\#\left(i, T \cap\left(\begin{array}{c}{[n]} \\ 2\end{array}\right)\right)$ for all $i \in[n]$, the case $t=-1$ reduces to the case considered in (a).

Now suppose $t=0$. Consider the set

$$
D=\{(i, j),(j, i):(i, j) \in T\}
$$

we think of $D$ as being made of two copies of $T$, one copy being reflected about the diagonal and glued to the other with overlap along the diagonal. Observe that $D$ is the Ferrers diagram of a partition $\lambda \in P_{0}(n)$. We see that $p(\lambda)$ is the largest 
$i$ such that $(i, i) \in T$, and that $|T|=(\lambda+p(\lambda)) / 2$. To see that $\lambda_{i}=\#_{0}(i, T)$ for each $i$, observe that for any given $(i, j) \in D$, either $j<i$ and $(j, i) \in T$, or $j \geq i$ and $(i, j) \in T$. Each of the $\lambda_{i}$ squares in the $i$ th row of $D$ corresponds to a distinct ordered pair in $T$ with at least one component equal to $i$. This takes care of the case $t=0$.

If $t \geq 1$, then let

$$
D=\{(i, j+t),(j, i):(i, j) \in T\} \cup\{(i, i+1), \ldots,(i, i+t-1): 1 \leq i \leq p\},
$$

where $p$ is the largest $i$ such that $(i, i) \in T$. In this case, $D$ is made of two copies of $T$, with one copy reflected about the diagonal as before, but the copies are separated by a diagonal strip of width $t-1$ and length $p$. Figure 5.2 shows an example in the case $t=2$.
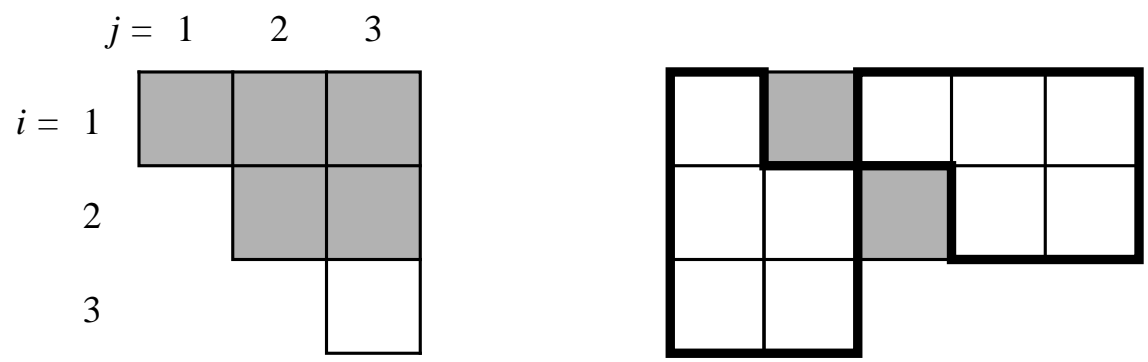

FiguRE 5.2

We find that $D$ is the Ferrers diagram of a partition $\lambda \in P_{t}(n)$, that $p(\lambda)=p$, and that $|T|=(|\lambda|+(1-t) p(\lambda)) / 2$. To see that $\lambda_{i}=\#_{t}(i, T)$ for each $i$, observe that if $(i, j) \in D$, then either: $j<i$ and $(j, i) \in T$, or $i \leq j \leq i+t$ and $(i, i) \in T$, or $j>i+t$ and $(i, j-t) \in T$. So the $i$ th row of $D$ contains one square for each ordered pair in $T$ having just one component equal to $i$, and $t+1$ squares for the ordered pair $(i, i)$ if it is in $T$.

The correspondence described by Lemma 5.1(a) is one-to-one, as is that described by Lemma 5.1(b) for $t \geq 0$. Given $\lambda \in P_{-1}(n)$, then $T=\left(\begin{array}{c}{[n]} \\ 2\end{array}\right) \cap\{(i, j+1):(i, j) \in$ $D(\lambda)\}$ is the unique order ideal of $\left(\begin{array}{c}{[n]} \\ 2\end{array}\right)$ for which $\#(i, T)=\lambda_{i}$ for all $i$; if $\lambda \in P_{t}(n)$ and $t \geq 0$, then $T=\left\langle\begin{array}{c}{[n]} \\ 2\end{array}\right\rangle \cap\{(i, j-t):(i, j) \in D(\lambda)\}$ is the unique order ideal of $\left\langle\begin{array}{c}{[n]} \\ 2\end{array}\right\rangle$ such that $\#_{t}(i, T)=\lambda_{i}$ for all $i$.

The next lemma we shall prove concerns interchangeability. Consider the subset $\tau$ of $\left(\begin{array}{c}{[6]} \\ 2\end{array}\right)$ shown in Figure 5.3. The arrows indicate which pairs of elements of $\left(\begin{array}{c}66 \\ 2\end{array}\right)$ must be examined for membership in $\tau$ to decide whether 2 and 4 are interchangeable in $\tau$. For each arrow, the element of $\left(\begin{array}{c}6] \\ 2\end{array}\right)$ at one end belongs to $\tau$ if and only if the element at the other end belongs to $\tau$; this shows that 2 and 4 are interchangeable. 


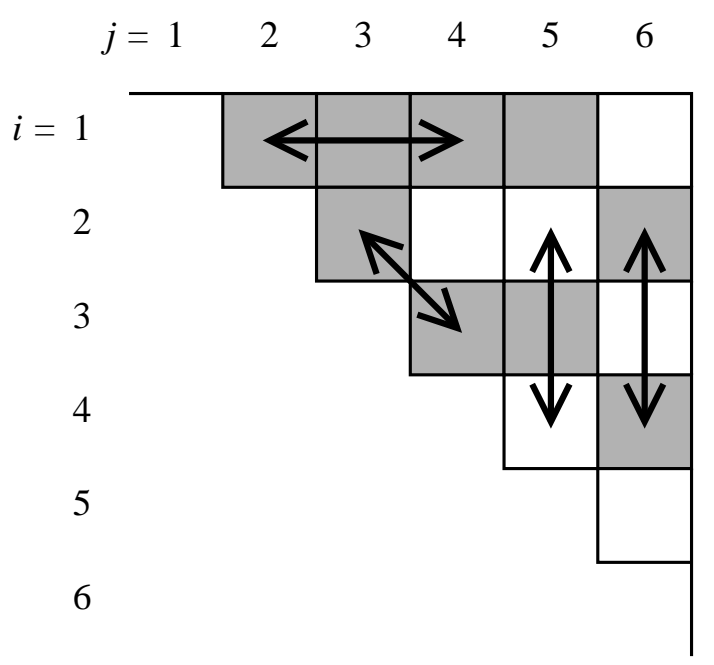

Figure 5.3

It is easy to see that if $i$ and $j$ are interchangeable in $T \subseteq\left\langle\begin{array}{c}{[n]} \\ 2\end{array}\right\rangle$, then \# $\#_{t}(i, T)=$ $\#_{t}(j, T)$ for any $t \geq-1$, and if $i$ and $j$ are interchangeable in $T \subseteq\left(\begin{array}{c}{[n]} \\ 2\end{array}\right)$, then $\#(i, T)=\#(j, T)$. For instance, we have $\#(2, \tau)=\#(4, \tau)$ when $\tau$ is as in Figure 5.3. We also have $\#(5, \tau)=\#(6, \tau)$ in this case, although 5 and 6 are not interchangeable in $\tau$. The following lemma tells us that this cannot happen when $T$ is an order ideal:

Lemma 5.2. ([S], Lemma 3.2.1) (a) If $T$ is an order ideal of $\left(\begin{array}{c}{[n]} \\ 2\end{array}\right)$ and \# $(i, T)=$ $\#(j, T)$, then $i$ and $j$ are interchangeable in $T$.

(b) If $T$ is an order ideal of $\left\langle\begin{array}{c}{[n]} \\ 2\end{array}\right\rangle$ and $\#_{t}(i, T)=\#_{t}(j, T)$ for some $t \geq 0$, then $i$ and $j$ are interchangeable in $T$.

Proof. Assuming the hypothesis of (a), with $i<j$, let $r$ be the common value of $\#(i, T)$ and $\#(j, T)$. It is easy to see that $r \leq i-1$ if $(i, j) \notin T$ and $r \geq j-1$ if $(i, j) \in T$. In the former case, we find that $(k, i) \in T$ if and only if $(k, j) \in T$ if and only if $1 \leq k \leq r$, while any other $(k, l)$ with $k$ or $l$ equal to $i$ or $j$ is not in $T$. The latter case is similar, except that we have: $(k, i),(k, j) \in T$ whenever $1 \leq k<i$; $(i, k),(k, j) \in T$ whenever $i<k<j$; for $k>j,(i, k) \in T$ if and only if $(j, k) \in T$ if and only if $j+1 \leq k \leq r+1$. This proves (a); the proof of (b) is almost identical. We mention that the condition $t \geq 0$ in (b) is necessary to ensure that $(i, i)$ and $(j, j)$ are counted by $\#_{t}(i, T)$ and $\#_{t}(j, T)$ whenever they belong to $T$.

Suppose $\bar{T}$ is a subset of $\left\langle\begin{array}{c}{[n]} \\ 2\end{array}\right\rangle$ and $T=\bar{T} \cap\left(\begin{array}{c}{[n]} \\ 2\end{array}\right)$. Clearly, $T$ is an order ideal of $\left(\begin{array}{c}{[n]} \\ 2\end{array}\right)$ if $\bar{T}$ is an order ideal of $\left\langle\begin{array}{c}{[n]} \\ 2\end{array}\right\rangle$, but the converse does not hold. For $\bar{T}$ to be an order ideal of $\left\langle\begin{array}{c}{[n]} \\ 2\end{array}\right\rangle$, it is necessary that $T$ be an order ideal of $\left(\begin{array}{c}{[n]} \\ 2\end{array}\right)$, but not sufficient. The last lemma of this section gives the additional conditions we need in order for $\bar{T}$ to be an order ideal. To state it, we need to introduce what we shall call extreme points of an order ideal of a poset. 
Let $P$ be a poset and $T$ an order ideal of $P$. We say that $p \in T$ is an inner extreme point of $T$ if $T \backslash\{p\}$ is an order ideal of $P$. Meanwhile, $p$ is an outer extreme point of $T$ if $p \notin T$ and $T \cup\{p\}$ is an order ideal of $P$. We denote by $I(T)$ and $O(T)$ the sets of all inner and outer extreme points of $T$. Evidently $I(T)$ is the smallest set $S$ such that $T=\{p: p \preceq s$ for some $s \in S\}$, while $O(T)$ is the smallest $S$ for which $P \backslash T=\{q: q \succeq s$ for some $s \in S\}$. Figure 5.4 portrays order

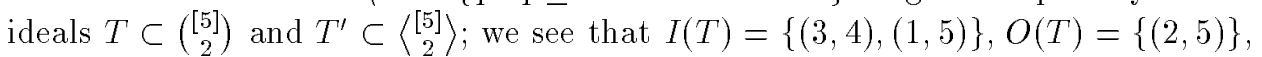
$I\left(T^{\prime}\right)=\{(2,3),(1,5)\}$, and $O\left(T^{\prime}\right)=\{(3,3),(2,4)\}$.
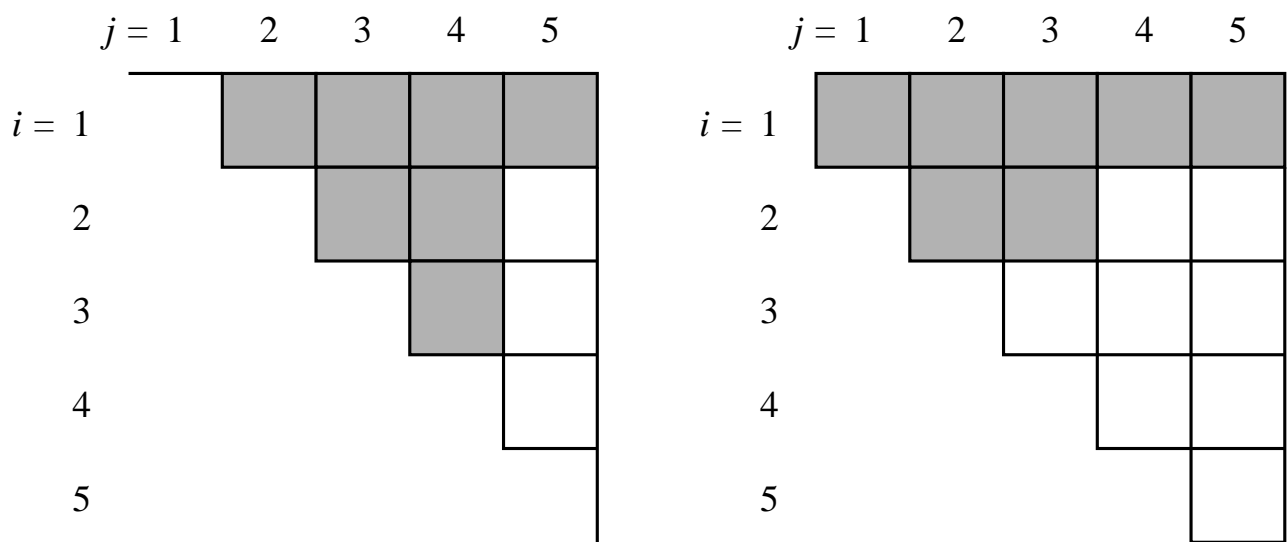

Figure 5.4

We are ready to state and prove:

Lemma 5.3. ([S], Lemma 3.2.2) Let $\bar{\tau} \subseteq\left\langle\begin{array}{c}{[n]} \\ 2\end{array}\right\rangle$ and $\tau=\bar{\tau} \cap\left(\begin{array}{c}{[n]} \\ 2\end{array}\right)$. Then $\bar{\tau}$ is an order ideal of $\left\langle\begin{array}{c}{[n]} \\ 2\end{array}\right\rangle$ if and only if all the following conditions hold:

(i) $\tau$ is an order ideal of $\left(\begin{array}{c}{[n]} \\ 2\end{array}\right)$.

(ii) For all $(i, j) \in I(\tau),(i, i) \in \bar{\tau}$ or $(j, j) \in \bar{\tau}$.

(iii) For all $(i, j) \in O(\tau),(i, i) \notin \bar{\tau}$ or $(j, j) \notin \bar{\tau}$.

(iv) $\{i:(i, i) \in \bar{\tau}\}$ is of the form $\{1,2, \ldots, r\}$ for some $r, 0 \leq r \leq n$.

Furthermore, if (i)-(iii) hold and (iv) does not, there exists $i \in[n]$ such that $(i, i) \notin \bar{\tau},(i+1, i+1) \in \bar{\tau}$, and $i$ and $i+1$ are interchangeable in $\tau$.

Proof. "Only if": Suppose $\bar{\tau}$ is an order ideal of $\left\langle\begin{array}{c}{[n]} \\ 2\end{array}\right)$. If $(i, j) \in \tau$ and $(k, l) \prec(i, j)$, then $(k, l) \in \bar{\tau}$; if $(k, l) \in\left(\begin{array}{c}{[n]} \\ 2\end{array}\right)$, then $(k, l) \in \tau$. Therefore (i) holds. If $(i, j) \in I(\tau)$, then $(i, j) \in \tau$, which implies $(i, i) \in \bar{\tau}$, so (ii) holds. Similarly, for $(i, j) \in O(\tau)$ we have $(i, j) \notin \bar{\tau}$, which means $(j, j) \notin \bar{\tau}$, and (iii) holds. If $(i, i) \in \bar{\tau}$, then $(j, j) \in \bar{\tau}$ for any $j \leq i$, so (iv) holds.

"If": Suppose $\bar{\tau}$ is not an order ideal of $\left\langle\begin{array}{c}{[n]} \\ 2\end{array}\right)$. Then there exist $i, j \in[n]$ with $i \leq j$ and either $(i, j) \notin \bar{\tau},(i, j+1) \in \bar{\tau}$ or $(i-1, j) \notin \bar{\tau},(i, j) \in \bar{\tau}$. If $i<j$, then $\tau$ is not an order ideal of $\left(\begin{array}{c}{[n]} \\ 2\end{array}\right)$. If $\tau$ is an order ideal of $\left(\begin{array}{c}{[n]} \\ 2\end{array}\right)$ but $(i, i) \notin \bar{\tau},(i, i+1) \in \bar{\tau}$ for some $i$, then there exist $j, k$ with $k>j \geq i$ and $(j, k) \in I(\tau)$. If either $(j, j)$ or $(k, k)$ is in $\bar{\tau}$, then (iv) is violated; otherwise (ii) does not hold. If $\tau$ is an order 
ideal of $\left(\begin{array}{c}{[n]} \\ 2\end{array}\right)$ and $(i-1, i) \notin \bar{\tau},(i, i) \in \bar{\tau}$, then we have $(j, k) \in O(\tau)$ for some $j, k$ with $i \geq k>j$. If $(j, j) \notin \bar{\tau}$ or $(k, k) \notin \bar{\tau}$, then (iv) does not hold; otherwise (iii) fails.

"Furthermore": Suppose $\bar{\tau} \subseteq\left\langle\begin{array}{c}{[n]} \\ 2\end{array}\right\rangle$ is such that (i)-(iii) hold and (iv) does not. Choose $i$ such that $(i, i) \notin \bar{\tau},(\bar{i}+1, i+1) \in \bar{\tau}$. If $i$ and $i+1$ are interchangeable in $\tau$, we are done. If not, then either $(i, j) \in \tau,(i+1, j) \notin \tau$ for some $j \geq i+2$ or $(k, i) \in \tau,(k, i+1) \notin \tau$ for some $k \leq i-1$. In the former case, choose the smallest $j$ with this property. Then $(i+1, j) \in O(\tau)$; since (iii) holds and $(i+1, i+1) \in \bar{\tau}$, we must have $(j, j) \notin \bar{\tau}$. There is some $l \geq j$ such that $(i, l) \in I(\tau)$, and since (ii) holds and $(i, i) \notin \bar{\tau}$, we must have $(l, l) \in \bar{\tau}$, which tells us $l>j$. We find that $\#(q, \tau)=i$ for each $q$ with $j \leq q \leq l$; Lemma 5.2(a) tells us that $j, j+1, \ldots, l$ are pairwise interchangeable in $\tau$, and for some $q$ with $j \leq q<l$, we must have $(q, q) \notin \bar{\tau},(q+1, q+1) \in \bar{\tau}$. A similar argument applies in the latter case.

Figure 5.5 shows a subset $\bar{\tau}$ of $\left\langle\begin{array}{c}{[7]} \\ 2\end{array}\right\rangle$ for which only condition (iv) fails. The bold line separates $\left(\begin{array}{c}{[7]} \\ 2\end{array}\right)$ from pairs of the form $(i, i)$. We have $(6,6) \notin \bar{\tau},(7,7) \in \bar{\tau}$, but 6 and 7 are not interchangeable in $\tau$. We see that $(2,7) \in O(\tau)$ and $(4,6) \in I(\tau)$; 3 and 4 are interchangeable in $\tau$ and $(3,3) \notin \bar{\tau},(4,4) \in \bar{\tau}$.

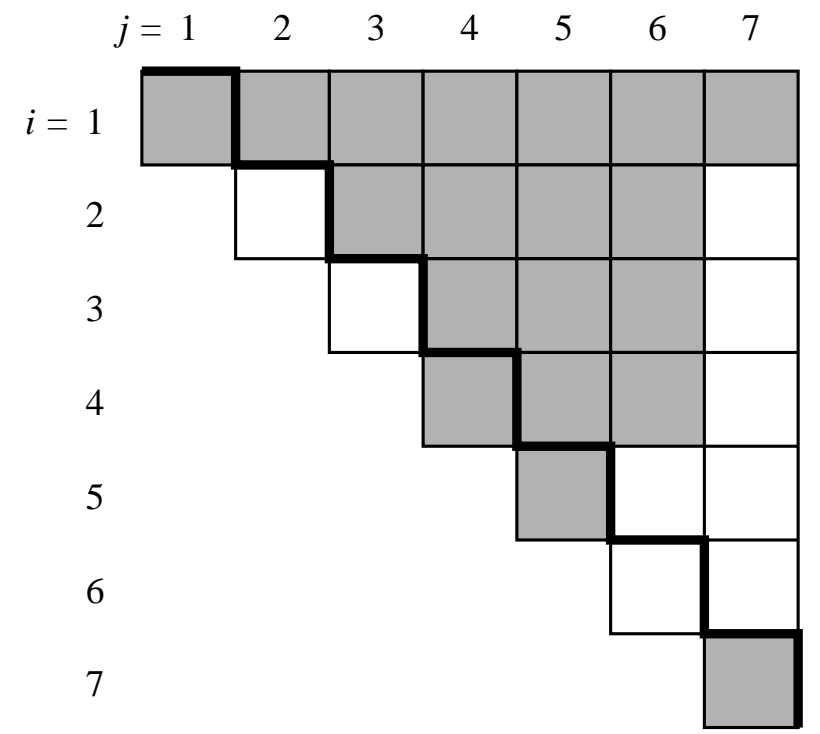

Figure 5.5

\section{Cancellation}

Before beginning the proof of Lemma 4.1, we need to introduce some operations on digraphs. We shall use these operations to "pair off" the digraphs in $\mathfrak{B}_{n} \backslash \mathfrak{B}_{n}^{*}$, $\mathfrak{C}_{n} \backslash \mathfrak{C}_{n}^{*}$, and $\mathfrak{C}_{n} \backslash \mathfrak{C}_{n}^{* *}$. Each such digraph will be paired with another, whose weight is -1 times that of the first. 


\section{Arc Reversal and Vertex Interchange}

Given a digraph $\mathfrak{g}$ on $V$, we can obtain other digraphs on $V$ by reversing some of the arcs in $\mathfrak{g}$. Specifically, we reverse the arcs in a subset $R$ of $\mathfrak{g}$ by replacing $R$ with $R^{\prime}=\{(j, i):(i, j) \in R\}$. This gives us the digraph $\mathfrak{g}^{\prime}=R^{\prime} \cup \mathfrak{g} \backslash R$. If $R$ is a disjoint union of cycles, then we have $o\left(i, \mathfrak{g}^{\prime}\right)=o(i, \mathfrak{g})$ for every $i \in V$. In this case, $R^{\prime}$ is also a disjoint union of cycles. Whatever $R$ may be, we recover $\mathfrak{g}$ from $\mathfrak{g}^{\prime}$ by reversing the arcs in $R^{\prime}$.

Another way of obtaining a new digraph on $V$ from an existing one is by interchanging vertices. Let $\mathfrak{g}$ be a digraph on $V$ and let $p, q \in V$. Let $\phi_{p q}$ be the bijection on $V$ that interchanges $p$ and $q$ while fixing everything else in $V$. Then $\mathfrak{g}_{p q}=\left\{\left(\phi_{p q}(i), \phi_{p q}(j)\right):(i, j) \in \mathfrak{g}\right\}$ is another digraph on $V$; we say that $\mathfrak{g}_{p q}$ is obtained from $\mathfrak{g}$ by interchanging $p$ and $q$. Observe that $o\left(p, \mathfrak{g}_{p q}\right)=o(q, \mathfrak{g})$, $o\left(q, \mathfrak{g}_{p q}\right)=o(p, \mathfrak{g})$, and $o\left(r, \mathfrak{g}_{p q}\right)=o(r, \mathfrak{g})$ for any $r$ other than $p$ or $q$. Evidently $\mathfrak{g}$ is obtained from $\mathfrak{g}_{p q}$ by interchanging $p$ and $q$.

Suppose that $\mathfrak{g}, p$, and $q$ satisfy the following condition: for each $r \in V \backslash\{p, q\}$, the digraph $\mathfrak{g}$ has an arc between $p$ and $r$ if and only if it has an arc between $q$ and $r$. (This condition holds for all $p, q \in V$ if $\mathfrak{g}$ is complete.) Then the digraph obtained from $\mathfrak{g}$ by interchanging $p$ and $q$ can also be obtained from $\mathfrak{g}$ by reversing arcs. Let $R$ consist of all pairs of arcs $\{(p, r),(r, q)\}$ or $\{(q, r),(r, p)\}$ such that both arcs are in $\mathfrak{g}$, plus the arc between $p$ and $q$ if there is one in $\mathfrak{g}$. Then $\mathfrak{g}_{p q}$ is obtained from $\mathfrak{g}$ by reversing the arcs in $R$. Recall the digraphs of Figure 3.1 . We find that reversing the arcs $(2,3),(3,4)$, and $(4,2)$ has the same effect on $\mathfrak{g}_{2}$ as interchanging 2 and 3 . On the other hand, there is no set of arcs in $\mathfrak{g}_{1}$ whose reversal gives us the digraph we obtain from $\mathfrak{g}_{1}$ by interchanging $b$ and $c$.

\section{Proof of Lemma 4.1}

Given a set $X$, on whose elements a weight function $w$ is defined, one way to prove that $\sum_{x \in X} w(x)=0$ is by defining a function $\phi: X \rightarrow X$ such that $w(\phi(x))=-w(x)$ and $\phi(\phi(x))=x$ for all $x \in X$. We say that $\phi$ is a weightpreserving and sign-reversing involution on $X$ if it has these properties. In effect, $\phi$ "pairs off" each $x$ with $\phi(x)$.

We shall prove Lemma 4.1 by constructing weight-preserving and sign-reversing involutions $\hat{\varphi}, \tilde{\varphi}$, and $\breve{\varphi}$ on the sets $\mathfrak{B}_{n} \backslash \mathfrak{B}_{n}^{*}, \mathfrak{C}_{n} \backslash \mathfrak{C}_{n}^{*}$, and $\mathfrak{C}_{n} \backslash \mathfrak{C}_{n}^{* *}$ respectively. The construction will involve arc reversal and will proceed in several phases. In the first two phases, the arcs to be reversed will all be of the form $(i, j)$ and $(-j,-i)$, where $|i|$ and $|j|$ are distinct and nonzero. This will allow $\hat{\varphi}, \tilde{\varphi}$, and $\breve{\varphi}$ to be defined simultaneously, because $\hat{w}(i, j)=\tilde{w}(i, j)=\breve{w}(i, j)$ whenever $j$ is not 0 or $-i$. In later phases, the functions will be defined separately. In Phases 1 and 3, we shall assume a total order has been defined on the set $\left(\begin{array}{c}{[n]} \\ 2\end{array}\right)$. 


\section{PHASE 1}

This phase takes care of all those $\mathfrak{g} \in \mathfrak{B}_{n} \cup \mathfrak{C}_{n}$ for which $\mathfrak{g}^{+}$is not transitive.

If $\mathfrak{g}^{+}$is not transitive, then we have $o\left(i, \mathfrak{g}^{+}\right)=o\left(j, \mathfrak{g}^{+}\right)$for some $(i, j) \in\left(\begin{array}{c}{[n]} \\ 2\end{array}\right)$. Let $\left(i_{0}, j_{0}\right)$ be the smallest $(i, j)$ (relative to the total order on $\left.\left(\begin{array}{c}{[n]} \\ 2\end{array}\right)\right)$ with this property. Let $\mathfrak{g}^{-}$be the negative subtournament of $\mathfrak{g}$, i.e., $\mathfrak{g}^{-}=\left\{(-j,-i):(i, j) \in \mathfrak{g}^{+}\right\}$. We obtain $\hat{\varphi}(\mathfrak{g}), \tilde{\varphi}(\mathfrak{g})$, or $\check{\varphi}(\mathfrak{g})$ by interchanging $i_{0}$ and $j_{0}$ in $\mathfrak{g}^{+}$and interchanging $-i_{0}$ and $-j_{0}$ in $\mathfrak{g}^{-}$. This corresponds to reversing the arc between $i_{0}$ and $j_{0}$, the arc between $-i_{0}$ and $-j_{0}$, and all the arcs that belong to subsets of $\mathfrak{g}$ of the forms

$$
\left\{\left(i_{0}, k\right),\left(k, j_{0}\right),\left(-j_{0},-k\right),\left(-k,-i_{0}\right)\right\}, \quad k>0
$$

and

$$
\left\{\left(j_{0}, k\right),\left(k, i_{0}\right),\left(-i_{0},-k\right),\left(-k,-j_{0}\right)\right\}, \quad k>0 .
$$

Let $\mathfrak{g}^{\prime}$ denote the result of interchanging $i_{0}$ and $j_{0}$ in $\mathfrak{g}^{+}$and $-i_{0}$ and $-j_{0}$ in $\mathfrak{g}^{-}$. We find that $\left(\mathfrak{g}^{\prime}\right)^{+}$is not transitive and that $o\left(k,\left(\mathfrak{g}^{\prime}\right)^{+}\right)=o\left(k, \mathfrak{g}^{+}\right)$for all $k \in[n]$, so we interchange the same vertices when applying $\hat{\varphi}, \tilde{\varphi}$, or $\check{\varphi}$ to $\mathfrak{g}^{\prime}$ as we had interchanged to obtain $\mathfrak{g}^{\prime}$ from $\mathfrak{g}$. This means that we recover $\mathfrak{g}$ from $\mathfrak{g}^{\prime}$ by applying the same function that gave us $\mathfrak{g}^{\prime}$ when applied to $\mathfrak{g}$.

We must now show that the weight of $\mathfrak{g}^{\prime}$ is -1 times the weight of $\mathfrak{g}$. Let $a$ and $b$ be the number of subsets of $\mathfrak{g}$ of types (a) and (b) respectively. We have

$$
0=o\left(i_{0}, \mathfrak{g}^{+}\right)-o\left(j_{0}, \mathfrak{g}^{+}\right)=a-b+ \begin{cases}1 & \text { if }\left(i_{0}, j_{0}\right) \in \mathfrak{g} \\ -1 & \text { if }\left(j_{0}, i_{0}\right) \in \mathfrak{g}\end{cases}
$$

Observe that reversing the arcs in a subset of type (a) multiplies the weight of $\mathfrak{g}$ by $x_{j_{0}} / x_{i_{0}}$, while reversing the arcs in a subset of type (b) multiplies the weight by $x_{i_{0}} / x_{j_{0}}$. If $\left(i_{0}, j_{0}\right) \in \mathfrak{g}$, then there is one more subset of type (b) than of type (a), so reversing the arcs in all subsets of both types multiplies the weight by $x_{i_{0}} / x_{j_{0}}$. Reversing $\left(-j_{0},-i_{0}\right)$ has no effect on the weight, whereas reversing $\left(i_{0}, j_{0}\right)$ multiplies the weight by $-x_{j_{0}} / x_{i_{0}}$. So the weight of $\mathfrak{g}^{\prime}$ is -1 times the weight of $\mathfrak{g}$ as required. Similarly if $\left(j_{0}, i_{0}\right) \in \mathfrak{g}$.

This completes Phase 1 . From now on, we shall work only with digraphs whose positive subtournaments are transitive. Recall the definitions of $\sigma(\mathfrak{g}), \tau(\mathfrak{g}), \bar{\tau}(\mathfrak{g})$, $\omega_{0}(\mathfrak{g})$, and $\omega_{ \pm}(\mathfrak{g})$ for digraphs $\mathfrak{g}$ with $\mathfrak{g}^{+}$transitive.

\section{Phase 2}

In this phase, we define $\hat{\varphi}, \tilde{\varphi}$, and $\breve{\varphi}$ for all $\mathfrak{g}$ such that $\mathfrak{g}^{+}$is transitive but $\tau(\mathfrak{g})$ is not an order ideal of $\left(\begin{array}{c}{[n]} \\ 2\end{array}\right)$. We begin with a discussion of how reversing a certain set of arcs in $\mathfrak{g}$ affects $\tau(\mathfrak{g})$ and $\bar{\tau}(\mathfrak{g})$.

Suppose $T$ and $T^{\prime}$ are subsets of a set $A$. If there exist disjoint subsets of $A$ of the form $\left\{a_{i}: i \in I\right\}$ and $\left\{a_{i}^{\prime}: i \in I\right\}$ such that for each $i \in I$, we have $a_{i} \in T$ if and only if $a_{i}^{\prime} \in T^{\prime}$ and $a_{i}^{\prime} \in T$ if and only if $a_{i} \in T^{\prime}$, then we say that $T^{\prime}$ is obtained from $T$ by exchanging the pairs $a_{i}$ and $a_{i}^{\prime}$ for each $i \in I$. For example, we 
obtain $\{1,4,5,6,10\}$ from $\{2,3,5,6,9\}$ by exchanging 1 and 2,3 and 4,5 and 6,7 and 8 , and 9 and 10 . If $\mathfrak{g}$ is a digraph on the set $V$ and $\mathfrak{g}_{p q}$ is obtained from $\mathfrak{g}$ by interchanging the vertices $p$ and $q$, then $\mathfrak{g}_{p q}$ is also obtained from $\mathfrak{g}$ by exchanging $(p, r)$ and $(q, r)$ for all $r \in V \backslash\{p, q\}$; exchanging $(r, p)$ and $(r, q)$ for all $r \in V \backslash\{p, q\}$; and exchanging $(p, q)$ and $(q, p)$. Evidently if $T^{\prime}$ is obtained from $T$ by exchanging $a_{i}$ and $a_{i}^{\prime}$ for each $i \in I$, then $T$ is obtained from $T^{\prime}$ by the same process.

Now let $\mathfrak{g} \in \mathfrak{B}_{n} \cup \mathfrak{C}_{n}$ with $\mathfrak{g}^{+}$transitive and $\sigma=\sigma(\mathfrak{g})$. Given $i, j \in[n]$ with $i<j$, we create a new digraph $\mathfrak{g}^{\prime}$ by reversing the arcs

$$
(\sigma(i), \sigma(k)),(\sigma(k), \sigma(j)),(-\sigma(k),-\sigma(i)),(-\sigma(j),-\sigma(k)), \quad i<k<j,
$$

and reversing $(\sigma(i), \sigma(j))$ and $(-\sigma(j),-\sigma(i))$. It is easy to see that $\left(\mathfrak{g}^{\prime}\right)^{+}$is transitive, with $\sigma\left(\mathfrak{g}^{\prime}\right)=\sigma \cdot(i j)$. We find that $\tau\left(\mathfrak{g}^{\prime}\right)$ and $\bar{\tau}\left(\mathfrak{g}^{\prime}\right)$ are generally not the same as $\tau(\mathfrak{g})$ and $\bar{\tau}(\mathfrak{g})$. This is because $\tau$ and $\bar{\tau}$ depend upon $\sigma$. Indeed, $\tau\left(\mathfrak{g}^{\prime}\right)$ and $\bar{\tau}\left(\mathfrak{g}^{\prime}\right)$ are obtained from $\tau(\mathfrak{g})$ and $\bar{\tau}(\mathfrak{g})$ by exchanging $(k, i)$ and $(k, j), 1 \leq k<i ;(i, k)$ and $(k, j), i<k<j ;(i, k)$ and $(j, k), j<k \leq n ;$ and $(i, i)$ and $(j, j)$. For instance, if $\mathfrak{g} \in \mathfrak{B}_{6} \cup \mathfrak{C}_{6}$ is such that $\tau(\mathfrak{g})$ is the set shown in Figure 5.3, with $i=2$ and $j=4$, then the pairs of elements of $\left(\begin{array}{c}66 \\ 2\end{array}\right)$ to be exchanged to obtain $\tau\left(\mathfrak{g}^{\prime}\right)$ are those connected by arrows in the figure. Observe that $\tau\left(\mathfrak{g}^{\prime}\right)=\tau(\mathfrak{g})$ exactly when $i$ and $j$ are interchangeable in $\tau(\mathfrak{g})$, and similarly for $\bar{\tau}$.

We now begin the second phase of the description of $\hat{\varphi}, \tilde{\varphi}$, and $\breve{\varphi}$. Given $\mathfrak{g} \in$ $\mathfrak{B}_{n} \cup \mathfrak{C}_{n}$ with $\tau=\tau(\mathfrak{g})$ not an order ideal of $\left(\begin{array}{c}{[n]} \\ 2\end{array}\right)$, we either have $(q, p) \notin \tau$, $(q, p+1) \in \tau$ for some $q<p$ or $(p, q) \notin \tau,(p+1, q) \in \tau$ for some $q>p+1$. In either case, $\mathfrak{g}$ contains a pair of cycles of the form

$$
\begin{gathered}
\{(\sigma(p), \sigma(p+1)),(\sigma(p+1),-\sigma(q)),(-\sigma(q), \sigma(p))\} \\
\text { and }\{(-\sigma(p), \sigma(q)),(\sigma(q),-\sigma(p+1)),(-\sigma(p+1),-\sigma(p))\} .
\end{gathered}
$$

We shall reverse some such pair of cycles (i.e., reverse all the arcs in the cycles) to obtain $\hat{\varphi}(\mathfrak{g}), \tilde{\varphi}(\mathfrak{g})$, or $\breve{\varphi}(\mathfrak{g})$. Let $\mathfrak{g}^{\prime}$ denote the result of this cycle reversal. It is easy to see that $\left(\mathfrak{g}^{\prime}\right)^{+}$is transitive, with corresponding permutation $\sigma\left(\mathfrak{g}^{\prime}\right)=\sigma \cdot(p p+1)$, and that $\hat{w}\left(\mathfrak{g}^{\prime}\right)=-\hat{w}(\mathfrak{g}), \tilde{w}\left(\mathfrak{g}^{\prime}\right)=-\tilde{w}(\mathfrak{g})$, and $\breve{w}\left(\mathfrak{g}^{\prime}\right)=-\breve{w}(\mathfrak{g})$. To ensure that we recover $\mathfrak{g}$ by applying $\hat{\varphi}, \tilde{\varphi}$, or $\check{\varphi}$ to $\mathfrak{g}^{\prime}$, we must have some way of choosing which cycles to reverse. This is the purpose of the following discussion.

We say there is a violation in the $i$ th row of $\tau$ if there is some $l$ such that $(i, l) \notin \tau$ and $(i, l+1) \in \tau$. We say the $k$ th row of $\tau$ extends farther than the $i$ th row if $\max \{j:(k, j) \in \tau\}>\max \{j:(i, j) \in \tau\}$. Find the smallest $i$ such that either there is a violation in the $i$ th row of $\tau$, or there is a $k>i$ such that the $k$ th row of $\tau$ extends farther than the $i$ th row. (Since $\tau$ is not an order ideal, there is an $i$ satisfying one of these two conditions.) Find the smallest $j$ such that $(k, l) \notin \tau$ whenever $k \geq i$ and $l>j$. Then we have $(k, l) \in \tau$ whenever $k<i$ and $l \leq j$, since there are no violations in the first $i-1$ rows and no row below the $(i-1)$ st extends farther than any of the first $i-1$ rows. For example, with $\tau$ as in Figure 6.1, we find that $i=2$ and $j=6$. 


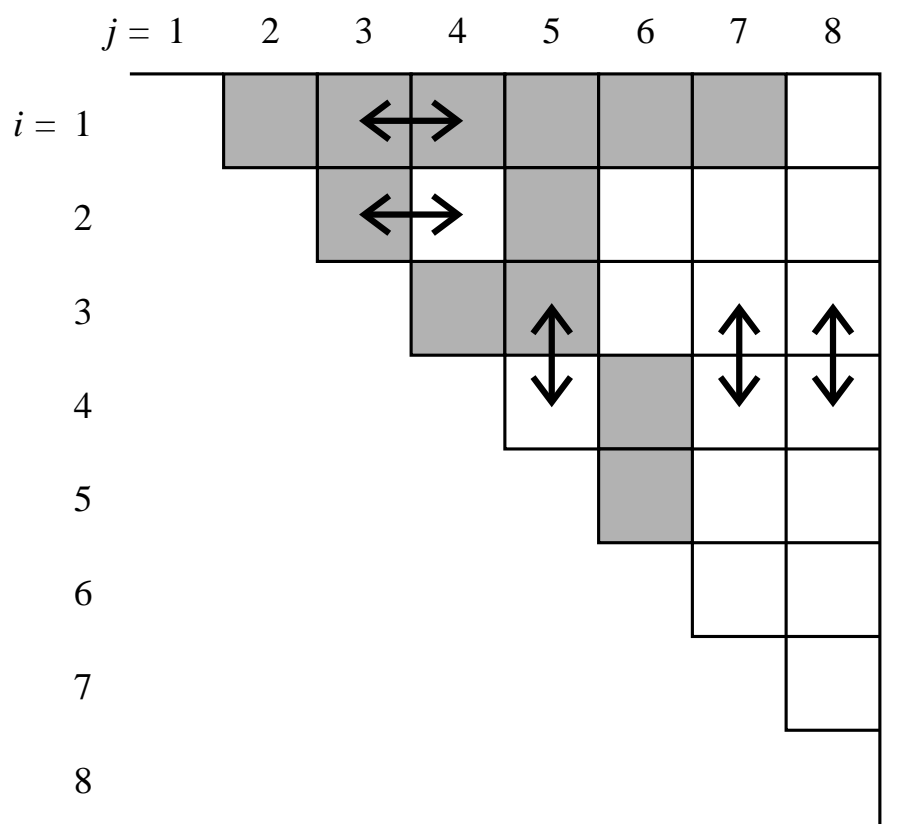

FigURE 6.1

Now either $(i, j)$ is in $\tau$, or it is not. If $(i, j) \notin \tau$, as is the case for $\tau$ as in Figure 6.1 , then there must be a row below the $i$ th that extends farther than the $i$ th row, since we found the smallest $j$ such that $(k, l) \notin \tau$ whenever $k \geq i$ and $l>j$. So there must be an $l$ with $i+1 \leq l \leq j-1,(l-1, j) \notin \tau$, and $(l, j) \in \tau$. Choose the smallest such $l$; then let $p=l-1$ and $q=j$ and reverse the cycles shown in ( $\dagger$ ). If $\mathfrak{g}^{\prime}$ is the digraph that results from these cycle reversals, then $\sigma\left(\mathfrak{g}^{\prime}\right)=\sigma \cdot(l-1 l)$ and $\tau^{\prime}=\tau\left(\mathfrak{g}^{\prime}\right)$ is obtained from $\tau$ by exchanging $(k, l-1)$ and $(k, l)$ for $1 \leq k \leq l-2$ and exchanging $(l-1, k)$ and $(l, k)$ for $l+1 \leq k \leq n, k \neq j$. In the situation of Figure 6.1 , we have $l=4$, and the arrows in the figure indicate the exchanges by which $\tau^{\prime}$ is obtained from $\tau$. Observe that the $j$ th column of $\tau^{\prime}$ is the same as that of $\tau ;(k, l) \notin \tau^{\prime}$ whenever $k \geq i$ and $l>j$; and $(k, l) \in \tau^{\prime}$ whenever $k<i$ and $l \leq j$. This means that when we examine $\tau^{\prime}$ to choose which cycles in $\mathfrak{g}^{\prime}$ to reverse, we shall find the same $i$ and $j$ and the same $l$ as we had found for $\tau$. So we recover $\mathfrak{g}$ by applying $\hat{\varphi}, \tilde{\varphi}$, or $\breve{\varphi}$ to $\mathfrak{g}^{\prime}$, as required.

If $(i, j) \in \tau$, then no row below the $i$ th extends farther than the $i$ th row, so there must be a violation in the $i$ th row of $\tau$; we must have some $k$ with $i+1 \leq k \leq j-1$ such that $(i, k) \notin \tau$ and $(i, k+1) \in \tau$. Choose the largest such $k$. Let $p=k$ and $q=i$ and reverse the cycles shown in ( $\dagger$ ). If $\mathfrak{g}^{\prime}$ is the digraph that results from these reversals, then we have $\sigma\left(\mathfrak{g}^{\prime}\right)=\sigma \cdot(k k+1)$ and $\tau^{\prime}=\tau\left(\mathfrak{g}^{\prime}\right)$ is obtained from $\tau$ by exchanging $(l, k)$ and $(l, k+1)$ for $1 \leq l \leq k-1, l \neq i$, and exchanging $(k, l)$ and $(k+1, l)$ for $k+2 \leq l \leq n$. We find that the $i$ th row of $\tau^{\prime}$ is the same as that of $\tau$, and that $(k, l)$ is in $\tau$ whenever $k<i$ and $l \leq j$ and not in $\tau$ whenever $k \geq i$ and $l>j$. So when we look at $\tau^{\prime}$ to decide which cycles in $\mathfrak{g}^{\prime}$ to reverse, we shall 
find the same $i, j$, and $k$ as we had found for $\tau$. Again, we recover $\mathfrak{g}$ from $\mathfrak{g}^{\prime}$ by applying $\hat{\varphi}, \tilde{\varphi}$, or $\check{\varphi}$. We are finished with Phase 2 .

\section{Phase 3}

From now on, we assume $\mathfrak{g}^{+}$is transitive and $\tau=\tau(\mathfrak{g})$ is an order ideal of $\left(\begin{array}{c}{[n]} \\ 2\end{array}\right)$. Lemma 5.1(a) implies that $\#(1, \tau) \geq \#(2, \tau) \geq \cdots \geq \#(n, \tau)$. If $\mathfrak{g} \in \mathfrak{B}_{n}$, then we have

$$
o(\sigma(i), \mathfrak{g})=n-i+\#(i, \tau)+\chi\left(i \in \omega_{0}\right)
$$

for all $i \in[n]$; if $\mathfrak{g} \in \mathfrak{C}_{n}$, then

$$
\begin{aligned}
o(\sigma(i), \mathfrak{g}) & =n-i+\#(i, \tau)+\chi\left(i \in \omega_{0}\right)+\chi\left(i \in \omega_{ \pm}\right) \\
& =n-i+\#_{0}(i, \bar{\tau})+\chi\left(i \in \omega_{ \pm}\right)
\end{aligned}
$$

for all $i \in[n]$.

Construction of $\hat{\varphi}$. Given $\mathfrak{g} \in \mathfrak{B}_{n}$, with $\mathfrak{g}^{+}$transitive and $\tau$ an order ideal of $\left(\begin{array}{c}{[n]} \\ 2\end{array}\right)$, we have $\mathfrak{g} \in \mathfrak{B}_{n}^{*}$ unless $o(\sigma(i), \mathfrak{g}) \leq o(\sigma(i+1), \mathfrak{g})$ for some $i$. Since $\#(1, \tau) \geq$ $\#(2, \tau) \geq \cdots \geq \#(n, \tau)$, the only way we can have $o(\sigma(i), \mathfrak{g}) \leq o(\sigma(i+1), \mathfrak{g})$ is if $\#(i, \tau)=\#(i+1, \tau), i \notin \omega_{0}$, and $i+1 \in \omega_{0}$; this gives us $o(\sigma(i), \mathfrak{g})=o(\sigma(i+1), \mathfrak{g})$. Let $i_{0}$ be the smallest $i$ with these properties. We obtain $\hat{\varphi}(\mathfrak{g})$ from $\mathfrak{g}$ by reversing the pair of cycles

$$
\begin{gathered}
\left\{\left(\sigma\left(i_{0}\right), \sigma\left(i_{0}+1\right)\right),\left(\sigma\left(i_{0}+1\right), 0\right),\left(0, \sigma\left(i_{0}\right)\right)\right\} \\
\text { and }\left\{\left(-\sigma\left(i_{0}\right), 0\right),\left(0,-\sigma\left(i_{0}+1\right)\right),\left(-\sigma\left(i_{0}+1\right),-\sigma\left(i_{0}\right)\right)\right\}
\end{gathered}
$$

We find that $\hat{\varphi}(\mathfrak{g})^{+}$is transitive, with $\sigma(\hat{\varphi}(\mathfrak{g}))=\sigma \cdot\left(i_{0} i_{0}+1\right)$, and that $\hat{w}(\hat{\varphi}(\mathfrak{g}))=$ $-\hat{w}(\mathfrak{g})$. Lemma 5.2(a) tells us that $i_{0}$ and $i_{0}+1$ are interchangeable in $\tau$; therefore $\tau(\hat{\varphi}(\mathfrak{g}))=\tau$. Finally, we observe that $\omega_{0}(\hat{\varphi}(\mathfrak{g}))=\omega_{0}(\mathfrak{g})$. We conclude that $\hat{\varphi}(\hat{\varphi}(\mathfrak{g}))=\mathfrak{g}$, as required. This completes the proof that $\sum_{\mathfrak{g} \in \mathfrak{B}_{n} \backslash \mathfrak{B}_{n}^{*}} \hat{w}(\mathfrak{g})=0$.

Construction of $\tilde{\varphi}$. Suppose $\mathfrak{g}$ is in $\mathfrak{C}_{n}$, with $\mathfrak{g}^{+}$transitive, $\tau$ an order ideal of $\left(\begin{array}{c}{[n]} \\ 2\end{array}\right)$, but $\bar{\tau}$ not an order ideal of $\left\langle\begin{array}{c}{[n]} \\ 2\end{array}\right)$. For any $\bar{T} \subseteq\left\langle\begin{array}{c}{[n]} \\ 2\end{array}\right)$ such that $T=\bar{T} \cap\left(\begin{array}{c}{[n]} \\ 2\end{array}\right)$ is an order ideal of $\left(\begin{array}{c}{[n]} \\ 2\end{array}\right)$, let $X(\bar{T})$ be the set of all $(i, j) \in\left(\begin{array}{c}{[n]} \\ 2\end{array}\right)$ such that either

$$
(i, j) \in I(T) \quad \text { and } \quad(i, i),(j, j) \notin \bar{T}
$$

or

$$
(i, j) \in O(T) \text { and }(i, i),(j, j) \in \bar{T} \text {. }
$$

In Figure 6.2, for example, we have a subset $\bar{\tau}$ of $\left\langle\begin{array}{c}{[6]} \\ 2\end{array}\right\rangle$ for which $X(\bar{\tau})=\{(2,5)\}$. 


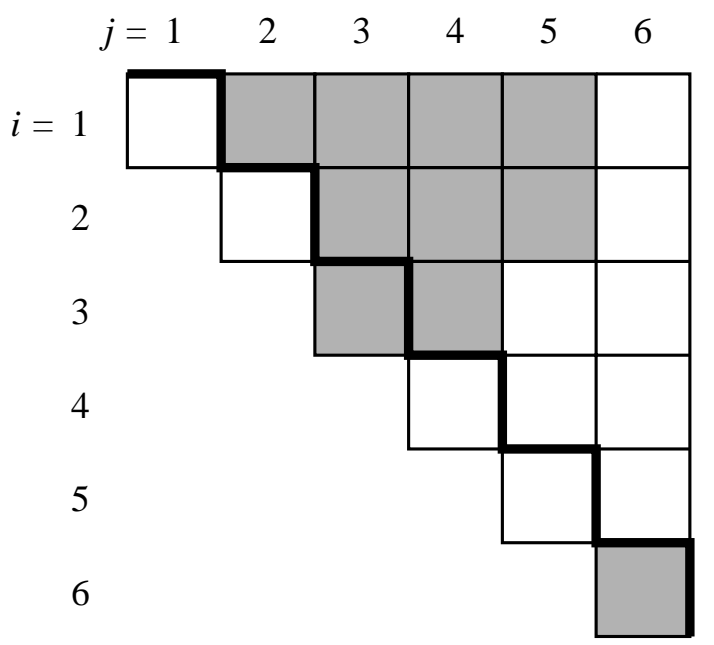

FiguRe 6.2

If $X(\bar{\tau}) \neq \emptyset$, then let $\left(i_{0}, j_{0}\right)$ be the smallest element of $X(\bar{\tau})$ relative to our total order on $\left(\begin{array}{c}{[n]} \\ 2\end{array}\right)$. If $\left(i_{0}, j_{0}\right) \in I(\tau)$, then we obtain $\tilde{\varphi}(\mathfrak{g})$ from $\mathfrak{g}$ by reversing the cycles

$$
\begin{array}{r}
\left\{\left(\sigma\left(i_{0}\right),-\sigma\left(j_{0}\right)\right),\left(-\sigma\left(j_{0}\right), 0\right),\left(0, \sigma\left(i_{0}\right)\right)\right\} \\
\text { and }\left\{\left(-\sigma\left(i_{0}\right), 0\right),\left(0, \sigma\left(j_{0}\right)\right),\left(\sigma\left(j_{0}\right),-\sigma\left(i_{0}\right)\right)\right\}
\end{array}
$$

Otherwise, $\left(i_{0}, j_{0}\right) \in O(\tau)$, in which case we obtain $\tilde{\varphi}(\mathfrak{g})$ by reversing

$$
\begin{array}{r}
\left\{\left(\sigma\left(i_{0}\right), 0\right),\left(0,-\sigma\left(j_{0}\right)\right),\left(-\sigma\left(j_{0}\right), \sigma\left(i_{0}\right)\right)\right\} \\
\text { and }\left\{\left(-\sigma\left(i_{0}\right), \sigma\left(j_{0}\right)\right),\left(\sigma\left(j_{0}\right), 0\right),\left(0,-\sigma\left(i_{0}\right)\right)\right\} .
\end{array}
$$

It is easy to see that $\tilde{w}(\tilde{\varphi}(\mathfrak{g}))=-\tilde{w}(\mathfrak{g})$. Observe that $\tilde{\varphi}(\mathfrak{g})^{+}=\mathfrak{g}^{+}$, so $\tilde{\varphi}(\mathfrak{g})^{+}$is transitive. We also have $\omega_{ \pm}(\tilde{\varphi}(\mathfrak{g}))=\omega_{ \pm}(\mathfrak{g})$.

Let $\tau^{\prime}=\tau(\tilde{\varphi}(\mathfrak{g}))$ and $\bar{\tau}^{\prime}=\bar{\tau}(\tilde{\varphi}(\mathfrak{g}))$. We see that if $\left(i_{0}, j_{0}\right) \in I(\tau)$ (respectively, $O(\tau)$ ), then $\left(i_{0}, j_{0}\right) \in O\left(\tau^{\prime}\right)$ (respectively, $\left.I\left(\tau^{\prime}\right)\right)$. If $k$ is not $i_{0}$ or $j_{0}$, then $(k, k) \in \bar{\tau}^{\prime}$ if and only if $(k, k) \in \bar{\tau}$, whereas $\left(i_{0}, i_{0}\right)$ and $\left(j_{0}, j_{0}\right)$ are in $\bar{\tau}^{\prime}$ if and only if they are not in $\bar{\tau}$. This tells us that $\left(i_{0}, j_{0}\right) \in X\left(\bar{\tau}^{\prime}\right)$; we shall now prove that $X\left(\bar{\tau}^{\prime}\right)=X(\bar{\tau})$, which implies that $\left(i_{0}, j_{0}\right)$ is the smallest element in $X\left(\bar{\tau}^{\prime}\right)$. This in turn means that $\tilde{\varphi}(\tilde{\varphi}(\mathfrak{g}))=\mathfrak{g}$ if $X(\bar{\tau})$ is nonempty.

Suppose $(i, j) \in X\left(\bar{\tau}^{\prime}\right) \backslash X(\bar{\tau})$. Either $(i, j) \in I\left(\tau^{\prime}\right)$ or $(i, j) \in O\left(\tau^{\prime}\right)$. Assuming the former, we have $(i, i),(j, j) \notin \bar{\tau}^{\prime}$. If $i \neq i_{0}$ and $j \neq j_{0}$, then $(i, i),(j, j) \notin \bar{\tau}$. In this case, we must have $(i, j) \notin I(\tau)$ in order to have $(i, j) \notin X(\bar{\tau})$. For this to happen with $(i, j)$ in $I\left(\tau^{\prime}\right)$, one of $(i+1, j),(i, j+1)$ must be in $\tau$ and must be removed from $\tau$ to yield $\tau^{\prime}$. Since $\tau^{\prime}$ is either $\tau \cup\left\{\left(i_{0}, j_{0}\right)\right\}$ or $\tau \backslash\left\{\left(i_{0}, j_{0}\right)\right\}$, it must be that $\left(i_{0}, j_{0}\right)$ equals $(i+1, j)$ or $(i, j+1)$. This contradicts our assumption that $i \neq i_{0}$ and $j \neq j_{0}$. So assume that $i=i_{0}$ or $j=j_{0}$. Then either $\left(i_{0}, i_{0}\right) \notin \bar{\tau}^{\prime}$ or $\left(j_{0}, j_{0}\right) \notin \bar{\tau}^{\prime}$, by our assumption that $(i, j) \in I\left(\tau^{\prime}\right)$. But $\left(i_{0}, j_{0}\right) \in X\left(\bar{\tau}^{\prime}\right)$, so $\left(i_{0}, i_{0}\right) \notin \bar{\tau}^{\prime}$ if and only if $\left(j_{0}, j_{0}\right) \notin \bar{\tau}^{\prime}$ if and only if $\left(i_{0}, j_{0}\right) \in I\left(\tau^{\prime}\right)$. Since no row or 
column of $\left(\begin{array}{c}{[n]} \\ 2\end{array}\right)$ can contain two distinct inner extreme points of $\tau^{\prime}$, our assumption that $i=i_{0}$ or $j=j_{0}$ now tells us that $(i, j)=\left(i_{0}, j_{0}\right)$, which contradicts our assumption that $(i, j) \notin X(\bar{\tau})$. The argument is similar if we begin by assuming $(i, j) \in O\left(\tau^{\prime}\right)$. We conclude that $X\left(\bar{\tau}^{\prime}\right) \subseteq X(\bar{\tau})$; similarly, $X(\bar{\tau}) \subseteq X\left(\bar{\tau}^{\prime}\right)$.

There is another possibility to be considered if $\tau$ is an order ideal of $\left(\begin{array}{c}{[n]} \\ 2\end{array}\right)$ but $\bar{\tau}$ is not an order ideal of $\left\langle{ }_{2}^{[n]}{ }_{2}\right\rangle$ : that $X(\bar{\tau})$ is empty (this is the case for $\bar{\tau}$ as in Figure $5.5)$. In this case, Lemma 5.3 tells us that there is some $i \in[n]$ such that $(i, i) \notin \bar{\tau}$, $(i+1, i+1) \in \bar{\tau}$, and $i$ and $i+1$ are interchangeable in $\tau$. Let $i_{0}$ be the smallest such $i$. Then we obtain $\tilde{\varphi}(\mathfrak{g})$ from $\mathfrak{g}$ by reversing the cycles

$$
\begin{gathered}
\left\{\left(\sigma\left(i_{0}\right), \sigma\left(i_{0}+1\right)\right),\left(\sigma\left(i_{0}+1\right), 0\right),\left(0, \sigma\left(i_{0}\right)\right)\right\} \\
\text { and }\left\{\left(-\sigma\left(i_{0}\right), 0\right),\left(0,-\sigma\left(i_{0}+1\right)\right),\left(-\sigma\left(i_{0}+1\right),-\sigma\left(i_{0}\right)\right)\right\}
\end{gathered}
$$

We see that $\tilde{\varphi}(\mathfrak{g})^{+}$is transitive, with $\sigma(\tilde{\varphi}(\mathfrak{g}))=\sigma \cdot\left(i_{0} i_{0}+1\right)$, and that $\tilde{w}(\tilde{\varphi}(\mathfrak{g}))=$ $-\tilde{w}(\mathfrak{g})$. We obtain $\omega_{ \pm}(\tilde{\varphi}(\mathfrak{g}))$ from $\omega_{ \pm}(\mathfrak{g})$ by exchanging $i_{0}$ and $i_{0}+1$, but $\omega_{0}(\tilde{\varphi}(\mathfrak{g}))=$ $\omega_{0}(\mathfrak{g})$. Meanwhile, $\tau(\tilde{\varphi}(\mathfrak{g}))=\tau$, due to the interchangeability of $i_{0}$ and $i_{0}+1$ in $\tau$. So $\bar{\tau}(\tilde{\varphi}(\mathfrak{g}))=\bar{\tau}$, and this ensures that we shall obtain $\mathfrak{g}$ by applying $\tilde{\varphi}$ to $\tilde{\varphi}(\mathfrak{g})$.

Construction of $\check{\varphi}$. Let $\mathfrak{g} \in \mathfrak{C}_{n}$ with $\mathfrak{g}^{+}$transitive, $\tau$ an order ideal of $\left(\begin{array}{c}{[n]} \\ 2\end{array}\right)$, and $o(\sigma(i), \mathfrak{g})=o(\sigma(j), \mathfrak{g})$ for some $i, j \in[n]$ with $i<j$. Let $i_{0}$ be the smallest $i$ for which this occurs. Then $j$ must be either $i_{0}+1$ or $i_{0}+2$, since we have $o(\sigma(k), \mathfrak{g}) \leq \#(k, \tau)+n-k+2<\#(k, \tau)+n-i_{0} \leq \#\left(i_{0}, \tau\right)+n-i_{0} \leq o\left(\sigma\left(i_{0}\right), \mathfrak{g}\right)$ whenever $k>i_{0}+2$.

Suppose $o\left(\sigma\left(i_{0}\right), \mathfrak{g}\right)=o\left(\sigma\left(i_{0}+1\right), \mathfrak{g}\right)$. There are several ways in which this can occur:

(1) \#( $\left.i_{0}, \tau\right)=\#\left(i_{0}+1, \tau\right), i_{0} \notin \omega_{0}, i_{0}+1 \in \omega_{0}$, and $i_{0} \in \omega_{ \pm}$if and only if $i_{0}+1 \in \omega_{ \pm}$.

(2) \#( $\left.i_{0}, \tau\right)=\#\left(i_{0}+1, \tau\right), i_{0} \notin \omega_{ \pm}, i_{0}+1 \in \omega_{ \pm}$, and $i_{0} \in \omega_{0}$ if and only if $i_{0}+1 \in \omega_{0}$.

(3) \# $\left(i_{0}, \tau\right)=\#\left(i_{0}+1, \tau\right)+1, i_{0} \notin \omega_{0}, i_{0} \notin \omega_{ \pm}, i_{0}+1 \in \omega_{0}, i_{0}+1 \in \omega_{ \pm}$.

For $(1)$, we obtain $\breve{\varphi}(\mathfrak{g})$ from $\mathfrak{g}$ by reversing the cycles

$$
\begin{gathered}
\left\{\left(\sigma\left(i_{0}\right), \sigma\left(i_{0}+1\right)\right),\left(\sigma\left(i_{0}+1\right), 0\right),\left(0, \sigma\left(i_{0}\right)\right)\right\} \\
\text { and }\left\{\left(-\sigma\left(i_{0}\right), 0\right),\left(0,-\sigma\left(i_{0}+1\right)\right),\left(-\sigma\left(i_{0}+1\right),-\sigma\left(i_{0}\right)\right)\right\}
\end{gathered}
$$

We observe that $\breve{\varphi}(\mathfrak{g})^{+}$is transitive, with corresponding permutation $\sigma \cdot\left(i_{0} i_{0}+1\right)$. Meanwhile, $\omega_{0}(\check{\varphi}(\mathfrak{g}))=\omega_{0}$ and $\omega_{ \pm}(\check{\varphi}(\mathfrak{g}))=\omega_{ \pm}$. Since $\#\left(i_{0}, \tau\right)=\#\left(i_{0}+1, \tau\right)$ in this case, $i_{0}$ and $i_{0}+1$ are interchangeable in $\tau$ and we have $\tau(\breve{\varphi}(\mathfrak{g}))=\tau$. From these observations, we conclude that $\breve{\varphi}(\breve{\varphi}(\mathfrak{g}))=\mathfrak{g}$. And it is easy to see that $\breve{w}(\breve{\varphi}(\mathfrak{g}))=-\breve{w}(\mathfrak{g})$.

(2) is very similar to $(1)$. We obtain $\breve{\varphi}(\mathfrak{g})$ by reversing the cycle

$$
\left\{\left(\sigma\left(i_{0}\right), \sigma\left(i_{0}+1\right)\right),\left(\sigma\left(i_{0}+1\right),-\sigma\left(i_{0}+1\right)\right),\left(-\sigma\left(i_{0}+1\right),-\sigma\left(i_{0}\right)\right),\left(-\sigma\left(i_{0}\right), \sigma\left(i_{0}\right)\right)\right\} .
$$


The observations in (1) apply in this case, and we have $\breve{\varphi}(\breve{\varphi}(\mathfrak{g}))=\mathfrak{g}$ and $\breve{w}(\breve{\varphi}(\mathfrak{g}))=$ $-\breve{w}(\mathfrak{g})$.

(3) is a little more difficult. Suppose that $\#\left(i_{0}, \tau\right)=\#\left(i_{0}+1, \tau\right)+1=r$. Observe that $r \neq i_{0}$ : it is not hard to see that if $\tau$ is an order ideal of $\left(\begin{array}{c}{[n]} \\ 2\end{array}\right)$ and $\#(i, \tau)=i$ for some $i$, then $\#(i+1, \tau)=i$ as well. If $r<i_{0}$, then we have

$$
\begin{aligned}
\left(1, i_{0}\right),\left(2, i_{0}\right), \ldots,\left(r-1, i_{0}\right),\left(r, i_{0}\right) & \in \tau, \\
\left(1, i_{0}+1\right),\left(2, i_{0}+1\right), \ldots,\left(r-1, i_{0}+1\right) & \in \tau, \quad\left(r, i_{0}+1\right) \notin \tau,
\end{aligned}
$$

and

$$
\left(k, i_{0}\right),\left(k, i_{0}+1\right) \notin \tau \text { for } r<k<i, \quad\left(i_{0}, k\right),\left(i_{0}+1, k\right) \notin \tau \text { for } i_{0}+1<k<n .
$$

The only thing preventing $i_{0}$ and $i_{0}+1$ from being interchangeable in $\tau$ is that $\left(r, i_{0}\right) \in \tau$ but $\left(r, i_{0}+1\right) \notin \tau$. So if we reverse the arcs $\left(\sigma\left(i_{0}\right), \sigma\left(i_{0}+1\right)\right)$ and $\left(-\sigma\left(i_{0}+1\right),-\sigma\left(i_{0}\right)\right)$, we must also reverse the arcs connecting $\pm \sigma(r)$ to $\mp \sigma\left(i_{0}\right)$ and to $\mp \sigma\left(i_{0}+1\right)$ in order to preserve $\tau$. This is part of what we do to obtain $\breve{\varphi}(\mathfrak{g})$. In addition, we reverse the arcs connecting $0, \sigma\left(i_{0}\right)$, and $-\sigma\left(i_{0}\right)$ and the arcs connecting $0, \sigma\left(i_{0}+1\right)$, and $-\sigma\left(i_{0}+1\right)$. The set of all these arcs may be written as a disjoint union of cycles:

and

$$
\begin{aligned}
& \left\{\left(\sigma\left(i_{0}\right), \sigma\left(i_{0}+1\right)\right),\left(\sigma\left(i_{0}+1\right), 0\right),\left(0,-\sigma\left(i_{0}+1\right)\right)\right\}, \\
& \left\{\left(-\sigma\left(i_{0}+1\right),-\sigma\left(i_{0}\right)\right),\left(-\sigma\left(i_{0}\right), 0\right),\left(0, \sigma\left(i_{0}\right)\right)\right\}
\end{aligned}
$$

$$
\begin{gathered}
\left\{\left(\sigma\left(i_{0}\right),-\sigma(r)\right),\left(-\sigma(r), \sigma\left(i_{0}+1\right)\right),\left(\sigma\left(i_{0}+1\right),-\sigma\left(i_{0}+1\right)\right)\right. \\
\left.\left(-\sigma\left(i_{0}+1\right), \sigma(r)\right),\left(\sigma(r),-\sigma\left(i_{0}\right)\right),\left(-\sigma\left(i_{0}\right), \sigma\left(i_{0}\right)\right)\right\} .
\end{gathered}
$$

If $r>i_{0}$, then the only thing preventing $i_{0}$ and $i_{0}+1$ from being interchangeable in $\tau$ is that $\left(i_{0}, r+1\right) \in \tau$ but $\left(i_{0}+1, r+1\right) \notin \tau$. The cycles we reverse to obtain $\breve{\varphi}(\mathfrak{g})$ are those shown above, except that $r$ is replaced with $r+1$.

The digraph $\mathfrak{g} \in \mathfrak{C}_{3}$ portrayed in Figure 6.3 gives an example of the situation in case (3). We have $\sigma(2)=1, \sigma(3)=3$, and $o(1, \mathfrak{g})=o(3, \mathfrak{g})=2$. We obtain $\breve{\varphi}(\mathfrak{g})$ by reversing the bold arcs, which contribute $t x_{1}^{2} x_{2} x_{3}^{2}$ to $\breve{w}(\mathfrak{g})$. 


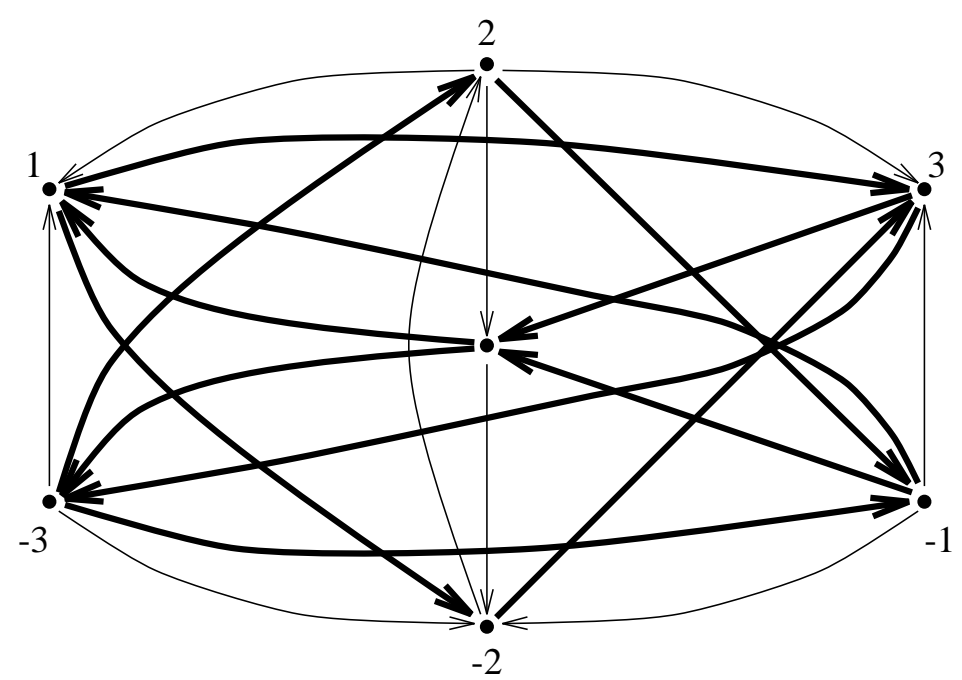

FiguRE 6.3

We find that $\breve{\varphi}(\mathfrak{g})^{+}$is transitive, corresponding to $\sigma \cdot\left(i_{0} i_{0}+1\right)$; meanwhile, $\tau(\check{\varphi}(\mathfrak{g}))=\tau, \omega_{0}(\breve{\varphi}(\mathfrak{g}))=\omega_{0}$, and $\omega_{ \pm}(\breve{\varphi}(\mathfrak{g}))=\omega_{ \pm}$. So once again, we have $\breve{\varphi}(\breve{\varphi}(\mathfrak{g}))=$ $\mathfrak{g}$ and $\breve{w}(\breve{\varphi}(\mathfrak{g}))=-\breve{w}(\mathfrak{g})$. This completes the description of $\check{\varphi}$ in case $o\left(\sigma\left(i_{0}\right), \mathfrak{g}\right)=$ $o\left(\sigma\left(i_{0}+1\right), \mathfrak{g}\right)$.

Now suppose $o\left(\sigma\left(i_{0}\right), \mathfrak{g}\right)=o\left(\sigma\left(i_{0}+2\right), \mathfrak{g}\right)$. There is only one way this can happen: $\#\left(i_{0}, \tau\right)=\#\left(i_{0}+1, \tau\right)=\#\left(i_{0}+2, \tau\right)$ and $i_{0} \notin \omega_{0}, i_{0} \notin \omega_{ \pm}, i_{0}+2 \in \omega_{0}, i_{0}+2 \in \omega_{ \pm}$. To obtain $\breve{\varphi}(\mathfrak{g})$, we reverse the arcs between 0 and $\pm \sigma\left(i_{0}\right)$, between 0 and $\pm \sigma\left(i_{0}+2\right)$, between $\sigma\left(i_{0}\right), \sigma\left(i_{0}+1\right)$, and $\sigma\left(i_{0}+2\right)$, and between $-\sigma\left(i_{0}\right),-\sigma\left(i_{0}+1\right)$, and $-\sigma\left(i_{0}+2\right)$. This set of arcs may be written as a disjoint union of cycles:

$$
\begin{aligned}
& \quad\left\{\left(\sigma\left(i_{0}\right), \sigma\left(i_{0}+1\right)\right),\left(\sigma\left(i_{0}+1\right), \sigma\left(i_{0}+2\right)\right),\left(\sigma\left(i_{0}+2\right), 0\right),\left(0, \sigma\left(i_{0}\right)\right)\right\}, \\
& \quad\left\{\left(-\sigma\left(i_{0}\right), 0\right),\left(0,-\sigma\left(i_{0}+2\right)\right),\left(-\sigma\left(i_{0}+2\right),-\sigma\left(i_{0}+1\right)\right),\left(-\sigma\left(i_{0}+1\right),-\sigma\left(i_{0}\right)\right)\right\}, \\
& \text { and } \\
& \left\{\left(\sigma\left(i_{0}\right), \sigma\left(i_{0}+2\right)\right),\left(\sigma\left(i_{0}+2\right),-\sigma\left(i_{0}+2\right)\right),\left(-\sigma\left(i_{0}+2\right),-\sigma\left(i_{0}\right)\right),\left(-\sigma\left(i_{0}\right), \sigma\left(i_{0}\right)\right)\right\} .
\end{aligned}
$$

Now $\breve{\varphi}(\mathfrak{g})^{+}$is transitive, with $\sigma(\breve{\varphi}(\mathfrak{g}))=\sigma \cdot\left(i_{0} i_{0}+2\right)$, and we have $\omega_{0}(\breve{\varphi}(\mathfrak{g}))=$ $\omega_{0}$ and $\omega_{ \pm}(\breve{\varphi}(\mathfrak{g}))=\omega_{ \pm}$. Meanwhile, $i_{0}$ and $i_{0}+2$ are interchangeable in $\tau$, so $\tau(\check{\varphi}(\mathfrak{g}))=\tau$. These observations tell us that $\breve{\varphi}(\check{\varphi}(\mathfrak{g}))=\mathfrak{g}$, and it is easy to see that $\breve{w}(\breve{\varphi}(\mathfrak{g}))=-\breve{w}(\mathfrak{g})$.

We are now done with Phase 3 .

Phase 4

This will be the last phase of our construction of $\tilde{\varphi}$ and $\breve{\varphi}$; we are already finished with $\hat{\varphi}$. 
Construction of $\tilde{\varphi}$. Given $\mathfrak{g} \in \mathfrak{C}_{n} \backslash \mathfrak{C}_{n}^{*}$, with $\mathfrak{g}^{+}$transitive and $\bar{\tau}=\bar{\tau}(\mathfrak{g})$ an order ideal of $\left\langle\begin{array}{c}{[n]} \\ 2\end{array}\right\rangle$, we must have $o(\sigma(i), \mathfrak{g}) \leq o(\sigma(i+1), \mathfrak{g})$ for some $i$. Let $i_{0}$ be the smallest $i$ with this property. Then we have $\#_{0}\left(i_{0}, \tau\right)=\#_{0}\left(i_{0}+1, \tau\right), i_{0} \notin \omega_{ \pm}$, and $i_{0}+1 \in \omega_{ \pm}$. We obtain $\tilde{\varphi}(\mathfrak{g})$ from $\mathfrak{g}$ by reversing the cycle

$$
\left\{\left(\sigma\left(i_{0}\right), \sigma\left(i_{0}+1\right)\right),\left(\sigma\left(i_{0}+1\right),-\sigma\left(i_{0}+1\right)\right),\left(-\sigma\left(i_{0}+1\right),-\sigma\left(i_{0}\right)\right),\left(-\sigma\left(i_{0}\right), \sigma\left(i_{0}\right)\right)\right\} .
$$

Evidently $\tilde{w}(\tilde{\varphi}(\mathfrak{g}))=-\tilde{w}(\mathfrak{g})$. Meanwhile, $\tilde{\varphi}(\mathfrak{g})^{+}$is transitive, with corresponding permutation $\sigma \cdot\left(i_{0} i_{0}+1\right)$, and $\omega_{ \pm}(\tilde{\varphi}(\mathfrak{g}))=\omega_{ \pm}$. Finally, Lemma 5.2(b) tells us that $i_{0}$ and $i_{0}+1$ are interchangeable in $\bar{\tau}$; this means that $\bar{\tau}(\tilde{\varphi}(\mathfrak{g}))=\bar{\tau}$. We conclude that $\tilde{\varphi}(\tilde{\varphi}(\mathfrak{g}))=\mathfrak{g}$. This completes the proof that $\sum_{\mathfrak{g} \in \mathfrak{C}_{n} \backslash \mathfrak{C}_{n}^{*}} \tilde{w}(\mathfrak{g})=0$.

Construction of $\breve{\varphi}$. The only $\mathfrak{g} \in \mathfrak{C}_{n} \backslash \mathfrak{C}_{n}^{* *}$ that remain after Phases $1-3$ are those for which $\mathfrak{g}^{+}$is transitive and $\tau$ is an order ideal of $\left(\begin{array}{c}{[n]} \\ 2\end{array}\right)$, but at least one of the following conditions holds:

(i) $o(\sigma(i), \mathfrak{g})<o(\sigma(i+1), \mathfrak{g})$ for some $i$;

(ii) $\omega_{0} \nsubseteq \omega_{ \pm}$;

(iii) $\omega_{ \pm} \backslash \omega_{0}$ cannot be written as a disjoint union of pairs $\{j, j+1\}$ with the elements of each pair being interchangeable in $\tau$.

For such $\mathfrak{g}$, we shall obtain $\breve{\varphi}(\mathfrak{g})$ by reversing a cycle of one of the following forms:

$$
\begin{aligned}
& \{(\sigma(i), 0),(0,-\sigma(i)),(-\sigma(i), \sigma(i))\}, \\
& \{(\sigma(i),-\sigma(i)),(-\sigma(i), 0),(0, \sigma(i))\},
\end{aligned}
$$

or

$$
\{(\sigma(i), \sigma(i+1)),(\sigma(i+1),-\sigma(i+1)),(-\sigma(i+1),-\sigma(i)),(-\sigma(i), \sigma(i))\} .
$$

It is easy to see that for any $\mathfrak{g}^{\prime}$ obtained from $\mathfrak{g}$ by reversing such a cycle, we have $\breve{w}\left(\mathfrak{g}^{\prime}\right)=-\breve{w}(\mathfrak{g})$. To ensure that $\breve{\varphi}(\mathfrak{g}) \in \mathfrak{C}_{n} \backslash \mathfrak{C}_{n}^{* *}$ and $\breve{\varphi}(\breve{\varphi}(\mathfrak{g}))=\mathfrak{g}$, we need some way of deciding which cycle to reverse. The following discussion explains how this decision is made.

Let $c_{1}=\{i \in[n-1]: o(\sigma(i), \mathfrak{g})<o(\sigma(i+1), \mathfrak{g})\}$ and let $c_{2}=\omega_{0} \backslash \omega_{ \pm}$. These sets correspond to conditions (i) and (ii) above. To describe a set corresponding to (iii) takes a little more effort. We begin by writing $\omega_{ \pm} \backslash \omega_{0}$ as a disjoint union of sets $R_{j}=\left\{i_{j}, i_{j}+1, \ldots, i_{j}+r_{j}-1\right\}$ such that (a), the elements of $R_{j}$ are pairwise interchangeable in $\tau$; and (b), no subset of $\omega_{ \pm} \backslash \omega_{0}$ with elements pairwise interchangeable in $\tau$ contains $R_{j}$ as a proper subset. Observe that $\mathfrak{g} \in \mathfrak{C}_{n}^{* * *}$ only if every $r_{j}$ is even.

Figure 6.4 shows a triple $\left(\tau, \omega_{0}, \omega_{ \pm}\right)$of sets corresponding to some digraphs in $\mathfrak{C}_{6}$. In this and several later figures, the sets $\omega_{0}$ and $\omega_{ \pm}$are described by the squares on the diagonal: the square $(i, i)$ is vertically lined if $i \in \omega_{0}$ and horizontally lined if $i \in \omega_{ \pm}$. Here, we see that $\omega_{ \pm} \backslash \omega_{0}=\{2,3,4,5\}$ and the sets $R_{j}$ are $\{2,3,4\}$ and $\{5\}$. 


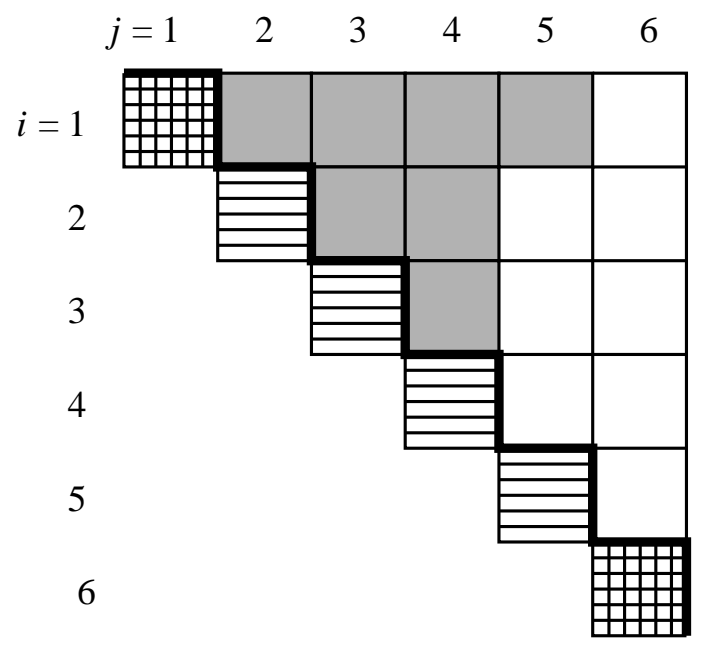

FiguRE 6.4

Having written $\omega_{ \pm} \backslash \omega_{0}$ as a disjoint union of sets $R_{j}$ in the manner described above, let $c_{3}$ be the set containing the largest element of each set $R_{j}$ of odd cardinality. This is the set corresponding to condition (iii). For a digraph corresponding to the sets shown in Figure 6.4, we would have $c_{3}=\{4,5\}$. Observe that the sets $c_{1}, c_{2}$, and $c_{3}$ are pairwise disjoint: $c_{2}=\omega_{0} \backslash \omega_{ \pm}$and $c_{3} \subseteq \omega_{ \pm} \backslash \omega_{0}$, whereas if $i \in c_{1}$, then we must have $i \notin \omega_{0}$ and $i \notin \omega_{ \pm}$.

Now we are ready to define $\breve{\varphi}(\mathfrak{g})$. If $\mathfrak{g}$ survived Phases $1-3$ and the sets $c_{1}$, $c_{2}$, and $c_{3}$ described above are all empty, then $\mathfrak{g}$ is in $\mathfrak{C}_{n}^{* *}$. Otherwise, let $i_{0}=$ $\min \left(c_{1} \cup c_{2} \cup c_{3}\right)$. Exactly one of the following conditions holds:

(1) $i_{0} \in c_{1}$ : in this case, $i_{0} \notin \omega_{0}, i_{0} \notin \omega_{ \pm}, i_{0}+1 \in \omega_{0}, i_{0}+1 \in \omega_{ \pm}$, and $i_{0}$ and $i_{0}+1$ are interchangeable in $\tau$.

(2a) $i_{0} \in c_{2}$, with $i_{0}<n ; i_{0}$ and $i_{0}+1$ interchangeable in $\tau$; and $i_{0}+1 \in \omega_{ \pm} \backslash \omega_{0}$.

(2b) $i_{0} \in c_{2}$, with $i_{0}=n$, or $i_{0}$ and $i_{0}+1$ not interchangeable in $\tau$, or $i_{0}+1 \in \omega_{0}$, or $i_{0}+1 \notin \omega_{ \pm}$.

(3) $i_{0} \in c_{3}$ : in this case, $i_{0}=n$, or $i_{0}$ and $i_{0}+1$ are not interchangeable in $\tau$, or $i_{0}+1 \in \omega_{0}$, or $i_{0}+1 \notin \omega_{ \pm}$.

Suppose (1) holds. Then we obtain $\breve{\varphi}(\mathfrak{g})$ by reversing the cycle

$$
\left\{\left(\sigma\left(i_{0}\right), \sigma\left(i_{0}+1\right)\right),\left(\sigma\left(i_{0}+1\right),-\sigma\left(i_{0}+1\right)\right),\left(-\sigma\left(i_{0}+1\right),-\sigma\left(i_{0}\right)\right),\left(-\sigma\left(i_{0}\right), \sigma\left(i_{0}\right)\right)\right\} .
$$

We find that $\sigma(\breve{\varphi}(\mathfrak{g}))=\sigma \cdot\left(i_{0} i_{0}+1\right)$ and that $\tau(\breve{\varphi}(\mathfrak{g}))=\tau$, since $i_{0}$ and $i_{0}+1$ are interchangeable in $\tau$. Furthermore, $\omega_{0}(\breve{\varphi}(\mathfrak{g}))=\left\{i_{0}\right\} \cup \omega_{0} \backslash\left\{i_{0}+1\right\}$ and $\omega_{ \pm}(\breve{\varphi}(\mathfrak{g}))=$ $\omega_{ \pm}$. These observations imply that when we apply $\breve{\varphi}$ to $\breve{\varphi}(\mathfrak{g})$, we shall find the same $i_{0}$ as before, but condition (2a) will hold.

If $(2 \mathrm{a})$ holds, then we obtain $\breve{\varphi}(\mathfrak{g})$ by reversing the same cycle described in the previous paragraph, and we find that (1) holds for $\breve{\varphi}(\mathfrak{g})$. Combined with the 
previous paragraph, this shows that $\breve{\varphi}(\breve{\varphi}(\mathfrak{g}))=\mathfrak{g}$ for all $\mathfrak{g}$ such that $(1)$ or $(2 \mathrm{a})$ is satisfied.

If (2b) holds, then we obtain $\breve{\varphi}(\mathfrak{g})$ by reversing the cycle

$$
\left\{\left(\sigma\left(i_{0}\right), 0\right),\left(0,-\sigma\left(i_{0}\right)\right),\left(-\sigma\left(i_{0}\right), \sigma\left(i_{0}\right)\right)\right\} .
$$

Evidently this reversal does not change $\sigma$ or $\tau$; it simply removes $i_{0}$ from $\omega_{0}$ and adds it to $\omega_{ \pm}$. So when we apply $\breve{\varphi}$ to $\breve{\varphi}(\mathfrak{g})$, we shall find the same $i_{0}$ as before, and (3) will hold. Finally, if (3) holds, then to obtain $\breve{\varphi}(\mathfrak{g})$, we reverse the cycle

$$
\left\{\left(\sigma\left(i_{0}\right),-\sigma\left(i_{0}\right)\right),\left(-\sigma\left(i_{0}\right), 0\right),\left(0, \sigma\left(i_{0}\right)\right)\right\} ;
$$

condition $(2 \mathrm{~b})$ will hold for the digraph we obtain from this cycle reversal. We conclude that $\breve{\varphi}(\breve{\varphi}(\mathfrak{g}))=\mathfrak{g}$ whenever $\mathfrak{g}$ is such that $(2 \mathrm{~b})$ or $(3)$ is satisfied.

With this, we have completed the proof of Lemma 4.1 .

\section{Correspondence}

We shall prove Lemma 4.2 by showing that the weight of each digraph in $\mathfrak{B}_{n}^{*}$ is a term on the sum side of (BD), and similarly for $\mathfrak{C}_{n}^{*}$ and (BC) and for $\mathfrak{C}_{n}^{* *}$ and (CD).

\section{From Digraphs to Partitions}

For any $\mathfrak{g} \in \mathfrak{B}_{n} \cup \mathfrak{C}_{n}$ with $\mathfrak{g}^{+}$transitive, let $\lambda=\lambda(\mathfrak{g}) \in \mathbf{N}_{0}^{n}$ be such that $\lambda_{i}+n-i=o(\sigma(i), \mathfrak{g})$ for each $i \in[n]$. In other words, $\lambda_{i}=\#(i, \tau)+\chi\left(i \in \omega_{0}\right)$ if $\mathfrak{g} \in \mathfrak{B}_{n}$ and $\lambda_{i}=\#_{0}(i, \bar{\tau})+\chi\left(i \in \omega_{ \pm}\right)=\#(i, \tau)+\chi\left(i \in \omega_{0}\right)+\chi\left(i \in \omega_{ \pm}\right)$if $\mathfrak{g} \in \mathfrak{C}_{n}$. We can express the weight(s) of $\mathfrak{g}$ in terms of $\lambda$. Namely,

$$
\begin{aligned}
& \hat{w}(\mathfrak{g})=(-1)^{\sigma}(-1)^{|\tau|+\left|\omega_{0}\right|} t^{\left|\omega_{0}\right|} x^{\sigma\left(\lambda+\delta_{n}\right)}, \quad \text { for } \mathfrak{g} \in \mathfrak{B}_{n} ; \\
& \tilde{w}(\mathfrak{g})=(-1)^{\sigma}(-1)^{|\bar{\tau}|} t^{\left|\omega_{ \pm}\right|} x^{\sigma\left(\lambda+\delta_{n}\right)}, \quad \text { for } \mathfrak{g} \in \mathfrak{C}_{n} ; \\
& \check{w}(\mathfrak{g})=(-1)^{\sigma}(-1)^{|\tau|+\left|\omega_{0}\right|} t^{\left(\left|\omega_{0}\right|+\left|\omega_{ \pm}\right|\right) / 2} x^{\sigma\left(\lambda+\delta_{n}\right)}, \quad \text { for } \mathfrak{g} \in \mathfrak{C}_{n} .
\end{aligned}
$$

Now if $\mathfrak{g} \in \mathfrak{B}_{n}^{*} \cup \mathfrak{C}_{n}^{*} \cup \mathfrak{C}_{n}^{* *}$, then $o(\sigma(1), \mathfrak{g})>o(\sigma(2), \mathfrak{g})>\cdots>o(\sigma(n), \mathfrak{g})$. This implies that $\lambda(\mathfrak{g})$ is a partition with at most $n$ parts. For $\mathfrak{g} \in \mathfrak{B}_{n}^{*} \cup \mathfrak{C}_{n}^{* * *}$, let $\zeta=(\#(1, \tau), \ldots, \#(n, \tau)) ;$ for $\mathfrak{g} \in \mathfrak{C}_{n}^{*}$, let $\eta=\left(\#_{0}(1, \bar{\tau}), \ldots, \#_{0}(n, \bar{\tau})\right)$. Lemma 5.1 implies that $\zeta \in P_{-1}(n)$ and $\eta \in P_{0}(n)$. We observe that:

If $\mathfrak{g} \in \mathfrak{B}_{n}^{*}$, then $0 \leq \lambda_{i}-\zeta_{i}=\chi\left(i \in \omega_{0}\right) \leq 1$ for all $i \in[n]$.

If $\mathfrak{g} \in \mathfrak{C}_{n}^{*}$, then $0 \leq \lambda_{i}-\eta_{i}=\chi\left(i \in \omega_{ \pm}\right) \leq 1$ for all $i \in[n]$.

If $\mathfrak{g} \in \mathfrak{C}_{n}^{* *}$, then $0 \leq \lambda_{i}-\zeta_{i}=\chi\left(i \in \omega_{0}\right)+\chi\left(i \in \omega_{ \pm}\right) \leq 2$ for all $i \in[n]$. Furthermore, we have $\left\{i: \lambda_{i}-\zeta_{i}=1\right\}=\omega_{ \pm} \backslash \omega_{0}$, which is a disjoint union of pairs $\{j, j+1\}$ such that $j$ and $j+1$ are interchangeable in $\tau$, or equivalently, such that $\zeta_{j}=\zeta_{j+1}$.

The latter observation tells us that $\lambda(\mathfrak{g}) \in P_{-1,1}(n)$ whenever $\mathfrak{g} \in \mathfrak{C}_{n}^{* *}$. We see also that if $\mathfrak{g} \in \mathfrak{B}_{n}^{*}$ (respectively, $\mathfrak{C}_{n}^{*}$ ), then there exists a partition $\xi \in P_{-1}(n)$ (respectively, $P_{0}(n)$ ) such that the skew diagram $\lambda(\mathfrak{g})-\xi$ is a vertical strip. We claim that $\lambda(\mathfrak{g}) \in P_{-1,0}(n)$ if $\mathfrak{g} \in \mathfrak{B}_{n}^{*}$ and $\lambda(\mathfrak{g}) \in P_{0,1}(n)$ if $\mathfrak{g} \in \mathfrak{C}_{n}^{*}$. These are consequences of: 
Lemma 7.1. ([S], Lemma 3.4.2) (a) Let $\lambda=\left(\alpha_{1}, \ldots, \alpha_{p} \mid \beta_{1}, \ldots, \beta_{p}\right)$. Then there exists $\xi \in P_{-1}$ such that $\lambda-\xi$ is a vertical strip if and only if $\beta_{1} \geq \alpha_{1} \geq \beta_{2} \geq \alpha_{2} \geq$ $\cdots \geq \beta_{p} \geq \alpha_{p}$

(b) Let $\lambda=\left(\alpha_{1}, \ldots, \alpha_{p} \mid \beta_{1}, \ldots, \beta_{p}\right)$. Then there exists $\xi \in P_{0}$ such that $\lambda-\xi$ is a vertical strip if and only if $\beta_{1}+1 \geq \alpha_{1} \geq \beta_{2}+1 \geq \alpha_{2} \geq \cdots \geq \beta_{p}+1 \geq \alpha_{p}$.

Statement (b) is due to Okada. The "if" part follows from Lemma 3.7 of [O], and the "only if" part is contained in Lemma 3.8 of the same work. Okada does not state Lemma 7.1(a), but it is implicit in the identity equivalent to (BD) that he states without proof in [O].

Proof of Lemma 7.1(a). "Only if": Suppose $\lambda=\left(\alpha_{1}, \ldots, \alpha_{p} \mid \beta_{1}, \ldots, \beta_{p}\right)$ with either $\alpha_{i}<\beta_{i+1}$ for some $i(1 \leq i \leq p-1)$ or $\beta_{i}<\alpha_{i}$ for some $i(1 \leq i \leq p)$. Let $\xi \in P_{-1}$ be such that $\xi \subseteq \lambda$. If $\bar{\xi}=\left(\gamma_{1}-1, \ldots, \gamma_{q}-1 \mid \gamma_{1}, \ldots, \gamma_{q}\right)$, then $q \leq p$ and we have $\gamma_{j}-1 \leq \alpha_{j}$ and $\gamma_{j} \leq \beta_{j}$ for each $j, 1 \leq j \leq q$. If $\alpha_{i}<\beta_{i+1}$ and $i \leq q$, then we have $\xi_{i}^{\prime}=\gamma_{i}+i<\beta_{i+1}+i+1=\lambda_{i+1}^{\prime}$. Let $k=\beta_{i+1}+i+1$. Then $\lambda_{k} \geq i+1$, but $\xi_{k}<i$. If $\alpha_{i}<\beta_{i+1}$ and $i>q$, then $\alpha_{i}>\alpha_{i+1}$ implies $\alpha_{i} \geq 1$; we have $\lambda_{i} \geq i+1$ but $\xi_{i} \leq q<i$. In either case, $\lambda_{i}-\xi_{i} \geq 2$. And if $\beta_{i}<\alpha_{i}$, we must have $\gamma_{i}<\alpha_{i}$, so that $\lambda_{i}-\xi_{i}=\alpha_{i}-\left(\gamma_{i}-1\right) \geq 2$. We have shown that $\lambda-\xi$ is not a vertical strip.

"If": Given $\lambda=\left(\alpha_{1}, \ldots, \alpha_{p} \mid \beta_{1}, \ldots, \beta_{p}\right)$ with $\beta_{1} \geq \alpha_{1} \geq \beta_{2} \geq \alpha_{2} \geq \cdots \geq \beta_{p} \geq$ $\alpha_{p}$, let $q=p-\chi\left(\beta_{p}=0\right)$. For $1 \leq i \leq q$, let $\gamma_{i}=\alpha_{i}+\chi\left(\beta_{i}>\alpha_{i}\right)$. It is not hard to show that $\gamma_{1}>\gamma_{2}>\cdots>\gamma_{q} \geq 0$ (assuming otherwise, we would conclude that $\beta_{i} \leq \beta_{i+1}$ for some $\left.i\right)$. So it makes sense to define $\xi=\left(\gamma_{1}-1, \ldots, \gamma_{q}-1 \mid \gamma_{1}, \ldots, \gamma_{q}\right)$, and we have $\xi \in P_{-1}$ and $\xi \subseteq \lambda$. For example, if $\lambda=(2,1,0 \mid 4,1,0)$, as in the diagram on the left in Figure 7.1 , then $\xi=(2,0 \mid 3,1)$; if $\lambda=(4,1,0 \mid 4,3,1)$, then $\xi=(3,1,0 \mid 4,2,1)$, as we see in the diagram on the right.
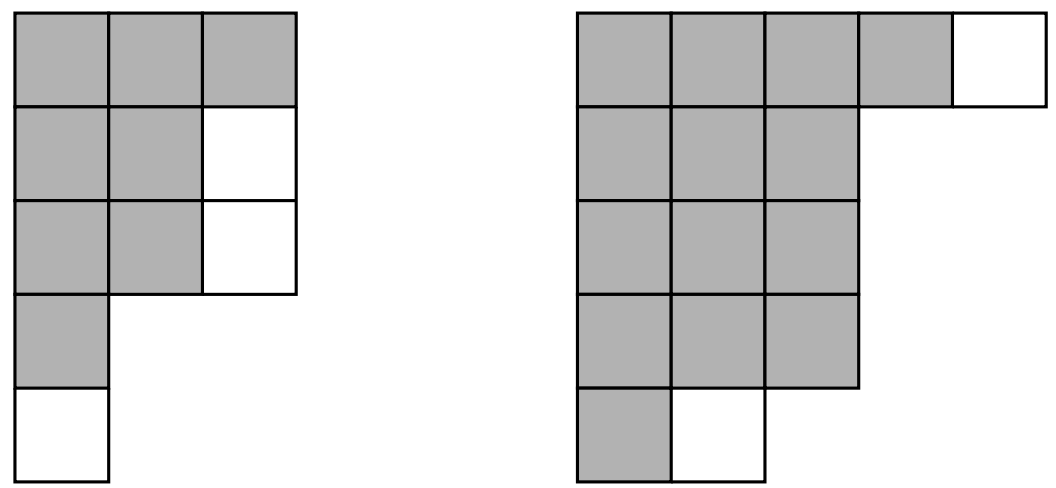

Figure 7.1

Now if $1 \leq i \leq q$, then $\lambda_{i}-\xi_{i}=1-\chi\left(\beta_{i}>\alpha_{i}\right)$. If $q=p-1$, then $\beta_{p}=0$, which implies that $\alpha_{p}=0$ and $\lambda_{p}=p$; meanwhile, we have $\gamma_{p-1} \geq \alpha_{p-1} \geq 1$, so $\xi_{p}=p-1$. We have shown that $0 \leq \lambda_{i}-\xi_{i} \leq 1$ whenever $1 \leq i \leq p$. Suppose $i>p$ and $\lambda_{i}-\xi_{i} \geq 2$. Let $k=\xi_{i}+1$, so that $\xi_{i}<k$ and $p \geq \lambda_{i} \geq k+1$. Then $k+\gamma_{k}=\xi_{k}^{\prime}<i$ and $k+1+\beta_{k+1}=\lambda_{k+1}^{\prime} \geq i$, so we have $\beta_{k+1}+1>\gamma_{k}$. 
If $\gamma_{k}=\alpha_{k}+1$, then $\alpha_{k}<\beta_{k+1}$; otherwise, $\gamma_{k}=\alpha_{k}=\beta_{k}$ and $\beta_{k} \leq \beta_{k+1}$. We conclude from this contradiction that $0 \leq \lambda_{i}-\xi_{i} \leq 1$ for all $i>p$.

The proof of Lemma 7.1(b) is very similar. One way to define $\xi$ in this case is to put $\gamma_{i}=\alpha_{i}-\chi\left(\alpha_{i}>\beta_{i}\right)$ for $1 \leq i \leq p$, then let $\xi=\left(\gamma_{1}, \ldots, \gamma_{p} \mid \gamma_{1}, \ldots, \gamma_{p}\right)$.

Recall that in Section 2, we defined $P_{-1,1}(n)$ in terms of dominos: $\lambda \in P_{-1,1}(n)$ if and only if $\lambda-\kappa$ is a disjoint union of dominos, with no more than one domino per row of $D(\lambda)$, for some $\kappa \in P_{-1}(n)$. If we call any single-element subset of $\mathbf{N}^{2}$ a "monomino," then Lemma 7.1 tells us that $\lambda \in P_{-1,0}(n)$ (respectively, $\lambda \in P_{0,1}(n)$ ) if and only if $D(\lambda) \backslash D(\xi)$ is a disjoint union of monominos, with no more than one such in any one row of $D(\lambda)$, for some $\xi \in P_{-1}(n)$ (respectively, $P_{0}(n)$ ).

By virtue of Lemma 7.1 and formulas (7.1)-(7.3), we have the following:

$$
\sum_{\mathfrak{b} \in \mathfrak{B}_{n}^{*}} \hat{w}(\mathfrak{b})=\left.\sum_{\substack{\lambda \in P_{-1,0}(n) \\ \sigma \in S_{n}}}(-1)^{\sigma} x^{\sigma\left(\lambda+\delta_{n}\right)} \sum_{\tau, \omega_{0}}(-1)^{|\tau|+\left|\omega_{0}\right|}\right|^{\left|\omega_{0}\right|},
$$

where $\lambda_{i}=\#(i, \tau)+\chi\left(i \in \omega_{0}\right)$ for all $i \in[n]$;

$(7.4 \mathrm{bc})$

$$
\sum_{\mathfrak{c} \in \mathfrak{C}_{n}^{*}} \tilde{w}(\mathfrak{c})=\sum_{\substack{\lambda \in P_{0,1}(n) \\ \sigma \in S_{n}}}(-1)^{\sigma} x^{\sigma\left(\lambda+\delta_{n}\right)} \sum_{\bar{\tau}, \omega_{ \pm}}(-1)^{|\bar{\tau}|} t^{\left|\omega_{ \pm}\right|},
$$

where $\lambda_{i}=\#_{0}(i, \bar{\tau})+\chi\left(i \in \omega_{ \pm}\right)$for all $i \in[n]$;

$(7.4 \mathrm{~cd})$

$$
\sum_{\mathfrak{c} \in \mathfrak{C}_{n}^{* *}} \breve{w}(\mathfrak{c})=\sum_{\substack{\lambda \in P_{-1,1}(n) \\ \sigma \in S_{n}}}(-1)^{\sigma} x^{\sigma\left(\lambda+\delta_{n}\right)} \sum_{\tau, \omega_{0}, \omega_{ \pm}}(-1)^{|\tau|+\left|\omega_{0}\right|} t^{\left(\left|\omega_{0}\right|+\left|\omega_{ \pm}\right|\right) / 2},
$$

where $\lambda_{i}=\#(i, \tau)+\chi\left(i \in \omega_{0}\right)+\chi\left(i \in \omega_{ \pm}\right)$for all $i \in[n]$.

In these formulas, $\tau$ and $\bar{\tau}$ are order ideals of $\left(\begin{array}{c}{[n]} \\ 2\end{array}\right)$ and $\left\langle\begin{array}{c}{[n]} \\ 2\end{array}\right\rangle$ respectively, and $\omega_{0}$ and $\omega_{ \pm}$are subsets of $[n]$. We can now complete the proof of Lemma 4.2 by proving:

Lemma 7.2. (bd) For each $\lambda \in P_{-1,0}(n)$, the inner sum on the right side of (7.4bd) is

$$
(-1)^{|\lambda|-|\mu| / 2} t^{|\lambda|-|\mu|}\left(1-t^{2}\right)^{y(\lambda)} \text {. }
$$

(bc) For each $\lambda \in P_{0,1}(n)$, the inner sum on the right side of (7.4bc) is

$$
(-1)^{(|\nu|+p(\nu)) / 2} t^{|\lambda|-|\nu|}(1-t)^{\chi\left(\exists i, \lambda_{i}=i\right)}\left(1-t^{2}\right)^{z(\lambda)} .
$$

(cd) For each $\lambda \in P_{-1,1}(n)$, the inner sum on the right side of (7.4cd) is

$$
(-1)^{|\kappa| / 2+q(\lambda)} t^{(|\lambda|-|\kappa|) / 2}(1-t)^{\chi\left(\exists i, \lambda_{i}=i\right)}\left(1-t^{2}\right)^{z(\lambda)} .
$$


Proofs of Lemmas 2.1 AND 2.3

We require Lemma 2.1 in our proofs of (bd) and (bc) and Lemma 2.3 in our proof of $(\mathrm{cd})$. Since we have not yet proved these lemmas, we do so now. It should be mentioned that the proof in [S] of Lemma 3.1.3, which is the same as Lemma 2.3 here, is invalid.

Proof of Lemma 2.1. (a) Let $\lambda \in P_{-1,0}(n)$ with Frobenius representation $\left(\alpha_{1}, \ldots\right.$, $\left.\alpha_{p} \mid \beta_{1}, \ldots, \beta_{p}\right)$. We claim that $\mu(\lambda) \subseteq \lambda$. Suppose $i, j$ are such that $i<j$ and $(i, j-1) \notin D(\lambda)$. We have $j-1>\lambda_{i}$, so $\lambda_{i}+\lambda_{j}<\lambda_{j}+j-1$. If $i \geq p$, then we have $j>p$ and $\lambda_{j} \leq p \leq i$. If $i<p$, then $j>\lambda_{i}+1=\alpha_{i}+i+1 \geq \beta_{i+1}+i+1=\lambda_{i+1}^{\prime}$, which means that $\lambda_{j}<i+1$. In either case, $\lambda_{i}+\lambda_{j}<i+j-1$, so $(i, j-1) \notin D(\mu)$. If $i<j$ and $(j, i) \notin D(\lambda)$, then $\lambda_{i}+\lambda_{j}<\lambda_{i}+i$, and we shall show that $\lambda_{i} \leq j-1$. If $i>p$, then $\lambda_{i}<i \leq j-1$; if $i \leq p$, then $\lambda_{i}=\alpha_{i}+i \leq \beta_{i}+i=\lambda_{i}^{\prime}$, and $\lambda_{i}^{\prime} \leq j-1$ since $(j, i) \notin D(\lambda)$. Again we have $\lambda_{i}+\lambda_{j}<i+j-1$, and $(j, i) \notin D(\mu)$. This proves our claim. It remains to show that if $\xi \subseteq \lambda$ and $\xi \in P_{-1}$, then $\xi \subseteq \mu$. This is fairly easy. If $\xi \in P_{-1}$ and $\xi \not \subset \mu$, then $(i, j-1),(j, i) \in D(\xi) \backslash D(\mu)$ for some $i, j$ with $i<j$. For such $i, j$ we have $\lambda_{i}+\lambda_{j}<i+j-1$, but if both $(i, j-1)$ and $(j, i)$ are in $D(\lambda)$, then $\lambda_{i} \geq j-1$ and $\lambda_{j} \geq i$, meaning that $\lambda_{i}+\lambda_{j} \geq i+j-1$. So $(i, j-1)$ and $(j, i)$ are not both in $D(\lambda)$, and we conclude $\xi \nsubseteq \lambda$.

(b) The proof is much like that of (a). Let $\lambda=\left(\alpha_{1}, \ldots, \alpha_{p} \mid \beta_{1}, \ldots, \beta_{p}\right) \in$ $P_{0,1}(n)$. If $(i, j) \notin D(\lambda)$, then $j>\lambda_{i}$. We shall show that $i \geq \lambda_{j}$, from which it will follow that $(i, j) \notin D(\nu)$. If $i<p$, then $j>\lambda_{i}=\alpha_{i}+i \geq \beta_{i+1}+i+1=\lambda_{i+1}^{\prime}$, from which we conclude $\lambda_{j}<i+1$. If $i \geq p$, then if $j>p$, then $\lambda_{j} \leq p \leq i$. Otherwise, $j \leq p$; write $\lambda_{j}^{\prime}+1=\beta_{j}+j+1 \geq \alpha_{j}+j=\lambda_{j}$, and observe that since $(i, j) \notin D(\lambda)$, we have $i \geq \lambda_{j}^{\prime}+1$. We have shown that $\nu \subseteq \lambda$, and the proof that $\xi \subseteq \nu$ for any other $\xi \in P_{0}$ such that $\xi \subseteq \lambda$ is easy.

Proof of Lemma 2.3. Let $\lambda \in P_{-1,1}(n)$. To prove that there is a partition $\kappa \in K(\lambda)$ such that $\eta \subseteq \kappa$ for all $\eta \in K(\lambda)$, we shall show that if $\kappa, \eta \in K(\lambda)$ with $\eta \nsubseteq \notin \kappa$, then there exists $\bar{\kappa} \in K(\lambda)$ such that $D(\kappa)$ is strictly smaller than $D(\bar{\kappa})$. We shall describe $\bar{\kappa}$ in two ways: by giving its Frobenius representation and by identifying the dominos that are removed from $\lambda-\kappa$ to give $\lambda-\bar{\kappa}$.

Write $\kappa=\left(\alpha_{1}-1, \ldots, \alpha_{p}-1 \mid \alpha_{1}, \ldots, \alpha_{p}\right)$ and $\eta=\left(\beta_{1}-1, \ldots, \beta_{q}-1 \mid \beta_{1}, \ldots\right.$, $\left.\beta_{q}\right)$. There are two ways in which we can have $\eta \nsubseteq \kappa:(1) \alpha_{i} \geq \beta_{i}$ for all $i \in[p]$, but $p<q$; and (2) $\alpha_{i}<\beta_{i}$ for some $i \leq \min \{p, q\}$. We consider these two cases separately.

In case (1), we must have $\alpha_{p} \geq 2$, meaning $\kappa_{p}>\kappa_{p+1}=\kappa_{p+2}=p$. Since $0 \leq \lambda_{i}-\kappa_{i} \leq 2$ for all $i \in[n]$, we have $p+1 \leq \eta_{p+1} \leq \lambda_{p+1} \leq p+2$ and $p+1 \leq \eta_{p+2} \leq \lambda_{p+2} \leq p+2$. We consider three subcases:

(i) $\lambda_{p+1}=p+1$ : We have $\lambda_{p+2}=p+1$, since $\kappa$ would not be in $K(\lambda)$ otherwise. Let $\bar{\kappa}=\left(\alpha_{1}-1, \ldots, \alpha_{p}-1,0 \mid \alpha_{1}, \ldots, \alpha_{p}, 1\right)$.

Dominos: Remove $\{(p+1, p+1),(p+2, p+1)\}$. 
(ii) $\eta_{p+1}=p+1, \lambda_{p+1}=p+2$ : We have $\beta_{p+1}=1$, so $\eta_{p+2}=p+1$. If $\lambda_{p+2}=p+1$, then we must have $\eta_{p}=p+1$ (otherwise $\eta$ could not be in $K(\lambda)$ ) and $\lambda_{p}=p+2$. Meanwhile, $\lambda_{p+1}-\kappa_{p+1}=2$ and $\lambda_{p+2}-\kappa_{p+2}=1$, so we must have $\lambda_{p+3}=p+1$ and $\kappa_{p+3}=\kappa_{p+2}=p$. This tells us that $\alpha_{p} \geq 3$ (in fact, $\alpha_{p}=3$, since otherwise $\left.\kappa_{p}>p+2=\lambda_{p}\right)$. Let $\bar{\kappa}=\left(\alpha_{1}-1, \ldots, \alpha_{p}-1,1 \mid \alpha_{1}, \ldots, \alpha_{p}, 2\right)$. If $\lambda_{p+2}=p+2$, then let $\bar{\kappa}=\left(\alpha_{1}-1, \ldots, \alpha_{p}-1,0 \mid \alpha_{1}, \ldots, \alpha_{p}, 1\right)$.

Dominos: If $\lambda_{p+2}=p+1$, remove $\{(p+1, p+1),(p+1, p+2)\}$ and $\{(p+$ $2, p+1),(p+3, p+1)\}$. If $\lambda_{p+2}=p+2$, replace $\{(p+1, p+1),(p+1, p+2)\}$ and $\{(p+2, p+1),(p+2, p+2)\}$ with $\{(p+1, p+2),(p+2, p+2)\}$.

(iii) $\eta_{p+1}=p+2$ : We have $\eta_{p+2}=\eta_{p+3}=p+1$. Since $\lambda_{p+1}=\eta_{p+1}$ in this case, we must have $p+1 \leq \lambda_{p+2}=\lambda_{p+3} \leq p+2$ in order that $\eta$ be in $K(\lambda)$. We must also have $\alpha_{p} \geq 3$, since $\beta_{p+1}=2$. Let $\bar{\kappa}=\left(\alpha_{1}-1, \ldots, \alpha_{p}-1,1 \mid \alpha_{1}, \ldots, \alpha_{p}, 2\right)$.

Dominos: Remove $\{(p+1, p+1),(p+1, p+2)\}$; if $\lambda_{p+2}=p+1$, remove $\{(p+2, p+1),(p+3, p+1)\}$, otherwise, replace $\{(p+2, p+1),(p+2, p+2)\}$ and $\{(p+3, p+1),(p+3, p+2)\}$ with $\{(p+2, p+2),(p+3, p+2)\}$.

We are now done with (1).

In case (2), choose the smallest $i$ for which $\alpha_{i}<\beta_{i}$. We have $\alpha_{j} \geq \beta_{j}$ for all $j<i$, meaning that $\kappa_{j} \geq \eta_{j}$ whenever $j<i$ or $j>i+\beta_{i}$ and that $\kappa_{i-1} \geq \eta_{i-1} \geq \eta_{i}>\kappa_{i}$ if $i>1$.

Given a subset $X$ of $\mathbf{N}^{2}$ and a domino $D$, we shall say that $D$ crosses the border of $X$ if one of the two elements of $D$ is in $X$ and the other is not. Observe that whenever $\zeta \in K(\lambda)$, the cardinality of $X \cap(\lambda-\zeta)$ is congruent modulo 2 to the number of dominos in $\lambda-\zeta$ that cross the border of $X$. If $X$ is a subset of $\mathbf{N}^{2}$ such that $X \cap D(\zeta)$ has even cardinality whenever $\zeta \in P_{-1}$, then we have (7.5)

$$
\begin{aligned}
\#(\text { dominos in } \lambda-\zeta \text { that cross the border of } X) & \equiv|X \cap(\lambda-\zeta)| \\
& \equiv|X \cap D(\lambda)|-|X \cap D(\zeta)| \\
& \equiv|X \cap D(\lambda)|
\end{aligned}
$$

for each $\zeta \in K(\lambda)$, where $\equiv$ stands for congruence modulo 2. We observe that if $X$ can be written as a (possibly infinite) union of sets of the form $\{(k, l-1),(l, k)$ : $k<l\}$, then $|X \cap D(\zeta)|$ is even for all $\zeta \in P_{-1}$.

We now begin the description of $\bar{\kappa}$ in case (2). Let $r=i+\alpha_{i}$ in what follows. There are two subcases to consider:

(i) $\alpha_{i}=\beta_{i}-2$ : We have $\lambda_{i}=\eta_{i}=\kappa_{i}+2, \kappa_{r}>\kappa_{r+1}=\kappa_{r+2}=i-1$, $\eta_{r+1} \geq \eta_{r+2} \geq i$, and $i+1 \geq \lambda_{r+1} \geq \lambda_{r+2} \geq i$.

Let $X=\left\{(k, l) \in \mathbf{N}^{2}: k, l>i\right\}$; then $|X \cap D(\zeta)|$ is even for all $\zeta \in P_{-1}$. Since $\lambda_{i}-\kappa_{i} \neq 1$, there is no "vertical" domino in $\lambda-\kappa$ that crosses the border of $X$. Similarly, no vertical domino in $\lambda-\eta$ crosses the border of $X$. Any "horizontal" domino in $\lambda-\eta$ that crosses the border of $X$ is of the form $\{(k, i),(k, i+1)\}$ for some $k>r+2$ such that $\eta_{k}=i-1$ and $\lambda_{k}=i+1$. Since $r+2=i+\beta_{i}$, we see that $i-1=\eta_{k} \leq \kappa_{k} \leq \kappa_{r+2}=i-1$. This means that the domino $\{(k, i),(k, i+1)\}$ is also in $\lambda-\kappa$. Now (7.5) implies that the number of dominos 
in $\lambda-\kappa$ that cross the border of $X$ is congruent modulo 2 to the number of such dominos in $\lambda-\eta$. So we must have an even number of horizontal dominos in $\lambda-\kappa$ that cross the border of $X$ and are not in $\lambda-\eta$. The only possible dominos with these properties are $\{(r+1, i),(r+1, i+1)\}$ and $\{(r+2, i),(r+2, i+1)\}$; either $\lambda-\kappa$ contains both of these (in which case $\lambda_{r+1}=i+1$ ), or it contains neither (in which case $\lambda_{r+1}=i$ ). In each case, we obtain $\bar{\kappa}$ by replacing $\alpha_{i}$ with $\beta_{i}=\alpha_{i}+2$. In terms of dominos, we remove $\left\{\left(i, \kappa_{i}+1\right),\left(i, \kappa_{i}+2\right)\right\}$ from $\lambda-\kappa$; if $\lambda_{r+1}=i+1$, then we replace $\{(r+1, i),(r+1, i+1)\}$ and $\{(r+2, i),(r+2, i+1)\}$ with $\{(r+1, i+1),(r+2, i+1)\}$, otherwise, we remove $\{(r+1, i),(r+2, i)\}$.

(ii) $\alpha_{i}=\beta_{i}-1$ : We have $\kappa_{r}>\kappa_{r+1}=i-1$ and $\eta_{r+1} \geq i>\eta_{r+2}$. Either $\lambda_{r+1}=i+1$ or $\lambda_{r+1}=i$. We consider these subsubcases separately.

If $\lambda_{r+1}=i+1$, let $X=\left\{(k, l) \in \mathbf{N}^{2}: k, l>i\right\}$. The horizontal domino $\{(r+1, i),(r+1, i+1)\}$ crosses the border of $X$; it is in $\lambda-\kappa$ but not in $\lambda-\eta$. Any horizontal domino in $\lambda-\eta$ that crosses the border of $X$ must be of the form $\{(k, i),(k, i+1)\}$ for some $k>r+1$ such that $\eta_{k}=i-1$ and $\lambda_{k}=i+1$. Such a domino must also be in $\lambda-\kappa$. So the number of horizontal dominos in $\lambda-\kappa$ that cross the border of $X$ is one greater than the number of such dominos in $\lambda-\eta$. Now (7.5) implies that the number of vertical dominos in $\lambda-\kappa$ that cross the border of $X$ must differ from the number of such dominos in $\lambda-\eta$ by an odd integer. Since at most one vertical domino in either $\lambda-\kappa$ or $\lambda-\eta$ can cross the border of $X$, we either have the domino $\left\{\left(i, \kappa_{i}+1\right),\left(i+1, \kappa_{i}+1\right)\right\}$ in $\lambda-\kappa$ (in which case $\lambda_{i}=\eta_{i}=\kappa_{i}+1$ ) or the domino $\left\{\left(i, \eta_{i}+1\right),\left(i+1, \eta_{i}+1\right)\right\}$ in $\lambda-\eta$ (in which case $\lambda_{i}=\eta_{i}+1=\kappa_{i}+2$ ). In each case, we obtain $\bar{\kappa}$ from $\kappa$ by replacing $\alpha_{i}$ with $\alpha_{i}+1$ and $\alpha_{i+1}$ with $\alpha_{i+1}+1$. (If $i=p$, then instead of the latter replacement, we add to the rank of $\kappa$ by appending $(0 \mid 1)$ to its Frobenius representation.) To obtain $\lambda-\bar{\kappa}$ from $\lambda-\kappa$, we remove $\{(r+1, i),(r+1, i+1)\}$, and we either remove $\left\{\left(i, \kappa_{i}+1\right),\left(i+1, \kappa_{i}+1\right)\right\}$ or replace $\left\{\left(i, \kappa_{i}+1\right),\left(i, \kappa_{i}+2\right)\right\}$ and $\left\{\left(i+1, \kappa_{i}+1\right),\left(i+1, \kappa_{i}+2\right)\right\}$ with $\left\{\left(i, \kappa_{i}+2\right),\left(i+1, \kappa_{i}+2\right)\right\}$ according as $\lambda_{i}-\kappa_{i}$ is 1 or 2 .

If $\lambda_{r+1}=i$, then let $X=[r+1] \times[r]$. Observe that $r=\kappa_{i}+1$. Since $\kappa_{r}>\kappa_{r+1}=\lambda_{r+1}-1, \lambda-\kappa$ must contain the vertical domino $\{(r+1, i),(r+2, i)\}$, which crosses the border of $X$. There is no vertical domino in $\lambda-\eta$ that crosses the border of $X$. Suppose $\{(k, r),(k, r+1)\}$ is a horizontal domino in $\lambda-\eta$ that crosses the border of $X$. Then $k>i$, since $\eta_{i}=\kappa_{i}+1=r$. We have $\kappa_{k} \leq \kappa_{i}=r-1$; since $\lambda_{k}=r+1$, we conclude $\kappa_{k}=r-1$. This means that $\{(k, r),(k, r+1)\}$ is also a domino in $\lambda-\kappa$. Now (7.5) tells us we must have an odd number of horizontal dominos in $\lambda-\kappa$ that cross the border of $X$ and are not in $\lambda-\eta$. The only possible domino with these properties is $\{(i, r),(i, r+1)\}$, since $\kappa_{k} \geq r$ whenever $k<i$. We obtain $\lambda-\bar{\kappa}$ from $\lambda-\kappa$ by removing $\{(r+1, i),(r+2, i)\}$ and $\{(i, r),(i, r+1)\}$; the Frobenius representation of $\bar{\kappa}$ is obtained from that of $\kappa$ by replacing $\alpha_{i}$ with $\alpha_{i}+2$.

This completes subcase (ii) of case (2); we have finished the proof of Lemma 2.3 . 


\section{Proof of Lemma $7.2(\mathrm{bd})$}

For each $\lambda \in P_{-1,0}(n)$, we must identify which partitions $\xi \in P_{-1}(n)$ are such that $\lambda-\xi$ is a vertical strip. For example, Figure 7.2 shows that there are four such partitions $\xi$ corresponding to $\lambda=(4,2,2,2,1) \in P_{-1,0}(5)$.
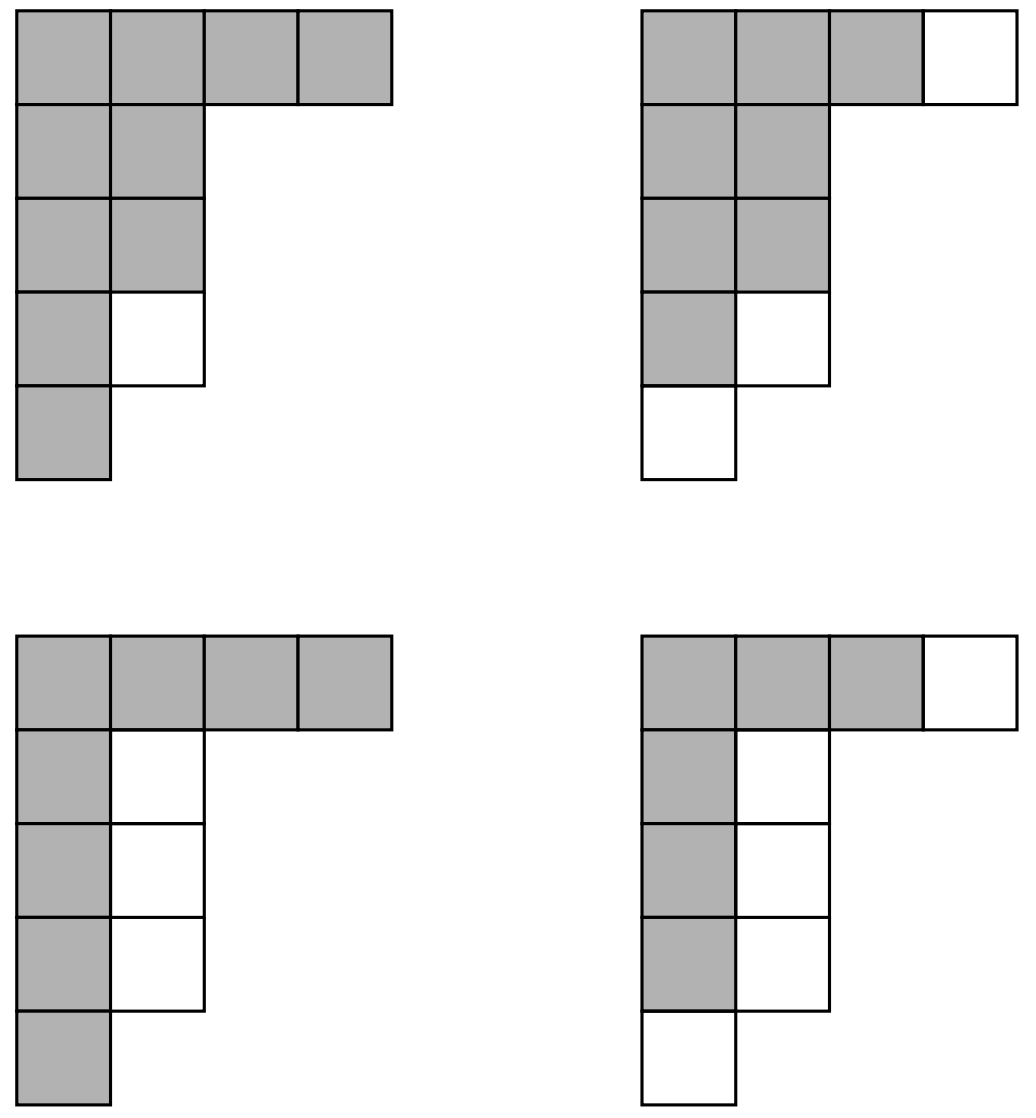

FIGURE 7.2

Let $\lambda \in P_{-1,0}(n)$. Suppose $\xi, \xi^{*} \in P_{-1}(n)$ are such that $\lambda-\xi$ and $\lambda-\xi^{*}$ are vertical strips and $\xi^{*} \subseteq \xi \subseteq \lambda$. Let $\tau$ and $\tau^{*}$ be the order ideals of $\left(\begin{array}{c}{[n]} \\ 2\end{array}\right)$ that correspond to $\xi$ and $\xi^{*}$ in the sense of Lemma 5.1(a), and let $\omega_{0}=\{i \in[n]$ : $\left.\lambda_{i}-\xi_{i}=1\right\}$ and $\omega_{0}^{*}=\left\{i \in[n]: \lambda_{i}-\xi_{i}^{*}=1\right\}$. We have $\tau^{*} \subseteq \tau$ and $\omega_{0}^{*} \supseteq \omega_{0}$; if the elements of $\omega_{0}^{*} \backslash \omega_{0}$ are $i_{1}<i_{2}<\cdots<i_{2 r}$, then $\tau \backslash \tau^{*}=\left\{\left(i_{1}, i_{2 r}\right),\left(i_{2}, i_{2 r-1}\right)\right.$, $\left.\ldots,\left(i_{r}, i_{r+1}\right)\right\}$. This means that if $\mathfrak{g}, \mathfrak{g}^{*} \in \mathfrak{B}_{n}^{*}$ are such that $\mathfrak{g}^{+}=\left(\mathfrak{g}^{*}\right)^{+}, \tau=\tau(\mathfrak{g})$, $\tau^{*}=\tau\left(\mathfrak{g}^{*}\right), \omega_{0}=\omega_{0}(\mathfrak{g})$, and $\omega_{0}^{*}=\omega_{0}\left(\mathfrak{g}^{*}\right)$, then $\hat{w}\left(\mathfrak{g}^{*}\right)=\left(-t^{2}\right)^{\left|\tau \backslash \tau^{*}\right|} \hat{w}(\mathfrak{g})$. Also, if $(i, j) \in \tau \backslash \tau^{*}$, then there is no other ordered pair in $\tau \backslash \tau^{*}$ having $i$ or $j$ as one of its components. So we see that if $(i, j) \in \tau \backslash \tau^{*}$, then $(i, j) \in I(\tau)$ : otherwise, $\tau \backslash \tau^{*}$ would contain $(i, j+1)$ or $(i+1, j)$, or $\tau^{*}$ would not be an order ideal of $\left(\begin{array}{c}{[n]} \\ 2\end{array}\right)$. These observations imply that $\tau \backslash \tau^{*}$ is a subset of $Y\left(\tau, \omega_{0}\right)=\left\{(i, j) \in I(\tau): i, j \notin \omega_{0}\right\}$. 
Fix $\sigma \in S_{n}$ and $\lambda \in P_{-1,0}(n)$, and let $\xi$ be the largest partition in $P_{-1}(n)$ such that $\lambda-\xi$ is a vertical strip. Let $\tau$ be the order ideal of $\left(\begin{array}{c}{[n]} \\ 2\end{array}\right)$ corresponding to $\xi$ and let $\omega_{0}=\left\{i \in[n]: \lambda_{i}-\xi_{i}=1\right\}$. Observe that $|\lambda|=2|\tau|+\left|\omega_{0}\right|$ and $|\xi|=2|\tau|$. We conclude from the discussion in the previous paragraph that $\sum \hat{w}(\mathfrak{g})$, where $\mathfrak{g}$ ranges over all digraphs in $\mathfrak{B}_{n}^{*}$ such that $o\left(\sigma(i), \mathfrak{g}^{+}\right)=n-i$ and $o(\sigma(i), \mathfrak{g})=\lambda_{i}+n-i$, is

$$
\begin{aligned}
& (-1)^{\sigma} x^{\sigma\left(\lambda+\delta_{n}\right)}(-1)^{|\tau|+\left|\omega_{0}\right|} t^{\left|\omega_{0}\right|} \sum_{A \subseteq Y\left(\tau, \omega_{0}\right)}\left(-t^{2}\right)^{|A|} \\
& =(-1)^{\sigma} x^{\sigma\left(\lambda+\delta_{n}\right)}(-1)^{|\lambda|-|\xi| / 2} t^{|\lambda|-|\xi|}\left(1-t^{2}\right)^{\left|Y\left(\tau, \omega_{0}\right)\right|} .
\end{aligned}
$$

For example, if $\lambda$ is as in Figure 7.2 , then $\xi=(4,2,2,1,1)$ and $Y\left(\tau, \omega_{0}\right)=$ $\{(1,5),(2,3)\}$. We shall now prove that $\xi=\mu(\lambda)$ and that $\left|Y\left(\tau, \omega_{0}\right)\right|=y(\lambda)$; this will complete the proof of Lemma $7.2(\mathrm{bd})$.

Lemma 2.1(a) tells us that $\mu(\lambda)$ is the largest partition in $P_{-1}(n)$ whose Ferrers diagram is contained in $D(\lambda)$. To conclude that $\xi=\mu(\lambda)$, we need only show that $\lambda-\mu$ is a vertical strip. Write $\lambda=\left(\alpha_{1}, \ldots, \alpha_{p} \mid \beta_{1}, \ldots, \beta_{p}\right)$ and suppose that $\lambda_{i}-\mu_{i}>1$ for some $i \in[n]$. Then $\left(i, \lambda_{i}-1\right) \notin D(\mu)$. If $i<\lambda_{i}$, then we have $\lambda_{i}+\lambda_{\lambda_{i}}<i+\lambda_{i}-1$; otherwise, $i>\lambda_{i}-1$ and we have $\lambda_{\lambda_{i}-1}+\lambda_{i}<i+\lambda_{i}-2$. In the first case, $\lambda_{\lambda_{i}}<i-1$, which means $\lambda_{i-1}^{\prime}<\lambda_{i}$. Since $i<\lambda_{i}$, we must have $i \leq p$, so

$$
\lambda_{i-1}^{\prime}=i-1+\beta_{i-1}<i+\alpha_{i}=\lambda_{i} .
$$

This implies $\alpha_{i-1}>\beta_{i-1}$, meaning $\lambda \notin P_{-1,0}(n)$. In the second case, $\lambda_{\lambda_{i}-1}<i-2$, meaning $\lambda_{i-2}^{\prime}<\lambda_{i}-1$. We have $\lambda_{i} \leq i$ and $\lambda_{i-2}^{\prime} \leq \lambda_{i}-2 \leq i-2$, so $\lambda_{i}=\max \{j \in$ $\left.[p]: j+\beta_{j} \geq i\right\}$ and $\lambda_{i-2}^{\prime}=\max \left\{j \in[p]: j+\alpha_{j} \geq i-2\right\}$. Let $k=\lambda_{i}-1$. Then $k<p$, since $\lambda_{i} \leq i$ implies $\lambda_{i} \leq p$; we have

$$
k+\alpha_{k}<i-2<i \leq k+1+\beta_{k+1}
$$

so $\alpha_{k}<\beta_{k+1}$ and $\lambda \notin P_{-1,0}(n)$. We have shown that if $\lambda \in P_{-1,0}(n)$, then $\lambda_{i}-\mu_{i} \leq 1$ for all $i \in[n]$, as required.

Let $\tau$ be the order ideal of $\left(\begin{array}{c}{[n]} \\ 2\end{array}\right)$ corresponding to $\mu(\lambda)$ and let $\omega_{0}=\left\{i: \lambda_{i}-\mu_{i}=\right.$ 1\}. We shall show that $\left|Y\left(\tau, \omega_{0}\right)\right|=y(\lambda)$. For any $(i, j) \in\left(\begin{array}{c}{[n]} \\ 2\end{array}\right)$, we have $(i, j) \in \tau$ if and only if $(i, j-1) \in D(\mu)$ if and only if $\lambda_{i}+\lambda_{j} \geq i+j-1$. If $(i, j) \in I(\tau)$, then $\#(i, \tau)=j-1$ and $\#(j, \tau)=i$, so $\mu_{i}+\mu_{j}=i+j-1$; if in addition $i, j \notin \omega_{0}$, then $\lambda_{i}+\lambda_{j}=i+j-1$. Conversely, suppose $i<j$ and $\lambda_{i}+\lambda_{j}=i+j-1$. Then $(i, j-1) \in D(\mu)$ and $(i, j) \in \tau$, implying that $\#(i, \tau) \geq j-1$ and $\#(j, \tau) \geq i$, so we must have $i, j \notin \omega_{0}$. Furthermore, $\lambda_{i+1}+\lambda_{j}$ and $\lambda_{i}+\lambda_{j+1}$ are both at most $i+j-1$, so $(i, j+1)$ and $(i+1, j)$ are not in $\tau$; we conclude that $(i, j) \in I(\tau)$. We have shown

$$
Y\left(\tau, \omega_{0}\right)=\left\{(i, j): i<j, \lambda_{i}+\lambda_{j}=i+j-1\right\}
$$

and $y(\lambda)$ is defined to be the cardinality of this set.

With this, we have completed the proof of Lemma 7.2(bd) and of (BD). 


\section{Proof of Lemma $7.2(\mathrm{bc})$}

This proof is much like the preceding one. We must identify, for each $\lambda \in P_{0,1}(n)$, those $\xi \in P_{0}(n)$ for which $\lambda-\xi$ is a vertical strip. Given such a $\lambda$, suppose $\xi, \xi^{*} \in P_{0}(n)$ are such that $\lambda-\xi$ and $\lambda-\xi^{*}$ are vertical strips and $\xi^{*} \subseteq \xi \subseteq \lambda$. Let $\bar{\tau}$ and $\bar{\tau}^{*}$ be the order ideals of $\left\langle\begin{array}{c}{[n]} \\ 2\end{array}\right\rangle$ corresponding to $\xi$ and $\xi^{*}$ in the sense of Lemma 5.1(b). Let $\omega_{ \pm}=\left\{i \in[n]: \lambda_{i}-\xi_{i}=1\right\}$ and $\omega_{ \pm}^{*}=\left\{i \in[n]: \lambda_{i}-\xi_{i}^{*}=1\right\}$. We find that $\bar{\tau} \backslash \bar{\tau}^{*}$ is a subset of $\bar{Z}\left(\bar{\tau}, \omega_{ \pm}\right)=\left\{(i, j) \in I(\bar{\tau}): i, j \notin \omega_{ \pm}\right\}$.

Observe that it is possible to have $(i, i) \in I(\bar{\tau})$ for some $i$. If $\tau=\bar{\tau} \cap\left(\begin{array}{c}{[n]} \\ 2\end{array}\right)$ and $\tau^{*}=\bar{\tau}^{*} \cap\left(\begin{array}{c}{[n]} \\ 2\end{array}\right)$, then we have

$$
\tilde{w}\left(\mathfrak{g}^{*}\right)=\left(-t^{2}\right)^{\left|\tau \backslash \tau^{*}\right|}(-t)^{\chi\left((i, i) \in \bar{\tau} \backslash \bar{\tau}^{*}\right)} \tilde{w}(\mathfrak{g}),
$$

whenever $\mathfrak{g}, \mathfrak{g}^{*} \in \mathfrak{C}_{n}^{*}$ are such that $\mathfrak{g}^{+}=\left(\mathfrak{g}^{*}\right)^{+}, \bar{\tau}=\bar{\tau}(\mathfrak{g}), \bar{\tau}^{*}=\bar{\tau}\left(\mathfrak{g}^{*}\right), \omega_{ \pm}=\omega_{ \pm}(\mathfrak{g})$, and $\omega_{ \pm}^{*}=\omega_{ \pm}\left(\mathfrak{g}^{*}\right)$. Now if $(i, i) \in I(\bar{\tau})$, then $\#_{0}(i, \bar{\tau})=i$; if in addition $i \notin \omega_{ \pm}$, then $\lambda_{i}=i$. Conversely, if $\lambda_{i}=i$ and $\xi \in P_{0}(n)$ is such that $\lambda-\xi$ is a vertical strip, and if $\bar{\tau}$ is the order ideal of $\left\langle\begin{array}{c}{[n]} \\ 2\end{array}\right\rangle$ corresponding to $\xi$, then either $\xi_{i}=i$ and $(i, i) \in I(\bar{\tau})$, or $\xi_{i}=i-1$ and $(i, i) \in O(\bar{\tau})$. This means that the case $(i, i) \in I(\bar{\tau})$, $i \notin \omega_{ \pm}$can occur if and only if $\lambda_{i}=i$ for some $i \in[n]$, and otherwise the exponent of $t$ on the right side of $(\dagger)$ must be even.

Let $Z\left(\bar{\tau}, \omega_{ \pm}\right)=\bar{Z}\left(\bar{\tau}, \omega_{ \pm}\right) \cap\left(\begin{array}{c}{[n]} \\ 2\end{array}\right)$. Fix $\sigma \in S_{n}$ and $\lambda \in P_{0,1}(n)$; let $\xi$ be the largest partition in $P_{0}(n)$ such that $\lambda-\xi$ is a vertical strip. Let $\bar{\tau}$ denote the order ideal of $\left\langle\begin{array}{c}{\left[\begin{array}{c}n \\ 2\end{array}\right.} \\ \rangle\end{array}\right\rangle$ corresponding to $\xi$ and $\omega_{ \pm}$the set $\left\{i: \lambda_{i}-\xi_{i}=1\right\}$. The sum of $\tilde{w}(\mathfrak{g})$, as $\mathfrak{g}$ ranges over all digraphs in $\mathfrak{C}_{n}^{*}$ such that $o\left(\sigma(i), \mathfrak{g}^{+}\right)=n-i$ and $o(\sigma(i), \mathfrak{g})=\lambda_{i}+n-i$, is

$$
(-1)^{\sigma} x^{\sigma\left(\lambda+\delta_{n}\right)}(-1)^{\mid \bar{\tau} t^{\left|\omega_{ \pm}\right|}}(1-t)^{\chi\left(\exists i, \lambda_{i}=i\right)}\left(1-t^{2}\right)^{\left|Z\left(\bar{\tau}, \omega_{ \pm}\right)\right|} .
$$

Evidently $|\bar{\tau}|=(|\xi|+p(\xi)) / 2$ and $\left|\omega_{ \pm}\right|=|\lambda|-|\xi|$, so we need only show that $\xi=\nu(\lambda)$ and $\left|Z\left(\bar{\tau}, \omega_{ \pm}\right)\right|=z(\lambda)$ to finish the proof of Lemma 7.2(bc).

By Lemma 2.1(b), $\nu(\lambda)$ is the largest partition in $P_{0}(n)$ with Ferrers diagram contained in $D(\lambda)$. So we need only show that $\lambda-\nu$ is a vertical strip. Write $\lambda=\left(\alpha_{1}, \ldots, \alpha_{p} \mid \beta_{1}, \ldots, \beta_{p}\right)$ and suppose $\lambda_{i}-\nu_{i}>1$. We have $\left(i, \lambda_{i}-1\right) \notin D(\nu)$, which means that $\lambda_{i}+\lambda_{\lambda_{i}-1}<i+\lambda_{i}-1$. Subtracting $\lambda_{i}$ from both sides, we have $\lambda_{\lambda_{i}-1}<i-1$; equivalently, $\lambda_{i-1}^{\prime}<\lambda_{i}-1$. If $i \leq p$, then $\lambda_{i-1}^{\prime}=\beta_{i-1}+i-1$ and $\lambda_{i}=\alpha_{i}+i$. Therefore $\alpha_{i}>\beta_{i-1}$, and we conclude that $\alpha_{i-1} \geq \alpha_{i}+1>\beta_{i-1}+1$, which contradicts the assumption that $\lambda \in P_{0,1}(n)$. Suppose instead that $i>p$. Then $\lambda_{i} \leq p$, so $\lambda_{\lambda_{i}-1}=\alpha_{\lambda_{i}-1}+\lambda_{i}-1$. Meanwhile, $\lambda_{\lambda_{i}}^{\prime} \geq i$ for any $\lambda$, and since $i>p \geq \lambda_{i}$, we can write $\lambda_{\lambda_{i}}^{\prime}=\beta_{\lambda_{i}}+\lambda_{i}$. We see that

$$
\beta_{\lambda_{i}}+1 \geq i-\lambda_{i}+1>i-\lambda_{i}>\lambda_{\lambda_{i-1}}-\lambda_{i}+1=\alpha_{\lambda_{i}-1},
$$

which again implies $\lambda \notin P_{0,1}(n)$. We have proved that $\lambda_{i}-\nu_{i} \leq 1$ for all $i \in[n]$ whenever $\lambda \in P_{0,1}(n)$.

Observe that $\bar{\tau}$, the order ideal of $\left\langle\begin{array}{c}{[n]} \\ 2\end{array}\right\rangle$ corresponding to $\nu$, is $\{(i, j) \in D(\nu)$ : $i \leq j\}$. If $(i, j) \in Z\left(\bar{\tau}, \omega_{ \pm}\right)$, then $i<j$ and we have $\nu_{i}=\#_{0}(i, \bar{\tau})=j$ and 
$\nu_{j}=\#_{0}(j, \bar{\tau})=i$. And since $i, j \notin \omega_{ \pm}$, we have $\lambda_{i}=\nu_{i}$ and $\lambda_{j}=\nu_{j}$; therefore $\lambda_{i}+\lambda_{j}=i+j$. Meanwhile, if $i<j$ and $\lambda_{i}+\lambda_{j}=i+j$, then $(i, j) \in \bar{\tau}$. This implies that $\nu_{i} \geq j$ and $\nu_{j} \geq i$, so we must have $\nu_{i}=j$ (which implies $\left.(i, j+1) \notin \bar{\tau}\right) ; \nu_{j}=i$ (meaning $(i+1, j) \notin \bar{\tau})$; and $i, j \notin \omega_{ \pm}$. We conclude $(i, j) \in Z\left(\bar{\tau}, \omega_{ \pm}\right)$, and we have shown that $\left|Z\left(\bar{\tau}, \omega_{ \pm}\right)\right|=z(\lambda)$.

\section{PRoOf OF LEMMA $7.2(\mathrm{~cd})$}

This proof differs somewhat from the previous two. Lemma 2.3 tells us that for any $\lambda \in P_{-1,1}(n)$, there is a unique largest partition $\kappa=\kappa(\lambda)$ among those partitions $\eta \in P_{-1}(n)$ for which $\lambda-\eta$ is a disjoint union of dominos with no more than one domino per row of $D(\lambda)$. Now given $\lambda \in P_{-1,1}(n)$, let $\tau$ be the order ideal of $\left(\begin{array}{c}{[n]} \\ 2\end{array}\right)$ corresponding to $\kappa(\lambda)$. Let $\omega_{0}=\left\{i \in[n]: \lambda_{i}-\kappa_{i}=2\right\}$ and $\omega_{ \pm}=\left\{i \in[n]: \lambda_{i}-\kappa_{i}=1\right.$ or 2$\}$. Observe that $q(\lambda)=\left|\omega_{0}\right|$. For each $\sigma \in S_{n}$, there exists $\mathfrak{g} \in \mathfrak{C}_{n}^{* * *}$ such that: $o\left(\sigma(i), \mathfrak{g}^{+}\right)=n-i$ and $o(\sigma(i), \mathfrak{g})=\lambda_{i}+n-i$ for each $i \in[n] ; \tau=\tau(\mathfrak{g}) ; \omega_{0}=\omega_{0}(\mathfrak{g})$; and $\omega_{ \pm}=\omega_{ \pm}(\mathfrak{g})$. For example, if $\lambda=(3,3,2)$, then $\kappa=(2,2,2), \tau=\{(1,2),(1,3),(2,3)\}, \omega_{0}$ is empty, and $\omega_{ \pm}=\{1,2\}$. Figure 7.3 shows $\mathfrak{g} \in \mathfrak{C}_{3}$ corresponding to this $\lambda$ and to the identity permutation.

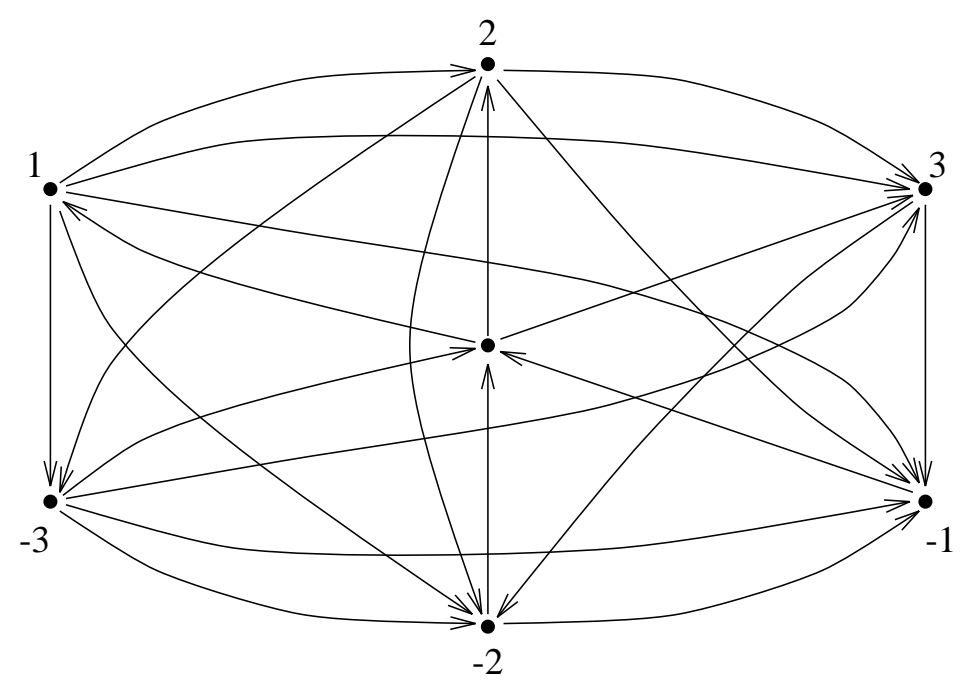

Figure 7.3

When $\mathfrak{g}$ is defined as in the preceding paragraph, we have

$$
\breve{w}(\mathfrak{g})=(-1)^{\sigma} x^{\sigma\left(\lambda+\delta_{n}\right)}(-1)^{|\kappa| / 2+q(\lambda)} t^{(|\lambda|-|\kappa|) / 2} .
$$

Fix $\lambda \in P_{-1,1}(n)$ and $\sigma \in S_{n}$ and let $\mathfrak{g} \in \mathfrak{C}_{n}^{* *}$ be as in the preceding paragraph. If $\mathfrak{g}^{*}$ is any digraph in $\mathfrak{C}_{n}^{* *}$ with $\left(\mathfrak{g}^{*}\right)^{+}=\mathfrak{g}^{+}$and $o\left(\sigma(i), \mathfrak{g}^{*}\right)=o(\sigma(i), \mathfrak{g})$ for each $i \in[n]$, then $\tau\left(\mathfrak{g}^{*}\right) \subseteq \tau, \omega_{0}\left(\mathfrak{g}^{*}\right) \supseteq \omega_{0}$, and $\omega_{ \pm}\left(\mathfrak{g}^{*}\right) \supseteq \omega_{ \pm}$. Let $Z(\lambda)=\{(i, j): i<$ $\left.j, \lambda_{i}+\lambda_{j}=i+j\right\}$, so that $|Z(\lambda)|=z(\lambda)$. Now it suffices to prove the following: 
(i) For every subset $A$ of $Z(\lambda)$, there is a digraph $\mathfrak{g}_{A} \in \mathfrak{C}_{n}^{* *}$ such that: $\mathfrak{g}_{A}^{+}=\mathfrak{g}^{+}$; $o\left(\sigma(i), \mathfrak{g}_{A}\right)=o(\sigma(i), \mathfrak{g})$ for each $i \in[n]$; and $\breve{w}\left(\mathfrak{g}_{A}\right)=\left(-t^{2}\right)^{|A|} \breve{w}(\mathfrak{g})$.

(ii) If $\lambda_{i}=i$ for some $i \in[n]$, then for each $\mathfrak{g}_{A}$ there is a $\mathfrak{g}_{A}^{\prime} \in \mathfrak{C}_{n}^{* * *}$ with the same positive subtournament and out-degrees as $\mathfrak{g}_{A}$ and with $\breve{w}\left(\mathfrak{g}_{A}^{\prime}\right)=-t \breve{w}\left(\mathfrak{g}_{A}\right)$.

(iii) Every $\mathfrak{g}^{*} \in \mathfrak{C}_{n}^{* *}$ having the same positive subtournament and out-degrees as $\mathfrak{g}$ is of the form $\mathfrak{g}_{A}$ or $\mathfrak{g}_{A}^{\prime}$ for some $A \subseteq Z(\lambda)$.

To prove (i), suppose first of all that $A=\{(i, j)\}$. Since $0 \leq \lambda_{k}-\kappa_{k} \leq 2$ for all $k \in[n]$, we must have $i+j-4 \leq \kappa_{i}+\kappa_{j} \leq i+j$. Observe that $\kappa_{i}+\kappa_{j}$ is at most $i+j-3$ if $(i, j) \notin \tau$ and at least $i+j-1$ if $(i, j) \in \tau$, so we can rule out $\kappa_{i}+\kappa_{j}=i+j-2$. The remaining possibilities are as follows:

$\kappa_{i}+\kappa_{j}=i+j$. We have $(i, j) \in \tau$, and there is exactly one $k$ such that either $k>j$ and $(i, k) \in \tau$ or $k>i$ and $(k, j) \in \tau$. Either $k=i+1$ and $(i+1, j) \in I(\tau)$, or $k=j+1$ and $(i, j+1) \in I(\tau)$. We have $i \notin \omega_{0}, i \notin \omega_{ \pm}, j \notin \omega_{0}$, and $j \notin \omega_{ \pm}$. Suppose $(i+1, j) \in I(\tau)$. We find that $i$ and $i+1$ are interchangeable in $\tau$, so $\kappa_{i+1}=\kappa_{i}=\lambda_{i} \geq \lambda_{i+1}$; this means that $i+1$ cannot be in $\omega_{0}$ or $\omega_{ \pm}$. We define $\mathfrak{g}_{A}$ by putting $\tau\left(\mathfrak{g}_{A}\right)=\tau \backslash\{(i, j),(i+1, j)\} ; \omega_{0}\left(\mathfrak{g}_{A}\right)=\omega_{0} \cup\{j\}$; and $\omega_{ \pm}\left(\mathfrak{g}_{A}\right)=\omega_{ \pm} \cup\{i, i+1, j\}$. A similar argument applies if $(i, j+1) \in I(\tau)$ : we have $\tau\left(\mathfrak{g}_{A}\right)=\tau \backslash\{(i, j),(i, j+1)\}, \omega_{0}\left(\mathfrak{g}_{A}\right)=\omega_{0} \cup\{i\}$, and $\omega_{ \pm}\left(\mathfrak{g}_{A}\right)=\omega_{ \pm} \cup\{i, j, j+1\}$. Figure 7.4 gives an example of this construction in the situation $\lambda=(6,3,2,2,1)$, with $A=\{(2,3)\}$. On the left are $\tau, \omega_{0}$, and $\omega_{ \pm}$for $\mathfrak{g}$, and on the right, the corresponding sets for $\mathfrak{g}_{A}$. As in Figure 6.4 , each square $(k, k)$ is vertically lined if $k \in \omega_{0}$ and horizontally lined if $k \in \omega_{ \pm}$.

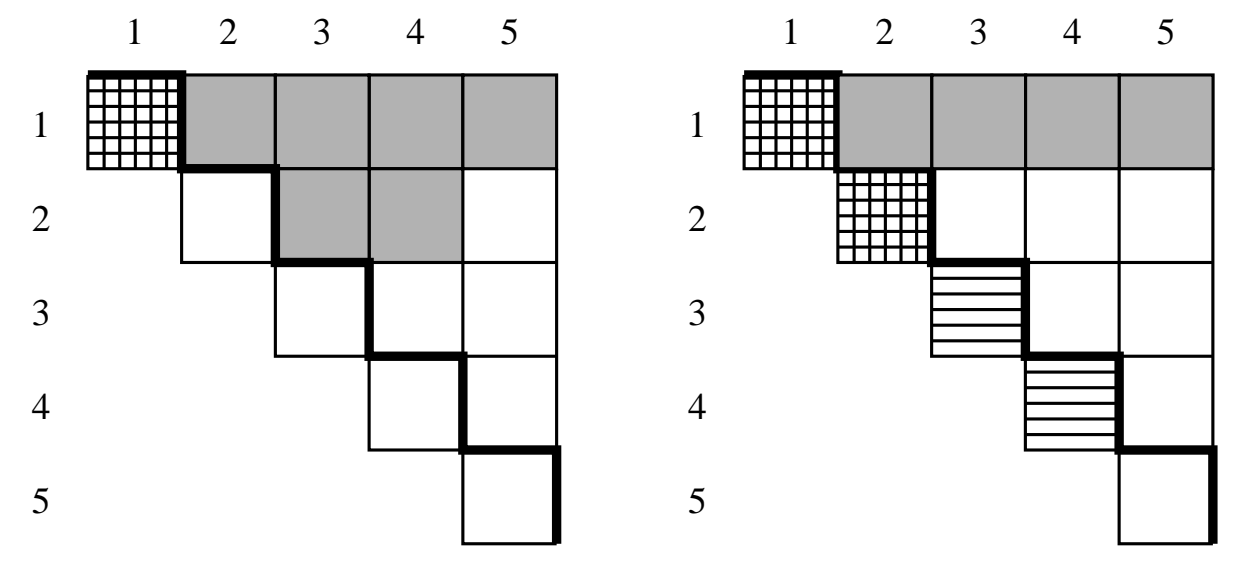

FIGURE 7.4

$\kappa_{i}+\kappa_{j}=i+j-1$. We have $(i, j) \in I(\tau)$; neither $i$ nor $j$ is in $\omega_{0}$ and exactly one of them is in $\omega_{ \pm}$. If $j<n$, then $j$ and $j+1$ are not interchangeable in $\tau$; if $j>i+1$, then $i$ and $i+1$ are not interchangeable in $\tau$. Suppose $j \in \omega_{ \pm}$. Then $j$ and $j-1$ must be interchangeable in $\tau, j-1 \in \omega_{ \pm}$, and $j-1 \notin \omega_{0}$ : otherwise, $\lambda-\kappa$ would not be a disjoint union of dominos with at most one domino per row. If $j-1=i$, then we would have $\lambda_{i}+\lambda_{j}=i+j+1$, so we must have $j>i+1$. Alternatively, suppose $i \in \omega_{ \pm}$. Then $i-1$ or $i+1$ must be in $\omega_{ \pm}$and not in $\omega_{0}$; 
$i$ and $i+1$ are not interchangeable in $\tau$ unless $i+1=j$, but $j \notin \omega_{ \pm}$; we conclude that $i$ and $i-1$ are interchangeable in $\tau, i-1 \in \omega_{ \pm}$, and $i-1 \notin \omega_{0}$. If $j \in \omega_{ \pm}$, then let $\tau\left(\mathfrak{g}_{A}\right)=\tau \backslash\{(i, j-1),(i, j)\} ; \omega_{0}\left(\mathfrak{g}_{A}\right)=\omega_{0} \cup\{i, j-1, j\}$; and $\omega_{ \pm}\left(\mathfrak{g}_{A}\right)=\omega_{ \pm} \cup\{i\}$. If $i \in \omega_{ \pm}$, then $\tau\left(\mathfrak{g}_{A}\right)$ is $\tau \backslash\{(i-1, j),(i, j)\}, \omega_{0}\left(\mathfrak{g}_{A}\right)=\omega_{0} \cup\{i-1, i, j\}$, and $\omega_{ \pm}\left(\mathfrak{g}_{A}\right)=\omega_{ \pm} \cup\{j\}$. In Figure 7.5 , we have an example of this construction for $\lambda=(4,3,3,2,2)$ and $A=\{(1,5)\}$.

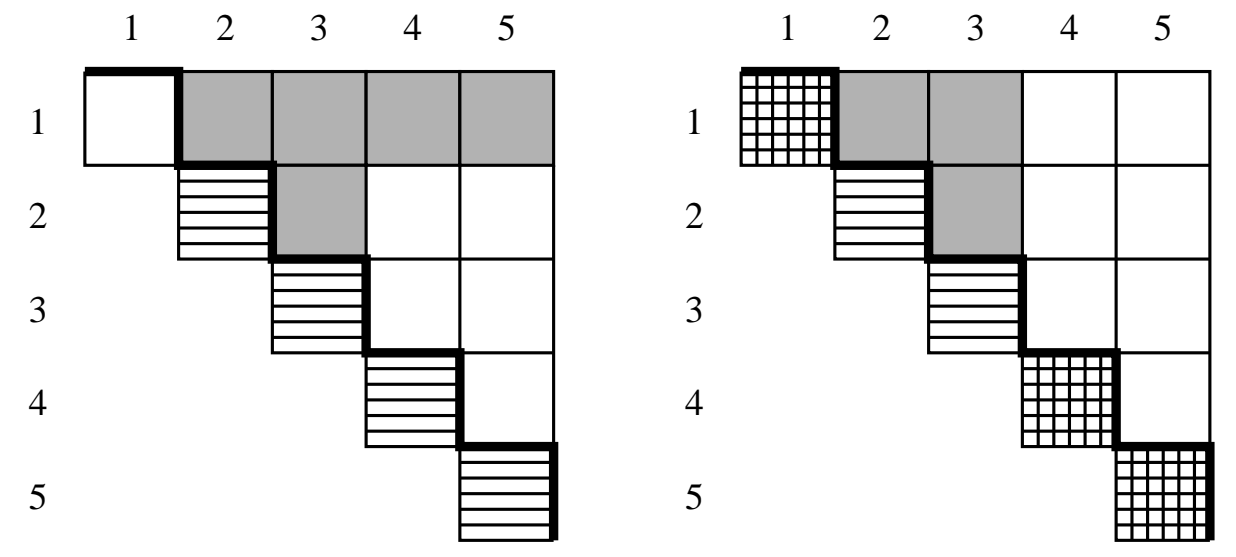

FIgURE 7.5

$\kappa_{i}+\kappa_{j}=i+j-3$. We have $(i, j) \in O(\tau)$ and one of $i$ and $j$ is in both $\omega_{0}$ and $\omega_{ \pm}$, while the other is in $\omega_{ \pm}$only. If $j \notin \omega_{0}$, then we must have $j$ and $j+1$ interchangeable in $\tau, j+1 \in \omega_{ \pm}$, and $j+1 \notin \omega_{0}$. This is because $j$ has to be "paired off" with either $j-1$ or $j+1$ according to the definition of $\mathfrak{C}_{n}^{* *}$, and assuming it is paired off with $j-1$ leads to a contradiction. But in this case, we can replace $\tau$ with $\tau \cup\{(i, j),(i, j+1)\}$, remove $i, j$, and $j+1$ from $\omega_{ \pm}$, and remove $i$ from $\omega_{0}$; this contradicts our assumption that $\tau$ corresponds to $\kappa$. We also derive this contradiction if $i \notin \omega_{0}$. We find that if $i>1$, then $i$ and $i-1$ are not interchangeable in $\tau, i$ is paired off with $i+1$, and $j>i+1$; we can replace $\tau$ with $\tau \cup\{(i, j),(i+1, j)\}$.

$\kappa_{i}+\kappa_{j}=i+j-4$. Here too we have a contradiction of the assumption that $\tau$ corresponds to $\kappa$. We can add either $\{(i, j-1),(i, j)\}$ or $\{(i-1, j),(i, j)\}$ to $\tau$ and change $\omega_{0}$ and $\omega_{ \pm}$accordingly. For instance, one possibility is $(i, j-1) \in O(\tau)$, with $j-1$ and $j$ interchangeable in $\tau$. We have both $i$ and $j$ in $\omega_{0}$ and $\omega_{ \pm} ; j-1$ is also in $\omega_{0}$ and $\omega_{ \pm}$, as otherwise we would have $\lambda_{j-1}<\lambda_{j}$. We can add $\{(i, j-1),(i, j)\}$ to $\tau$.

We have proved (i) whenever $|A|=1$. In this case, we obtain $\tau\left(\mathfrak{g}_{A}\right)$ by removing from $\tau$ a set of the form $\{(i, j),(i+1, j)\}$ or $\{(i, j),(i, j+1)\}$. Such a set is a domino. In case $|A|>1$, we can find a domino to remove from $\tau$ for each $(i, j) \in A$. If no two of these dominos intersect, then we can construct $\mathfrak{g}_{A}$ "one domino at a time" by repeating the above construction for one-element sets $A$. (This is essentially what we did in proving the first two parts of Lemma 7.2 , only with monominos instead of dominos.) On the other hand, it is possible for two dominos to intersect. 
For instance, this happens if $\lambda=(3,3,2,2)$ and $A=\{(1,4),(2,3)\}$ : the dominos corresponding to $(1,4)$ and $(2,3)$ are respectively $\{(1,4),(2,4)\}$ and $\{(2,3),(2,4)\}$.

We claim, however, that given any three dominos that correspond to elements of $Z(\lambda)$, one of them is disjoint from the other two. This is because $i \neq k$ and $j \neq l$ whenever $(i, j)$ and $(k, l)$ are distinct elements of $Z(\lambda)$. The only way two dominos can intersect is if they are of the form $\{(i, j-1),(i, j)\}$ and $\{(i-1, j),(i, j)\}$, with $(i, j-1),(i-1, j) \in Z(\lambda)$. It is not hard to see that no third domino corresponding to an element of $Z(\lambda)$ can intersect either of these. So to complete the proof of (i), we need only show that if $A=\{(i, j-1),(i-1, j)\}$, with corresponding dominos $\{(i, j-1),(i, j)\}$ and $\{(i-1, j),(i, j)\}$, then we can create $\mathfrak{g}_{A} \in \mathfrak{C}_{n}^{* *}$ having the same positive subtournament and out-degrees as $\mathfrak{g}$ and with $\breve{w}\left(\mathfrak{g}_{A}\right)=t^{4} \breve{w}(\mathfrak{g})$. For such an $A$, we have: none of $i-1, i, j-1, j$ is in $\omega_{0}$ or $\omega_{ \pm} ; i-1$ and $i$ are interchangeable in $\tau ; j-1$ and $j$ are interchangeable in $\tau$; and $(i, j) \in I(\tau)$. We define

$$
\begin{aligned}
\tau\left(\mathfrak{g}_{A}\right) & =\tau \backslash\{(i-1, j-1),(i-1, j),(i, j-1),(i, j)\} \\
\omega_{0}\left(\mathfrak{g}_{A}\right) & =\omega_{0} \cup\{i-1, i, j-1, j\} \\
\omega_{ \pm}\left(\mathfrak{g}_{A}\right) & =\omega_{ \pm} \cup\{i-1, i, j-1, j\} .
\end{aligned}
$$

We think of $\tau\left(\mathfrak{g}_{A}\right)$ as being obtained from $\tau$ by removing two dominos. This completes the proof of claim (i).

We now begin the proof of (ii). Let $\mathfrak{g}_{A} \in \mathfrak{C}_{n}^{* * *}$ be the digraph corresponding to some $A \subseteq Z(\lambda)$, where $\lambda$ is such that $\lambda_{i}=i$ for some $i \in[n]$. Recall that $\tau$ denotes the order ideal of $\left(\begin{array}{c}{[n]} \\ 2\end{array}\right)$ corresponding to $\kappa=\kappa(\lambda)$ and that $\omega_{0}=\{i \in[n]$ : $\left.\lambda_{i}-\kappa_{i}=2\right\}$ and $\omega_{ \pm}=\left\{i \in[n]: \lambda_{i}-\kappa_{i}=1\right.$ or 2$\}$. Let $\tau_{A}=\tau\left(\mathfrak{g}_{A}\right)$. We claim that either $(i, i+1) \in I\left(\tau_{A}\right)$ or $(i-1, i) \in I\left(\tau_{A}\right)$. There are three possible values for $\kappa_{i}: i, i-1$, and $i-2$. If $\kappa_{i}=i$, then $(i, i+1) \in I(\tau)$. If $\kappa_{i}=i-1$, then $(i-1, i) \in \tau$ and $(i, i+1) \notin \tau$. We have $i \in \omega_{ \pm}$and $i \notin \omega_{0}$. Assuming that $(i-1, i+1) \in \tau$, we conclude that $i-1$ and $i$ are not interchangeable in $\tau$, so $i$ and $i+1$ must be, and we must have $i+1 \in \omega_{ \pm}$and $i+1 \notin \omega_{0}$. But this contradicts the maximality of $\tau$, since we can add $(i, i+1)$ to it and remove $i$ and $i+1$ from $\omega_{ \pm}$. So we must have $(i-1, i+1) \notin \tau$, and therefore $(i-1, i) \in I(\tau)$. Similarly, if $\kappa_{i}=i-2$, we find that we can add $(i-1, i)$ to $\tau$ and remove $i-1$ and $i$ from $\omega_{0}$. We have proved the claim for $\tau_{A}=\tau$ (this is the case $A=\emptyset$ ). If $A$ is not empty, then we obtain $\tau_{A}$ by removing dominos from $\tau$. If $(i, i+1)$ is in $\tau_{A}$, then it is in $I\left(\tau_{A}\right)$. If $(i, i+1)$ is in $I(\tau)$ but not $\tau_{A}$, then it must be contained in a domino removed from $\tau$; the other member of this domino must be $(i-1, i+1)$. Having removed this domino, we have $(i-1, i) \in I\left(\tau_{A}\right)$, unless $(i-1, i)$ is also in a domino removed from $\tau$, which it cannot be, since the other member of the domino would be $(i-2, i)$; removing both dominos would reduce $\#(i, \tau)$ by 3 , and we could not have $o\left(\sigma(i), \mathfrak{g}_{A}\right)=o(\sigma(i), \mathfrak{g})$. A similar argument proves that if $(i-1, i) \in I(\tau)$, then we must have $(i-1, i) \in I\left(\tau_{A}\right)$. We have proved that $(i, i+1)$ or $(i-1, i)$ is in $I\left(\tau_{A}\right)$ for any $A \subseteq Z(\lambda)$.

Now we know that either $(i, i+1) \in I\left(\tau_{A}\right), i \notin \omega_{0}\left(\mathfrak{g}_{A}\right)$, and $i \notin \omega_{ \pm}\left(\mathfrak{g}_{A}\right)$; or $(i-1, i) \in I\left(\tau_{A}\right), i \in \omega_{ \pm}\left(\mathfrak{g}_{A}\right)$, and $i \notin \omega_{0}\left(\mathfrak{g}_{A}\right)$. In the former case, we see that $i$ and 
$i+1$ are interchangeable in $\tau$ and that $i+1$ is not in $\omega_{0}\left(\mathfrak{g}_{A}\right)$ or $\omega_{ \pm}\left(\mathfrak{g}_{A}\right)$ : otherwise we would have $o\left(\sigma(i), \mathfrak{g}_{A}\right) \leq o\left(\sigma(i+1), \mathfrak{g}_{A}\right)$. So we create $\mathfrak{g}_{A}^{\prime}$ by putting $\tau\left(\mathfrak{g}_{A}^{\prime}\right)=$ $\tau\left(\mathfrak{g}_{A}\right) \backslash\{(i, i+1)\} ; \omega_{0}\left(\mathfrak{g}_{A}^{\prime}\right)=\omega_{0}\left(\mathfrak{g}_{A}\right)$; and $\omega_{ \pm}\left(\mathfrak{g}_{A}^{\prime}\right)=\omega_{ \pm}\left(\mathfrak{g}_{A}\right) \cup\{i, i+1\}$. In the latter case, we must have $i-1 \in \omega_{ \pm}\left(\mathfrak{g}_{A}\right)$ and $i-1 \notin \omega_{0}\left(\mathfrak{g}_{A}\right)$, since $i$ and $i+1$ are not interchangeable in $\tau\left(\mathfrak{g}_{A}\right)$. We construct $\mathfrak{g}_{A}^{\prime}$ by putting $\tau\left(\mathfrak{g}_{A}^{\prime}\right)=\tau\left(\mathfrak{g}_{A}\right) \backslash\{(i-1, i)\}$; $\omega_{0}\left(\mathfrak{g}_{A}^{\prime}\right)=\omega_{0}\left(\mathfrak{g}_{A}\right) \cup\{i-1, i\}$; and $\omega_{ \pm}\left(\mathfrak{g}_{A}^{\prime}\right)=\omega_{ \pm}\left(\mathfrak{g}_{A}\right)$. This completes the proof of (ii).

We shall prove (iii) by constructing a subset $A$ of $Z(\lambda)$ for each $\mathfrak{g}^{*} \in \mathfrak{C}_{n}$ having the same positive subtournament and out-degrees as $\mathfrak{g}$. Recall the definitions of $\tau$, $\omega_{0}$, and $\omega_{ \pm}$relative to $\sigma \in S_{n}$ and $\lambda \in P_{-1,1}(n)$; in particular, $\tau$ is the order ideal of $\left(\begin{array}{c}{[n]} \\ 2\end{array}\right)$ corresponding to $\kappa(\lambda)$. Let $\tau^{*}=\tau\left(\mathfrak{g}^{*}\right), \omega_{0}^{*}=\omega_{0}\left(\mathfrak{g}^{*}\right)$, and $\omega_{ \pm}^{*}=\omega_{ \pm}\left(\mathfrak{g}^{*}\right)$. Observe that if $\tau^{*} \neq \tau$, then there exists $(i, j) \in I(\tau) \backslash \tau^{*}$. This is because $\tau$ and $\tau^{*}$ are order ideals and we obtain $\tau^{*}$ from $\tau$ by removing some elements. Now to construct $A$, we begin by setting $A=\emptyset$ and do the following for each $(i, j) \in I(\tau) \backslash \tau^{*}:$

When $j=i+1$, we distinguish between two cases: $(i-1, i+1) \in \tau^{*}$ and $(i-1, i+1) \in \tau \backslash \tau^{*}$. In the first case, we find that $\#(i, \tau)=\#(i+1, \tau)=i$ and that $\#\left(i, \tau^{*}\right)=\#\left(i+1, \tau^{*}\right)=i-1$. We have either $i, i+1 \in \omega_{ \pm} \backslash \omega_{0}$, meaning $\lambda_{i+1}=i+1$, or $i, i+1 \notin \omega_{ \pm}$, meaning $\lambda_{i}=i$. We do not add anything to $A$, but we see that $\mathfrak{g}^{*}$ is of the form $\mathfrak{g}_{A}^{\prime}$ rather than $\mathfrak{g}_{A}$. In the second case, $\#(i+1, \tau)=i$ and $\#\left(i+1, \tau^{*}\right)=i-2$; we must have $i+1 \notin \omega_{ \pm}$and $i+1 \in \omega_{0}^{*}$. So $\lambda_{i+1}=i$ in this case. Meanwhile, $\#\left(i-1, \tau^{*}\right)=\#(i-1, \tau)-1$ and $\#\left(i, \tau^{*}\right)=\#(i, \tau)-1$; this means $i-1$ and $i$ are "paired" as members of $\omega_{ \pm} \backslash \omega_{0}$ or of $\omega_{ \pm}^{*} \backslash \omega_{0}^{*}$, so they must be interchangeable in $\tau$. Since $\#(i, \tau)=i$, we must have $\#(i-1, \tau)=i$. If $i-1$ and $i$ are in $\omega_{ \pm} \backslash \omega_{0}$, then $\lambda_{i}=i+1$; we add $(i, i+1)$ to $A$. Otherwise, $i-1$ and $i$ are not in $\omega_{ \pm}$and $\lambda_{i-1}=i$; we add $(i-1, i+1)$ to $A$. In the latter situation, we may also have $\mathfrak{g}^{*}$ of the form $\mathfrak{g}_{A}^{\prime}$. This happens if $(i-1, i) \in \tau \backslash \tau^{*}$, in which case $\#\left(i, \tau^{*}\right)=\#(i, \tau)-2$, so $i$ cannot be in $\omega_{ \pm}$.

If $j>i+1$, then it is impossible to remove $(i, j)$ from $\tau$ and not remove either $(i-1, j)$ or $(i, j-1)$. The removal of only $(i, j)$ would force a violation of the pairing condition for members of $\omega_{ \pm} \backslash \omega_{0}$. So $(i-1, j) \in \tau \backslash \tau^{*}$ or $(i, j-1) \in \tau \backslash \tau^{*}$. If $(i-1, j) \in \tau \backslash \tau^{*}$ but $(i, j-1) \in \tau^{*}$, then we have $j \notin \omega_{ \pm}$, so $\lambda_{j}=\#(j, \tau)=i$. Meanwhile, $i-1$ and $i$ are interchangeable in $\tau$ : we have $\#\left(i-1, \tau^{*}\right)=\#(i-1, \tau)-1$ and $\#\left(i, \tau^{*}\right)=\#(i, \tau)-1$, so $i$ is paired with either $i-1$ or $i+1$ as a member of $\omega_{ \pm} \backslash \omega_{0}$ or of $\omega_{ \pm}^{*} \backslash \omega_{0}^{*}$; but $i$ and $i+1$ are not interchangeable in $\tau$. We have $\#(i-1, \tau)=\#(i, \tau)=j-1$. If $i-1$ and $i$ are in $\omega_{ \pm}$, then $\lambda_{i-1}=\lambda_{i}=j$, and we add $(i, j)$ to $A$. Otherwise, $\lambda_{i-1}=\lambda_{i}=j-1$, and we add $(i-1, j)$ to $A$. Similarly if $(i, j-1) \in \tau \backslash \tau^{*}$, but $i=1$ or $(i-1, j) \in \tau^{*}$ : we add either $(i, j-1)$ or $(i, j)$ to $A$. Finally, it is possible that both $(i, j-1)$ and $(i-1, j)$ are in $\tau \backslash \tau^{*}$. In this case, we must also have $(i-1, j-1) \in \tau \backslash \tau^{*}$. To see this, suppose $(i-1, j-1) \in \tau^{*}$. We find that $\#\left(i, \tau^{*}\right)=\#(i, \tau)-2$ but $\#\left(i-1, \tau^{*}\right)=\#(i-1, \tau)-1$; if $i=2$, we have a violation of the pairing condition for $\omega_{ \pm} \backslash \omega_{0}$; otherwise, $i-1$ must be paired with $i-2$. This in turn forces $(i-2, j) \in \tau \backslash \tau^{*}$, which gives us $\#\left(j, \tau^{*}\right) \leq \#(j, \tau)-3$, which means that $\sigma(j)$ couldn't have the same out-degree in $\mathfrak{g}^{*}$ as in $\mathfrak{g}$. So we have 
$(i-1, j-1) \in \tau \backslash \tau^{*}$, and none of $i-1, i, j-1, j$ is in $\omega_{0}$ or $\omega_{ \pm}$. We find that $\lambda_{i-1}=\lambda_{i}=j$ and $\lambda_{j-1}=\lambda_{j}=i-1$, and add $(i-1, j)$ and $(i, j-1)$ to $A$. This concludes our description of the construction of $A$, and thus completes the proof of Lemma $7.2(\mathrm{~cd})$.

We conclude this section with an example of the construction described above. Fix $\sigma \in S_{6}$ and let $\mathfrak{g} \in \mathfrak{C}_{6}^{* * *}$ be the digraph whose positive subtournament corresponds to $\sigma$ and for which $\tau, \omega_{0}$, and $\omega_{ \pm}$are as in Figure 7.6 .

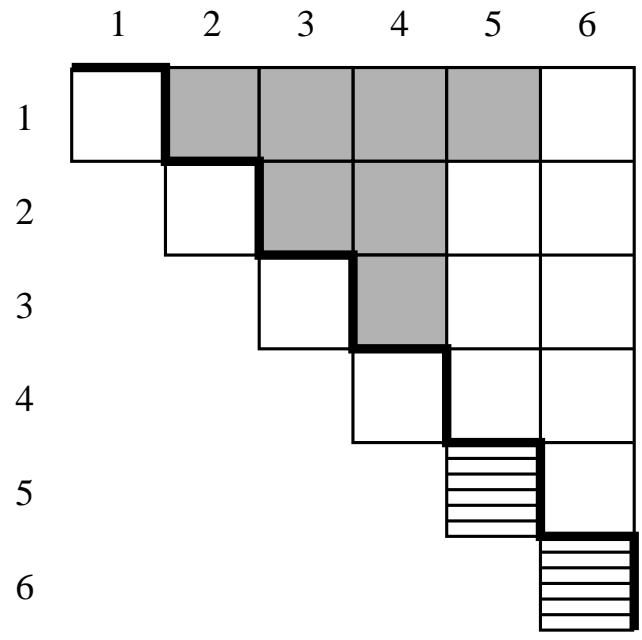

Figure 7.6

Now $\mathfrak{g}$ corresponds to the partition $\lambda=(5,3,3,3,2,2)$, and $\tau(\mathfrak{g})$ is the order ideal of $\left(\begin{array}{c}{[n]} \\ 2\end{array}\right)$ corresponding to $\kappa(\lambda)$. Another digraph $\mathfrak{g}^{*}$ with the same positive subtournament and out-degrees as $\mathfrak{g}$ is given by $\tau^{*}, \omega_{0}^{\prime}$, and $\omega_{ \pm}^{\prime}$ as depicted in Figure 7.7 .

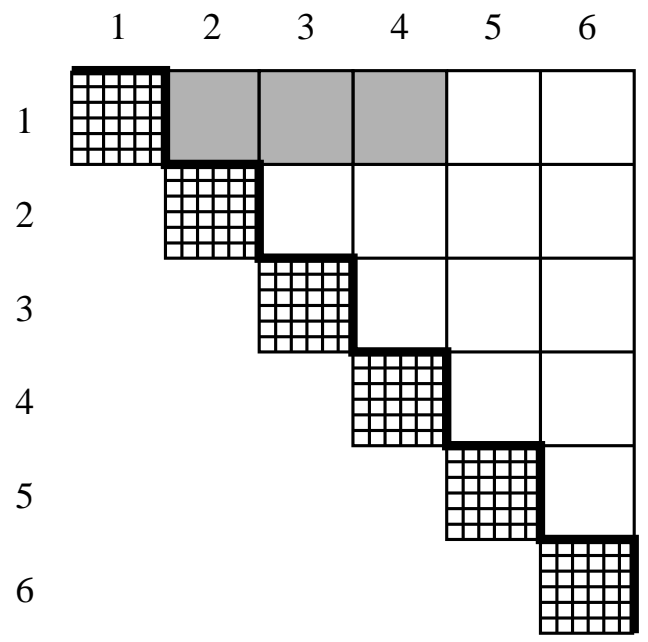

FiguRE 7.7 
We see that $I(\tau) \backslash \tau^{*}=\{(1,6),(3,4)\}$. Begin with $A=\emptyset$ and $(i, j)=(1,6)$; $i=1$ and $j>i+1$, so we must have $(1,5) \in \tau \backslash \tau^{*}$. Observe that 5 and 6 are interchangeable in $\tau$, and are both in $\omega_{ \pm}$. Hence we have $\lambda_{1}=\#(1, \tau)=5$ and $\lambda_{6}=\#(6, \tau)+1=2$; we add $(1,6)$ to $A$. Now we set $(i, j)=(3,4)$. We observe that $(2,4) \in \tau \backslash \tau^{*} ; 2$ and 3 are interchangeable in $\tau$, and are both not in $\omega_{ \pm}$. Therefore $\lambda_{2}=\#(2, \tau)=3$ and $\lambda_{4}=\#(4, \tau)=3$; we add $(2,4)$ to $A$. Finally, we see that $(2,3) \in \tau \backslash \tau^{*}$. Notice that the construction of $\mathfrak{g}_{A}$ from $\mathfrak{g}$ given in the proof of (i) would give us $\tau\left(\mathfrak{g}_{A}\right)=\tau \backslash\{(1,5),(1,6),(2,4),(3,4)\}$. Therefore $\tau^{*}=\tau\left(\mathfrak{g}_{A}\right) \backslash\{(2,3)\}$, and we have that $\mathfrak{g}^{*}$ is of the form $\mathfrak{g}_{A}^{\prime}$ rather than $\mathfrak{g}_{A}$.

\section{Appendix. Relation between Littlewood's identities and Weyl's formula}

The usual way of writing Weyl's denominator formula does not involve partitions, such as appear on the sum sides of $(2.3 \mathrm{~b}),(2.3 \mathrm{c})$, and $(2.3 \mathrm{~d})$. Instead, the terms on the sum side are indexed by elements of the Weyl group of the corresponding root system. We shall now show that $(2.3 \mathrm{c})$, which is equivalent to Littlewood's identity (2.2c), implies Weyl's formula for the root system $C_{n}$. Similar arguments may be used to show that (2.3b) and (2.3d) give Weyl's formula for $B_{n}$ and $D_{n}$ respectively. The following lemma is useful in each case.

Lemma A.1. ([S], Lemma 2.1.2) Let $t$ be an integer $\geq-1$. Then $\lambda \in P_{t}(n)$ if and only if $\ell(\lambda) \leq n$ and $\left\{\left|\lambda_{i}-i-\frac{t-1}{2}\right|: i \in[n]\right\}=\left\{\frac{t+1}{2}, \frac{\overline{t+3}}{2}, \ldots, \frac{t+2 n-1}{2}\right\}$.

In our proof of Lemma A.1, we shall use the following result, due to Macdonald:

Lemma A.2. ([M], Chapter I, (1.7)) For any partition $\lambda$ and positive integers $l \geq \ell(\lambda), m \geq \lambda_{1}$, the sets $\left\{\lambda_{i}+l-i: i \in[l]\right\}$ and $\left\{l-1+j-\lambda_{j}^{\prime}: j \in[m]\right\}$ are disjoint and their union is $\{0,1, \ldots, l+m-1\}$.

Proof of Lemma A.1. "If": Suppose $\ell(\lambda) \leq n$ and $\left\{\left|\lambda_{i}-i-\frac{t-1}{2}\right|: i \in[n]\right\}=\left\{\frac{t+1}{2}\right.$, $\left.\frac{t+3}{2}, \ldots, \frac{t+2 n-1}{2}\right\}$. Let $\left(\alpha_{1}, \ldots, \alpha_{p} \mid \beta_{1}, \ldots, \beta_{p}\right)$ be the Frobenius representation of $\lambda$. Then $\lambda_{i}-i-\frac{t-1}{2}=\alpha_{i}-\frac{t-1}{2}$ for each $i \in[p]$. Since the $\alpha_{i}$ are nonnegative, we cannot have $\alpha_{i}-\frac{t-1}{2} \leq-\frac{t+1}{2}$. So we must have $\alpha_{i}-\frac{t-1}{2} \geq \frac{t+1}{2}$ for all $i \in[p]$; furthermore, if $t=-1$, then $\alpha_{i}-\frac{t-1}{2}=\alpha_{i}+1>0=\frac{t+1}{2}$. On the other hand, $\lambda_{i}-i-\frac{t-1}{2}<-\frac{t-1}{2}$ for $p+1 \leq i \leq n$. We conclude that the positive elements of $\left\{\lambda_{i}-i-\frac{t-1}{2}: i \in[n]\right\}$ are $\alpha_{i}-\frac{t-1}{2}, i \in[p]$. Now choose $j \in[p]$ and let $b=\beta_{j}$. Since $b+j=\lambda_{j}^{\prime}$, we have $\lambda_{b+j} \geq j>\lambda_{b+j+1}$; hence $\lambda_{b+j}-b-j-\frac{t-1}{2}>-b-\frac{t+1}{2}>$ $\lambda_{b+j+1}-b-j-1-\frac{t-1}{2}$. What this means is that $b+\frac{t+1}{2} \notin\left\{\left|\lambda_{i}-i-\frac{t-1}{2}\right|: p+1 \leq\right.$ $i \leq n\}$. But the condition $\ell(\lambda) \leq n$ means that $0 \leq b \leq n-1$; so $b+\frac{t+1}{2} \in\left\{\frac{t+1}{2}\right.$, $\left.\frac{t+3}{2}, \ldots, \frac{t+2 n-1}{2}\right\}$. Therefore $b+\frac{t+1}{2}=\alpha_{i}-\frac{t-1}{2}$ for some $i ; \beta_{j}=\alpha_{i}+t$ for some $i$. Since this holds for each $j \in[n]$, and the sequences $\alpha$ and $\beta$ are both strictly decreasing, we conclude that $i=j$. Thus $\lambda \in P_{t}(n)$ as claimed.

"Only if": Let $\lambda=\left(\alpha_{1}+t, \ldots, \alpha_{p}+t \mid \alpha_{1}, \ldots, \alpha_{p}\right) \in P_{t}(n)$. Lemma A.2 tells us that $L=\left\{\lambda_{i}-i-\frac{t-1}{2}: i \in[n]\right\}$ and $L^{\prime}=\left\{j-\lambda_{j}^{\prime}-\frac{t+1}{2}: j \in[n+t]\right\}$ are disjoint, with $L \cup L^{\prime}=\left\{-n-\frac{t-1}{2},-n-\frac{t-1}{2}+1, \ldots, n+\frac{t-1}{2}\right\}$. We have 
$\lambda_{i}-i-\frac{t-1}{2}=\alpha_{i}+\frac{t+1}{2} \geq \frac{t+1}{2}$ if $i \in[p]$ and $\lambda_{i}-i-\frac{t-1}{2} \leq-\frac{t+1}{2}$ if $p+1 \leq i \leq n$. Therefore

$$
\left\{\left|\lambda_{i}-i-\frac{t-1}{2}\right|: i \in[n]\right\} \subseteq\left\{\frac{t+1}{2}, \frac{t+3}{2}, \ldots, \frac{t+2 n-1}{2}\right\} .
$$

Now to complete the proof, we need only show that there are $n$ distinct values in $\left\{\left|\lambda_{i}-i-\frac{t-1}{2}\right|: i \in[n]\right\}$, and to do this, it suffices to show that if $x>0$ and $x \in L$, then $-x \in L^{\prime}$. This is immediate: $x=\alpha_{i}+\frac{t+1}{2}$ for some $i \in[p]$, so $-x=-\alpha_{i}-\frac{t+1}{2}=i-\lambda_{i}^{\prime}-\frac{t+1}{2} \in L^{\prime}$.

Let $e_{i}$ denote the vector in $\mathbf{R}^{n}(n \geq i)$ having a 1 as its $i$ th component and 0 's elsewhere, so that $\left\{e_{1}, e_{2}, \ldots, e_{n}\right\}$ is the standard orthonormal basis of $\mathbf{R}^{n}$. The usual representation of $C_{n}$ relative to this basis is $\left\{ \pm\left(e_{i} \pm e_{j}\right): 1 \leq i<j \leq n, \pm 2 e_{i}\right.$ : $1 \leq i \leq n\}$, with positive subsystem $C_{n}^{+}=\left\{e_{i} \pm e_{j}: 1 \leq i<j \leq n, 2 e_{i}: 1 \leq i \leq n\right\}$. The Weyl group $W=W\left(C_{n}\right)$ acts on $C_{n}$ by permuting components and changing their signs. It is isomorphic to the semidirect product $Z_{2}^{n} \rtimes S_{n}$, where $Z_{2}$ is the cyclic group of order $2 ; Z_{2}^{n}$ is a normal subgroup of $W$ on which $S_{n}$ acts by automorphisms.

Now recall the usual statement of Weyl's denominator formula: Given a root system $\Phi$, with positive subsystem $\Phi^{+}$, in a Euclidean vector space $E$ with orthonormal basis $\left\{e_{1}, e_{2}, \ldots, e_{n}\right\}$, we have

$$
\prod_{\alpha \in \Phi^{+}}\left(x^{\alpha / 2}-x^{-\alpha / 2}\right)=\sum_{w \in W}(-1)^{w} x^{w(S)},
$$

where $x^{e_{i}}=x_{i}$ for each $i \in[n], W=W(\Phi)$ is the Weyl group of $\Phi$, and $S=$ $S\left(\Phi^{+}\right)=\frac{1}{2} \sum_{\alpha \in \Phi^{+}} \alpha$.

Multiplying both sides of $(2.3 \mathrm{c})$ by

$$
\prod_{i=1}^{n}\left(-x_{i}^{-1}\right) \prod_{1 \leq i<j \leq n}\left(-x_{i}^{-1 / 2} x_{j}^{-1 / 2}\right)\left(x_{i}^{-1 / 2} x_{j}^{-1 / 2}\right)=(-1)^{n(n+1) / 2} \prod_{i=1}^{n} x_{i}^{-n}
$$

we obtain

$$
\prod_{\alpha \in C_{n}^{+}}\left(x^{\alpha / 2}-x^{-\alpha / 2}\right)=\sum_{\substack{\lambda \in P_{1}(n) \\ \sigma \in S_{n}}}(-1)^{|\lambda| / 2+n(n+1) / 2}(-1)^{\sigma} \prod_{i=1}^{n} x_{\sigma(i)}^{\lambda_{i}-i}
$$

Meanwhile,

$$
S=\frac{1}{2} \sum_{\alpha \in C_{n}^{+}} \alpha=(n, \ldots, 2,1),
$$

and Lemma A.1 tells us that for $\lambda \in P_{1}(n)$, the sequence $\lambda^{-}=\left(\lambda_{1}-1, \ldots, \lambda_{n}-n\right)$ is obtained from $S$ by permutations and sign changes, i.e., that $\lambda^{-}=w(S)$ for some $w \in W$. Furthermore, since the sequences $\lambda^{-}$are all strictly decreasing, no two of them are in the same coset of $S_{n}$ in $W$. 
Given $\lambda \in P_{1}(n)$, let $w_{\lambda}$ be the unique $w \in W$ for which $\lambda^{-}=w(S)$. To prove that $(2.3 \mathrm{c})$ implies Weyl's formula for $C_{n}$, we must show that

$$
W / S_{n}=\left\{w_{\lambda}: \lambda \in P_{1}(n)\right\}
$$

is a complete set of coset representatives of $S_{n}$ in $W$, and that

$$
(-1)^{w_{\lambda}}=(-1)^{|\lambda| / 2+n(n+1) / 2}
$$

for each $\lambda \in P_{1}(n)$.

Let $\phi \in S_{n}$ and $s \in\{ \pm 1\}^{n}$ be such that $w_{\lambda}\left(e_{i}\right)=s_{i} e_{\phi(i)}$ for each vector $e_{i}$ in the standard orthonormal basis of $\mathbf{R}^{n}$. Then $\lambda_{i}-i=\left(n-\phi^{-1}(i)+1\right) s_{\phi^{-1}(i)}$ for each $i \in[n]$. So there must be some $p \in\{0,1, \ldots, n\}$ such that

$$
s_{\phi^{-1}}(i)= \begin{cases}1 & (1 \leq i \leq p) \\ -1 & (p+1 \leq i \leq n)\end{cases}
$$

and such that

$$
\phi^{-1}(1)<\phi^{-1}(2)<\cdots<\phi^{-1}(p) \quad \text { and } \quad \phi^{-1}(p+1)>\phi^{-1}(p+2)>\cdots>\phi^{-1}(n) .
$$

We have $p=p(\lambda)$, since $\lambda_{i}-i$ is positive for $i \leq p$ and negative for $i>p$. Observe that $s$ uniquely determines $\phi: \phi^{-1}(i)$ is either the $i$ th smallest $k$ such that $s_{k}=1$ or the $(i-p)$ th largest $k$ such that $s_{k}=-1$, according as $i \leq p$ or $i>p$. This means that the cardinality of $W / S_{n}$ is the cardinality of $\{ \pm 1\}^{n}$, which is the number of cosets of $S_{n}$ in $W$, as required. Meanwhile, the sign of $w_{\lambda}$ is $(-1)^{\phi} \prod_{i=1}^{n} s_{i}=(-1)^{\phi}(-1)^{n-p}$.

Choose $i, j \in[n]$ with $i<j$. Then $\phi(i)>\phi(j)$ if and only if $\phi(i)>p$. The number of inversions in $\phi$ is therefore $\sum_{i=p+1}^{n}\left(n-\phi^{-1}(i)\right)$, and the sign of $w_{\lambda}$ is -1 to the power

$$
\sum_{i=p+1}^{n}\left(n-\phi^{-1}(i)+1\right)=\sum_{i=p+1}^{n}\left(i-\lambda_{i}\right)
$$

Now observe that

$$
\begin{aligned}
\sum_{i=p+1}^{n}\left(i-\lambda_{i}\right) & =\sum_{i=p+1}^{n} i-\sum_{i=p+1}^{n} \lambda_{i} \\
& =\frac{n(n+1)}{2}-\frac{p(p+1)}{2}-|\lambda|+\sum_{i=1}^{p} \lambda_{i} \\
& =\frac{n(n+1)}{2}-|\lambda|+\sum_{i=1}^{p}\left(\lambda_{i}-i\right) ;
\end{aligned}
$$

since $|\lambda|$ is even and $\sum_{i=1}^{p}\left(\lambda_{i}-i\right)=|\lambda| / 2$ for any $\lambda \in P_{1}(n)$, we conclude that

$$
(-1)^{w_{\lambda}}=(-1)^{n(n+1) / 2-|\lambda| / 2}=(-1)^{n(n+1) / 2+|\lambda| / 2}
$$

as required. 


\section{Acknowledgment}

The author wishes to acknowledge the contributions of a referee, whose detailed suggestions helped to improve the clarity, style, and organization of this work.

\section{References}

[B] D. M. Bressoud, Colored tournaments and Weyl's denominator formula, Europ. J. Combinatorics 8 (1987), 245-255.

[BtD] T. H. Bröcker and T. tom Dieck, Representations of Compact Lie Groups, Springer-Verlag, New York, 1985.

[C] R. W. Carter, Simple Groups of Lie Type, Wiley, London, 1972.

[G] I. M. Gessel, Tournaments and Vandermonde's determinant, J. Graph Theory 3 (1979), $305-307$.

[H] J. E. Humphreys, Introduction to Lie Algebras and Representation Theory, Springer-Verlag, New York, 1972

[L] D. E. Littlewood, Theory of Group Characters and Matrix Representations of Groups, Clarendon, Oxford, 1940.

[M] I. G. Macdonald, Symmetric Functions and Hall Polynomials, Clarendon, Oxford, 1979.

[O] S. Okada, Alternating sign matrices and some deformations of Weyl's denominator formula, J. Alg. Combinatorics 2 (1993), 155-176.

[S] T. A. Simpson, Combinatorial Proofs and Generalizations of Weyl's Denominator Formula, Ph.D. Thesis, Penn State University, University Park, 1994.

[St] R. P. Stanley, Enumerative Combinatorics, volume I, Wadsworth \& Brooks/Cole, Monterey, 1986.

[W] H. Weyl, Theorie der Darstellung kontinuierlicher halbeinfacher Gruppen durch lineare Transformationen, Gesammelte Abhandlungen, Band II, Springer-Verlag, Heidelberg, 1968.

Department of Mathematics, Penn State University, University Park, PA 16802, USA

Current address: 7661 Tred Avon Circle, Easton, MD 21601, USA

E-mail address: todo@ora.nobis.com 